\title{
2022 ACMT Annual Scientific Meeting Abstracts - Virtual
}

\begin{abstract}
These are the abstracts of the 2022 American College of Medical Toxicology (ACMT) Annual Scientific Meeting (ASM). Due to the COVID-19 pandemic, this year's ASM is held in a virtual format. Included here are 147 abstracts accepted for presentation in March 2022, including research studies from around the world, new findings from the Toxicology Investigators Consortium (ToxIC), clinically significant case reports describing toxicologic phenomena, and noteworthy encore research from other scientific meetings. This year's ASM is a showcase of the innovative work medical toxicologists continue to do across the world despite challenges from COVID-19.
\end{abstract}

Keywords: Abstracts - Annual Scientific Meeting - Toxicology Investigators Consortium - Medical Toxicology Foundation

Corresponding Author: American College of Medical Toxicology (ACMT) 10645 N. Tatum Blvd Phoenix, AZ; info@acmt.net

Introduction: The American College of Medical Toxicology (ACMT) received 194 eligible research abstracts for consideration for presentation at the 2022 Annual Scientific Meeting (ASM), including 85 research studies and 109 case reports. Each abstract was reviewed in a blinded fashion by a minimum of three Abstract Review Committee members. Each abstract was independently scored based on the clinical question, data source, analytic method, results/conclusion, and clarity of presentation. The ACMT abstract chair and co-chair along with the Chair of the ACMT research committee then reviewed all abstracts and decided on their disposition. A total of 147 abstracts were accepted. Despite multiple waves of COVID-19 infection across the United States during the time abstract submissions were open, we received many high quality abstracts describing unique poisonings, the persistent epidemic of substance use disorders, and the intersection of medical toxicology and COVID-19. This year's ASM will once again be presented virtually due to the uncertainty around COVID-19, but the quality of submissions has continued to be exemplary and is a showcase of the innovative work medical toxicologists continue to do across the world despite challenges from COVID-19.

This work would not be possible without the hard work and diligence of our abstract reviewers: Justin Arnold, Mary Billington, Jeffrey Brent, Keith Burkhart, Vincent Calleo, Stephanie Carriero, Michael Chary, Richard Chen, James Alan Chenoweth, Neeraj Chhabra, Jon Cole, Nick Connors, Fiona Garlich, Rob Hendrickson, Michelle Hieger, David Juurlink, Louise Kao, Kenneth Katz, Ziad Kazzi, Joseph Kennedy, Andrew King, Shana Kusin, JoAn Laes, Eric Lavonas, Jacob Lebin, Michael Levine, David Liss, Alex Manini, Kevin Maskell, Christopher Meaden, William Joel Meggs, Andrew Monte, Elissa Moore, Babak Mostafazadeh, Mark Mycyk, Travis Olives, Anthony Pizon, Patricia Rosen, Jonathan Schimmel, Evan Schwarz, Samuel Stellpflug, Mark $\mathrm{Su}$, Manoj Tyagi, Shawn Varney, Steven Walsh, Richard Wang, Sage Weiner, Brandon Wills, and Luke Yip.

We would also like to thank Michael Chary, the chair of abstract review mentorship program, and the fellows-in-training who participated in the second year of the abstract review mentorship program: Marielle Alyssa Brenner, Chris Dion, Derek Fiske, Riley Hartmann, Michael Kennan, Brian Lewis, Gregory Makar, Brian Schultz, and Kyle Suen.
Equally significant is the contribution of Alison Meyn and Adrienne Dunavin from ACMT who led the process.

Congratulations to all the scholars whose work will be presented in March!

We look forward to a great virtual meeting and hope to see you all in person in 2023,

Peter R Chai, MD, FACEP, Abstract Review Chair Katherine L Boyle, MD, FACMT, Abstract Review Co-Chair Maryann Amirshahi, PharmD, MD, MPH, PhD, FACMT, Chair, ACMT Research Committee

\section{Platforms, Abstracts 001-008}

\section{Investigating MicroRNAs in Russell's Viper Bite Envenoming}

Shihana Fathima Salahudeen ${ }^{1,2}$, Anjana De Silva ${ }^{3}$, Mugdha V Joglekar ${ }^{4}$, Aanad A Hardika ${ }^{4}$, Devanshi Seth ${ }^{1,5}$, Geoffrey K Isbister, ${ }^{6,7}$, Nicholas A Buckley ${ }^{1,7}$

${ }^{1}$ The University of Sydney, Sydney, NSW, Australia. ${ }^{2}$ South Asian Clinical Toxicology of Research Collaboration, Peradeniya, NSW, Australia. ${ }^{3}$ Rajarata University of Sri Lanka, Mihintale, Sri Lanka. ${ }^{4}$ Western Sydney University, Campbelltown, NSW, Australia. ${ }^{5}$ Royal Prince Alfred Hospital, Camperdown, NSW, Australia. ${ }^{6}$ University of Newcastle, Newcastle, NSW, Australia. ${ }^{7}$ South Asian Clinical Toxicology of Research Collaboration, Peradeniya, Sri Lanka

Background: Snakebite envenoming is a neglected global public health issue with significant morbidity and mortality. The major life-threatening toxin-mediated clinical syndromes in Russell's viper envenoming are venom induced consumption coagulopathy (VICC), neurotoxicity and acute kidney injury. The current treatment with antivenoms has limited effectiveness as many envenoming syndromes are irreversible by the time antivenom is given. MicroRNAs are potential diagnostic biomarkers of several diseases. This study aimed to establish whether serum microRNAs can be used to identify Russell's viper envenoming.

Methods: This is a retrospective case study conducted in Sri Lanka. Early serum samples $(<2.5$ hours of post bite) from Russell's viper bites envenomed patients were used. We profiled circulating microRNAs from envenomed patients $(n=5)$ and healthy individuals $(n=4)$ through TaqMan discovery microRNAs OpenArray RT-qPCR platform (754 microRNAs). We selected 46 differentially expressed microRNAs and validated these in a larger cohort of patients $(n=40)$ and healthy controls ( $n=19)$ using a custom OpenArray panel.

Results: We identified early increases in many (61/176) microRNAs in snake bite patients in the discovery cohort. Thirty-one microRNAs (out of 46) were significantly upregulated in the validation cohort. Five microRNAs (miR-30c, miR-30b, miR-15b, miR-451and miR-181a) had greater diagnostic performance with receiver operator characteristics area-under-curve (ROC-AUC) $>0.85$. Pathway analysis of target mRNAs of these differentially expressed microRNAs is associated in coagulation and inflammation pathway in Russell's viper envenoming. 
Conclusion: Our preliminary data suggests envenoming increases miRNA expression in the circulation within less than 2.5 hours of post bite. We identified several microRNAs with good diagnostic performance in D. russellii envenoming. These urinary microRNAs have potential clinical application as early identification of D. russellii envenoming.

002. The Minor Cannabinoid Cannabigerol (CBG) is a Highly Specific Biomarker of Recent Cannabis Smoking

John M Rague ${ }^{1,2}$, George S Wang ${ }^{2}$, Ming $\mathrm{Ma}^{3}$, Gregory P Dooley ${ }^{4}$, Kyle Friedman $^{1}$, Ashley Brooks-Russell ${ }^{3}$, Michael J Kosnett ${ }^{2,3}$

${ }^{1}$ Rocky Mountain Poison and Drug Safety, Denver, Colorado, USA.

${ }^{2}$ University of Colorado School of Medicine, Aurora, Colorado, USA.

${ }^{3}$ Colorado School of Public Health, Aurora, Colorado, USA.

${ }^{4}$ Colorado State University, Fort Collins, Colorado, USA

Background: The determination of recent cannabis use is of forensic interest in the investigation of automotive crashes or workplace mishaps. Because THC may persist in blood after psychomotor effects of intoxication resolve, particularly in regular users, short-lived minor cannabinoids may offer advantages in assessment of recent use.

Research Question: How will blood concentration of cannabigerol (CBG), a natural constituent of cannabis, discriminate from recent smoking (within the past 30 minutes) in daily and occasional (use $1-2$ days/week over past 30 days) cannabis users?

Methods: As part of an observational cohort study, whole blood cannabinoids including CBG were measured by liquid chromatography with tandem mass spectrometry at baseline, and 30 minutes after initiation of a 15-minute supervised interval of ad-libitum smoking or vaping cannabis in occasional $(\mathrm{n}=24)$ and daily $(\mathrm{n}=32)$ adult cannabis users. Per protocol, subjects self-reported abstention from use of all cannabis for ${ }^{3} 8$ hours overnight before baseline measurement.

Results: Prior to ad-libitum smoking of cannabis, no occasional user ( $\mathrm{n}=$ 24) had a detectable concentration of CBG, while $25 \%$ had a detectable concentration of CBG after smoking cannabis. For daily cannabis users (n $=32$ ), $6.3 \%$ had a detectable CBG at baseline (LOD $0.2 \mathrm{ng} / \mathrm{ml}$ ), and $46.9 \%$ had detectable CBG after smoking cannabis. The odds ratio for recent cannabis smoking was 16.2 (95\% CI: 4.1, 64.4) for the finding of detectable CBG. In this mixed cohort of occasional and daily users, receiver operating characteristic curve analysis indicated that blood CBG concentration of $0.78 \mathrm{ng} / \mathrm{ml}$ had $96.4 \%$ specificity for identifying a $15-$ minute interval ad-libitum cannabis smoking initiated 30 minutes earlier. Conclusion: $\mathrm{CBG}$ has forensic utility as a specific, albeit insensitive biomarker of recent use. Study limitations include inability to supervise abstinence prior to baseline measurements, and the use of different selfsupplied cannabis strains by subjects.

003. Single Nucleotide Polymorphisms of Mu Opioid Receptor Gene OPRM1 in ED Patients with Acute Opioid Overdose

Alex F Manini, Lynne D Richardson, Carmen Vargas-Torres, Kavey Vidal, Yasmin L Hurd

Icahn School of Medicine at Mount Sinai, New York, NY, USA

Background: Genetic variations in the mu-opioid receptor (MOR) gene (OPRM1) mediate individual differences in opioid addiction. Several common MOR single nucleotide polymorphisms (SNP) have been previously associated with opioid addiction in humans.

Research Question: We studied whether three common MOR SNPs (rs3778150, rs1799971, rs2075572) were associated with overdose severity in patients presenting to the emergency department (ED) with acute opioid overdose.

Methods: This observational cohort at two urban teaching hospitals evaluated consecutive adult ED patients presenting with suspected acute opioid overdose over five years $(6 / 15-2 / 20)$. Specimens were linked with clinical variables then de-identified prior to genetic SNP analysis. The study outcome, overdose severity, was defined as the following composite outcome: in-hospital occurrence of either (A) respiratory arrest, or (B) cardiac arrest. Blinded Taqman genotyping of the SNPs were performed after standard DNA purification and whole genome amplification.

Results: We evaluated 331 patients (37.5\% female, mean age 45.7 (SD 16.6) of whom $64(19.3 \%)$ suffered severe outcomes. Urine toxicology was positive in $59.4 \%$, of which there were positives for 14 benzodiazepines, 15 cocaine, 22 opiates, 19 methadone, and 6 barbiturates. All genotypes examined conformed to Hardy-Weinberg equilibrium. When controlling for demographics, clustering by year, and number/type of drug exposures, the rs 2075572 variant allele was significantly associated with over 4-fold increased odds of overdose severity (aOR 4.49, CI 1.4913.54), while the rs 1799971 variant allele was associated with $43 \%$ decreased odds (aOR 0.57, CI 0.50-0.64). Finally, the rs3778150 ( $\mathrm{p}=0.96$ ) had no significant association with the study outcome.

Conclusion: These data demonstrate that in ED patients with acute opioid overdose, two OPRM1 SNPs were independent predictors of opioid overdose severity - the rs2075572 variant allele (higher severity) and the rs1799971 variant allele (lower severity). This confirms that OPRM1 SNP testing is a potential target for personalized medical prescribing practices with regard to overdose vulnerability.

\section{Prevalence Of Adulterants in Acute Opioid Toxic Patients}

Michael Levine ${ }^{1}$, Kim Aldy ${ }^{2,3}$, Alison Meyn ${ }^{4}$, Sharan Campleman ${ }^{2}$, Alex Krotulski ${ }^{5}$, Barry Logan ${ }^{5,6}$, Paul Wax ${ }^{2,3}$, Jeffrey Brent ${ }^{7}$, Alex Manini ${ }^{8}$, On Behalf of the Toxicology Investigators Consortium (ToxIC) Fentalog Study Group

${ }^{1}$ University of California, Los Angeles, Los Angeles, CA, USA. ${ }^{2}$ American College of Medical Toxicology, Phoenix, AZ, USA. ${ }^{3}$ University of Texas Southwestern Medical Center, Dallas, TX. ${ }^{4}$ American College of Medical Toxicology, Phoenix, AZ. ${ }^{5}$ Center for Forensic Science Research and Education at the Fredric Rieders Family Foundation, Willow Grove, PA. ${ }^{6}$ NMS Labs, Horsham, PA. ${ }^{7}$ University of Colorado School of Medicine, Aurora, CO. ${ }^{8}$ Icahn School of Medicine at Mount Sinai, New York, NY

Background: Adulterants may be contaminants from illicit manufacturing, added as bulking agents, and intentionally added for synergistic effects. We describe adulterants confirmed in a cohort of opioid overdose patients presenting to the emergency department (ED).

Hypothesis: This study sought to determine if the types of adulterants change over time or by region.

Methods: The Toxicology Investigators Consortium (ToxIC) Fentalog Study Group has an ongoing multi-center prospective case series of presumed opioid overdose patients presenting to nine US EDs. Discarded blood from adult patients with acute opioid overdose were collected and analyzed via liquid chromatography quadrupole time-of-flight mass spectrometry. Sites were located in three regions: West (California, Oregon); Central (Michigan, Missouri); East (Pennsylvania, New York, New Jersey). Cases were further analyzed via time; (period 1: September-December 2020; period two: January-April 2021; period 3: May-August 2021).

Results: A total of 378 subjects were identified; $68 \%$ male, median (IQR) age 40 (31-53). The following adulterants were found: Diphenhydramine $(15.6 \%)$, hydroxyzine (11.6\%), levamisole (23.0\%), local anesthetics (23.6\%), phenacetin $(18.5 \%)$, and quinine $(26.4 \%)$. Regional variation occured for both levamisole and quinine. Levamisole was found in one (2.7\%) Western sample vs. 32 (29.6\%) Central US samples and 54 (23.2\%) Eastern samples ( $\mathrm{p}=0.004 ; 95 \%$ CI 8.3-26.7\%). Quinine was more commonly encountered in the Central US; 1 (2.7\%) Western samples vs. 54 (50\%) Central US samples and $45(19.3 \%)$ Eastern samples ( $\mathrm{p}=0.013$; $95 \%$ CI $4.5-22.2 \%)$. Diphenhydramine use increased with time; 8 (9.4\%) samples from period one vs. 23 (20\%) samples from period three $(\mathrm{p}=0.04 ; 95 \%$ CI $0.36-20.0)$. Quinine also increased over time: $12(14.1 \%)$ in period one vs. $43(37.3 \%)$ in period three. Phenacetin decreased over time; $21(24.7 \%)$ in period one vs. $14(12.2 \%)$ in period three $(\mathrm{p}=0.0021 ; 95 \%$ CI 1.76-23.7). 
Conclusion: Adulterants are commonly encountered in opioid overdose and change over time and location.

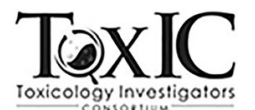
This research was performed in collaboration with the ACMT Toxicology
Investigators Consortium.

005. Urinary Concentrations of Endocrine-Disrupting Metals and Their Association With Breast Cancer in US Women

Belinda Bell ${ }^{1}$, Jianmin $\mathrm{Zhu}^{2}$, Yudan Wei ${ }^{1}$

${ }^{1}$ Mercer University School of Medicine, Macon, GA, USA. ${ }^{2}$ Fort Valley State University, Fort Valley, GA, USA

Background: Breast cancer is a heterogenous cancer with an increased incidence in industrialized regions and urban areas. There has been an increasing concern about the role of environmental metal exposure in breast cancer development.

Hypothesis: Is there an association between urinary concentrations of cadmium, lead, and mercury and prevalent breast cancer among US women?

Methods: This is a cross-sectional study that analyzed female participants, aged 20 years or older, obtained from the 2007 to 2016 National Health and Nutrition Examination Survey (NHANES). In the NHANES, spot urine samples were collected, and metal concentrations were measured using inductively coupled plasma mass spectrometry. To control for urine dilutions in spot urine samples, urine creatinine-corrected concentrations of metals were determined in the study. Self-reported cancer diagnosis was obtained through a medical conditions' questionnaire. Multivariate logistic regression models were constructed to examine the association between urinary concentrations of metals and prevalent breast cancer, adjusting for potential confounders including demographic, behavioral, and reproductive health variables.

Results: Among the 3,352 study participants, 106 reported having breast cancer (weighted prevalence, $3.13 \%$ ). Significantly increased urinary concentrations of lead and cadmium were observed in women with breast cancer ( $p$ $<0.0001$ ), compared to those without breast cancer. After adjusting for all the covariates included in the study, only urinary lead was shown to be significantly associated with breast cancer. The highest quartile of urinary lead $(\geq$ $0.71 \mu \mathrm{g} / \mathrm{g}$ creatinine) was associated with a 2.95 -fold $(95 \%$ CI: $1.13,7.70)$ increased odds of female breast cancer. There were no significant associations seen between urine cadmium or mercury levels and breast cancer.

Conclusion: This study demonstrated a potential relationship between lead exposure, measured as urinary lead concentrations, and prevalent breast cancer among US women. Prospective and mechanistic studies are warranted to further investigate this relationship and explore the role of lead in breast carcinogenesis.

006. Evaluating Public Perceptions of Expanded Access to Naloxone William Eggleston

Binghamton University School of Pharmacy \& Pharmaceutical Sciences, Binghamton, NY, USA

Background: Increased access to naloxone is a priority intervention for reducing opioid overdose deaths. Little is known about public perceptions of novel naloxone distribution strategies.

Research Question: What are community perceptions of novel naloxone distribution models?

Methods: This is a prospective survey analysis of community member perceptions of novel naloxone distribution models. The study was deemed exempt by the Binghamton University IRB. We developed a survey, in conjunction with regional harm reduction groups, to gauge community member perceptions of novel naloxone distribution models being used in our own community, in other US communities, or that had been proposed by harm reduction experts. Distribution models included online, pharmacy over-thecounter, grocery store, prescription only, vending machine, and grocery stores. Participants were recruited from public spaces (shopping malls, community events, etc.) and asked to complete the survey after providing consent.
Participants reported basic demographic information, opioid use and overdose history, naloxone use history, perceptions about naloxone, and perceptions about novel distribution models. Descriptive statistics were used to evaluate demographics data and unpaired t-tests were used for comparisons.

Results: Eighty-six participants were enrolled in the study. The population was $66.3 \%$ female, $75.6 \%$ White, $17.4 \%$ rural, had a median age of 41.5 years, and $47.7 \%$ had previously been prescribed an opioid medication. A minority of participants agreed that naloxone should be available online $(13.6 \%)$, over-thecounter $(36.4 \%)$, in grocery stores $(14.2 \%)$, from vending machines $(6.8 \%)$, or in gas stations $(9.9 \%)$. Gender, race, and previously witnessing an overdose were not associated with a difference in over-the-counter, online, or other novel distribution models. A previous opioid prescription was associated with increased support for over-the-counter naloxone $(\mathrm{p}=0.0163)$.

Conclusion: Although increased access to naloxone reduces overdose deaths, participants were largely unsupportive of novel distribution models. More research is needed to increase community acceptance of novel naloxone distribution mechanisms.

007. A Decade in Review: Trends in Female Authorship in Two PeerReviewed Toxicology Journals

Jennifer S Love ${ }^{1}$, Lauren Murphy ${ }^{2}$, Courtney Temple ${ }^{3,4}$, Meghan B Spyres $^{5,6}$, Ayrn D O'Connor ${ }^{5,6}$

${ }^{I}$ Icahn School of Medicine at Mount Sinai, New York, NY, USA. ${ }^{2}$ Temple University, Philadelphia, Pennsylvania, USA. ${ }^{3}$ Oregon Poison Center, Portland, OR, USA. ${ }^{4}$ Oregon Health \& Science University, Portland, OR, USA. ${ }^{5}$ Banner University Medical Center Phoenix, Department of Medical Toxicology, Phoenix, AZ, USA. ${ }^{6}$ University of Arizona College of Medicine Phoenix, Department of Emergency Medicine and Internal Medicine, Phoenix, AZ, USA

Background: The gender gap in academic medical literature is well established. As of 2019, $42 \%$ of US board-certified toxicologists are women, but no studies have evaluated gender distribution of authorship in peer-reviewed toxicology journals. This study sought to characterize gender authorship and analyze trends over a decade in two toxicology journals.

Hypothesis: Female first and senior authorship is proportionally lower than male authorship although it will increase over the study period.

Methods: All non-abstract publications in the Journal of Medical Toxicology and Clinical Toxicology from 2011-2020 were retrospectively reviewed using Gender-API, a gender identification tool, to determine author male/female gender. First and senior (last) author names, article type, and publication year were recorded. Frequency distributions, chisquare and odds ratios analyses were performed using SAS ®.

Results: Two thousand one hundred seventy three publications were reviewed with 1994 included in final analysis. Six hundred twenty-six (31.4\%) publications with female first authors and 342 (19.3\%) with female senior authors were identified. There were twice as many male-identified first authors $(68.6 \%)$ compared to female-identified first authors and more than four times as many male-identified senior authors $(80.8 \%)$. The proportion of first and senior authors identified as 'female' increased over time ( $23.3 \%$ and $16.9 \%$ in 2011 vs. $31.4 \%$ and $22.2 \%$ in 2020 , respectively) The proportion of male-male author dyads varied; female-male author dyads increased between $2011(17.6 \%)$ and 2017 (32.7\%). The percentage of female-female dyads remained low ( $\max 10.3 \%$ in 2018$)$. While $42.0 \%$ of research studies and $39.6 \%$ of case reports had female first authors, only $16.7 \%$ of research studies and $19.3 \%$ of case reports had female senior authors.

Conclusion: The proportion of female first and senior authors increased over time but was significantly lower than male authors in similar author positions. Awareness of authorship trends encourages gender equity in toxicology and promotes dedicated mentorship of women in research and publication.

008. Establishment of a Novel Electronic Consult Medical Toxicology Service

Avery Michienzi, Samuel Collins, Kimberly Dowdell, Christopher Holstege University of Virginia, Charlottesville, VA, USA 
Background: Electronic consults (eConsults) are a billable service meant as a platform for primary care providers to ask focused questions to specialists. The goal of eConsults is to increase timely access to specialists, improve provider education, and avoid unnecessary referrals to specialty clinics. This is the first known description of a clinical toxicology eConsult service.

Research question: What are the most asked questions from outpatient providers to toxicologists?

Methods: This is a descriptive cross-sectional review of the eConsult Medical Toxicology service over three years. The Medical Toxicology eConsult service was developed by a public university's Medical Toxicology Division in collaboration with primary care and telehealth services. The team created templates to facilitate outpatient eConsults to ensure the service was used for non-emergent care.

Results: From 2018 to 2021, there were 53 Medical Toxicology eConsults. Consult topics included: UDS interpretation (33); heavy metal lab interpretation (9); follow-up snake bites care (2); herbal supplements (2); benzene (1); epoxy (1); pesticide (1); nitrous oxide (1); baclofen taper (1); nonspecific occupational exposure (1); concern for intentional poisoning (1). Of these consults, 43 were completed with electronic eConsult notes, six were determined to be too complex and referred to the Medical Toxicology clinic, one was referred to dermatology clinic, one was referred to pain management, one was declined due to acuity, and one was declined due to lack of appropriate information.

Conclusion: This is the first known eConsult service established for the specialty of Medical Toxicology. The most frequently asked questions involved interpretation of urine drug screens and heavy metal testing. Misinterpretation of these tests can lead to inappropriate management by primary care and further unnecessary testing. Access to a Medical Toxicologist's expertise rapidly via brief eConsults can significantly impact patient care and provide formal documentation directly into the electronic medical record.

\section{Lightning Orals, Abstracts 009-015 \\ 009. Abstract Withdrawn}

010. Universal Liquid Chromatography Tandem Mass Spectrometry Urine Drug Screening in Routine Prenatal Visits for Pregnant Patients

David B Liss, Bridget M Galati, Stephen M Roper, Rachel M Ancona, Jeannie C Kelly, Grace Godsy, Cynthia E Rogers, Evan S Schwarz Washington University, Saint Louis, MO, USA

Background: Drug use in pregnancy is a persistent concern. Universal screening by validated questionnaire is recommended, however universal urine drug screening is not.

Research Question: What is the prevalence of positive results on a liquid chromatography tandem mass spectrometry (LC-MS/MS) drug screen in a sample of pregnant patients not already enrolled in a substance use treatment program?

Methods: This is a cross-sectional study of pregnant patients presenting at an urban, academic medical center during August 2020-March 2021. We included patients of obstetrics clinics and utilized surplus urine leftover from routine care. Patients who were not pregnant or enrolled in treatment for a substance use disorder (SUD) were excluded and duplicate samples discarded. The primary outcome is a positive result from LC-MS/MS analysis of urine for one of 48 substances including drugs of abuse, prescription medications, and drug metabolites. The analysis is descriptive.

Results: We obtained 1771 urine samples, 970 were excluded (47, 4.8\% for enrollment in SUD treatment), leaving 801 unique patients included in the study. There were 267 (33.3\%, 95\% CI: $30.1-36.7, \mathrm{p}<0.001)$ patients with a positive result. Drugs of abuse potential detected included the following: oxycodone $2(0.3 \%)$, morphine $0(0.0 \%)$, 6-acetylmorphine $0(0.0 \%)$, fentanyl $3(0.4 \%)$, hydrocodone $2(0.3 \%)$, hydromorphone $1(0.1 \%)$, methadone 1 $(0.1 \%)$, benzoylecgonine $11(1.4 \%)$, methamphetamine $10(1.3 \%)$, 9$\mathrm{COOH}$-tetrahydrocannabinol-glucuronide 244 (30.5\%).
Conclusion: The point prevalence of positive urine tests for drugs of abuse potential among pregnant patients in this sample was low except for very high rates of cannabis detected. It is possible that pregnant patients using fentanyl, cocaine, or methamphetamine either do not attend our obstetric clinics or that these patients were already referred to our internal SUD clinic for pregnant patients. The findings of this singlecenter pilot study may not be generalizable to other obstetric clinic populations.

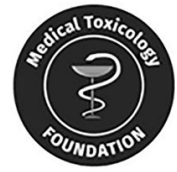

This research was supported by a 2020 Medical Toxicology Foundation Innovative Research and Teaching Award

011. Mitigating Dietary Supplement Risk through Community Targeted Education and Product Testing

Melissa Givens ${ }^{1}$, Abraham R Walter ${ }^{2,1}$, Cindy Crawford ${ }^{2,1}$, Bharathi Avula $^{3}$, Andrea Linsey ${ }^{2,1}$, Aimee Hunter ${ }^{4}$, Ikhlas A Khan ${ }^{3}$, Patricia A Deuster $^{1}$

${ }^{1}$ Consortium for Health and Military Performance, Department of Military and Emergency Medicine, F. Edward Hébert School of Medicine, Uniformed Services University, Bethesda, MD, USA. ${ }^{2}$ Henry $M$ Jackson Foundation for the Advancement of Military Medicine, Bethesda, MD, USA. ${ }^{3}$ National Center for Natural Products Research, School of Pharmacy, University of Mississippi, University, MS. ${ }^{4}$ Department of Laboratory Animal Resources, Uniformed Services University, Bethesda, MD, USA

Background: Dietary supplement use is ubiquitous with a higher prevalence of use reported in first responder and military communities. Some dietary supplements may be adulterated or misbranded, which poses a risk to community members. Operation Supplement Safety is a Department of Defense program that provides evidence-based information about dietary supplements to Service Members, their families, healthcare providers, and leaders.

Hypothesis: Targeted education coupled with a community focused inquiry provide a means to conduct content analysis on dietary supplements of interest to the community and inform relative risk.

Methods: Dietary supplement products frequently asked about through the Operation Supplement Safety (@OPSS.org) Ask-the-Expert portal were selected. Product analyses were performed to verify whether selected products were accurately labeled according to the Supplement Facts listed ingredients and identify any risky ingredients contained in the products.

Results: Forty-one unique dietary supplement products were selected for analysis. Twelve (29.3\%) were marketed as pre-workout products for increased energy and focus; 14 (34.1\%) for weight loss; four $(9.8 \%)$ for male enhancement/testosterone boosters; and $11(26.8 \%)$ as body building supplements. Only $11(26.8 \%)$ dietary supplement products had accurate labels. Twenty-six (63.4\%) products were misbranded, $10(24.4 \%)$ products were adulterated, and six products were both misbranded and adulterated. Risky ingredients appeared on $23(56 \%)$ product labels and analyses revealed eight products contained additional risky ingredients (to include Scheduled III drugs) not listed on the labels.

Conclusion: Operation Supplement Safety's resources provide a responsive platform that facilitates community-based identification of dietary supplement products of interest and enables focused educational efforts. Targeting supplements of community interest informs risk mitigation through content analysis that would otherwise be untenable in a market with countless products. Operation Supplement Safety or similar platforms should be considered for other communities with a high prevalence of supplement use, such as police and firefighters. 
012. Increased Use of Telemedicine and Other Changes in Toxicology Billing and Coding During the Mid Covid-19 Pandemic Peter W Crane, MD, MBA, RN, FACEP, Timothy J Wiegand, MD, FACMT, FAACT, DFASM

University of Rochester Medical Center, Rochester, NY, USA

Background: The success of any medical service line correlates with the ability to demonstrate value including revenue generation. We previously reported our Current Procedural Terminology (CPT) patterns for toxicology billing pre Covid-19; which consisted of almost 40 different CPT codes. The COVID-19 pandemic led to changes in practice and billing that we suspect are widespread throughout the country. We describe our billing experience including changing CPT utilization during the midpandemic time frame.

Hypothesis: COVID-19 pandemic impacted toxicology billing practices with increased use of telemedicine encounters and CPT coding.

Methods: This was a convenience sample of all CPT codes billed for toxicology services during October/November 2020. Data was derived from QA processes and standard billing databases for a busy urban academic institution. No identifiable patient information was collected.

Results: Of 1853 toxicology encounters during this time 12 CPT codes (99291, 99292, 99285, 99223, 99451, 99214, 99214, 99215, 99356, 99233, 99406, 99213 and 992220 accounted for $89.65 \%$ of total billing codes with 27 overall total codes utilized. Telehealth specific CPT codes (G0426, 9947, 9948, 99451, and 99452) accounted for 5.1\% of toxicology encounters; however, all office-based encounters were conducted as telehealth during this time due to the pandemic $(99213,99214,99215,99204$, 99205) and translated to the appropriate telehealth billing codes. After correction, total telehealth visits accounted for $19.81 \%$ of all encounters. Conclusion: The pandemic resulted in a notable shift in our billing practices. While we utilized a reduced total coding spectrum of $27 \mathrm{CPT}$ codes, 12 individual codes covered the majority of encounters. Telehealth encounters increased to nearly $20 \%$ of all CPT codes, primarily to conduct office visits, rather than in person. While more research needs to be done, prior research illustrated that telehealth can be a viable method of revenue generation for the toxicology service line.

013. Ivermectin Associated Adverse Events in the Treatment and Prophylaxis of COVID-19 Reported to the FDA ACMT COVID-19 ToxIC (FACT) Pharmacovigilance

Rita Farah $^{1,2}$, Ziad Kazzi $^{1,2}$, Jeffrey Brent ${ }^{3}$, Keith Burkhart ${ }^{4}$, Paul Wax ${ }^{5,6}$, Kim Aldy ${ }^{5,6}$, On Behalf of the Toxicology Investigators Consortium (ToxIC) FACT Study Group

${ }^{1}$ Department of Emergency Medicine, Emory University, Atlanta, GA, USA. ${ }^{2}$ Georgia Poison Center, Atlanta, GA, USA. ${ }^{3}$ University of Colorado, School of Medicine, Aurora, CO, USA. ${ }^{4}$ Center for Drug Evaluation and Research, United States Food and Drug Administration, Silver Spring, MD, USA. ${ }^{5}$ American College of Medical Toxicology, Phoenix, AZ, USA. ${ }^{6}$ University of Texas Southwestern Medical Center, Dallas, TX, USA

Background: Since the beginning of the COVID-19 pandemic, there has been an interest in repurposing currently available pharmaceuticals, such as ivermectin.

Methods: This is a prospective case series of adverse events (AEs) submitted to the FDA ACMT COVID-19 ToxIC (FACT) Pharmacovigilance Project between $11 / 23 / 20$ and $8 / 30 / 21$. FACT is an active surveillance project with 15 participating medical centers focusing on identifying possible AEs related to any medication or substance administered with intent to treat or prevent COVID-19 infection. Cases were included if the implicated substance of the AE was identified as ivermectin.

Results: A total of 42 cases of self-medication with ivermectin were reported to the FACT Pharmacovigilance Project during the study period. The median age was 53 years [range $39-65$ ]; $48 \%$ were female. Selfmedication with veterinary paste and injectable formulations were the most cited sources of ivermectin. Doses ranged from $12 \mathrm{mg}$ to 1360 mg. Patients reported using ivermectin for prophylaxis $(60 \%)$, for treatment of documented COVID-19 (21\%), and for treatment of symptoms of undocumented COVID-19 (14\%). Thirty patients (71\%) presented to emergency departments and ten patients $(24 \%)$ were admitted to the hospital. Neurological toxicity was the most frequent finding, ranging from headache $(10 \%)$, numbness $(5 \%)$, weakness $(5 \%)$, tremors $(7 \%)$, dizziness $(21 \%)$, to hallucinations $(5 \%)$, altered mental status $(29 \%)$, confusion $(21 \%)$, visual disturbances and visual hallucinations $(12 \%)$ and seizures (7\%). Gastrointestinal symptoms (24\%) were also reported. Three patients who took a veterinary parenteral preparation containing propylene glycol (50-60\%) orally developed lactic acidosis, hypotension and tachycardia.

Conclusion: Ivermectin use for the attempted treatment and prophylaxis of COVID-19, despite its lack of documented efficacy, has the potential for serious adverse health effects. This is especially true when patients are self-treating with this medication and when they are using formulations with large doses for multiple days intended for use in large animals.

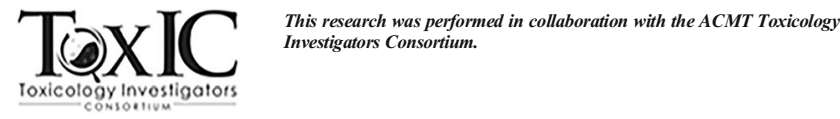

014. Increased Risk of COVID-19 Mortality in Patients With Opioid Use Disorder in an Urban Hospital System Jonathan Schimmel ${ }^{1}$, George Loo ${ }^{1}$, Ka Ming Ngai ${ }^{1}$, Alex F Manini ${ }^{1,2}$ ${ }^{I}$ Department of Emergency Medicine, Icahn School of Medicine at Mount Sinai, New York, NY, USA. ${ }^{2}$ Division of Medical Toxicology, Icahn School of Medicine at Mount Sinai, Elmhurst Hospital Center, New York, NY, USA

Background: There has been concern that the 2019 novel coronavirus (COVID-19) may disproportionately impact populations with substance use disorders. Worsening COVID-19 severity in patients with opioid use disorder (OUD) is plausible based on biological mechanisms (respiratory effects, immune mechanisms, drug-drug interactions) and social/ environmental factors. This study examined COVID-19 outcomes in patients with OUD. The hypothesis was that among Emergency Department (ED) and hospitalized patients with COVID-19, OUD is associated with a higher incidence of COVID-19 mortality.

Methods: This was a retrospective cohort study at an urban academic health system (five hospitals) and used automated chart abstraction for a 17 -month period (3/2/2020 - 8/4/2021). Adult ED or hospitalized patients ( $\geq 18$ years) with positive SARS-CoV-2 PCR tests were included. The primary outcome was COVID-19 mortality, defined as in-hospital allcause mortality. OUD was defined by ICD-10 diagnostic codes (F11) or Medications for OUD in the medical record (prescribed in-hospital or outpatient). Opioid overdose was defined as naloxone administration within 24 hours of hospitalization or ED stay. Post-hoc power analysis demonstrated $84.9 \%$ power to demonstrate a $5 \%$ increase in mortality risk, based on a sample size of 19,769, assuming $1.9 \%$ had OUD, and a $10.6 \%$ baseline outcome rate.

Results: Of 19,769 patients (median age 59 years, $48.4 \%$ female, $24.5 \%$ Black, 26.7\% Hispanic), 374 (1.9\%) had OUD, and 21 (0.1\%) had opioid overdose. There were $2,103(10.6 \%)$ in-hospital deaths. Bivariate analysis found both OUD (OR 2.2, p < 0.001) and opioid overdose (OR 1.27, p = 0.012 ) were associated with mortality. Multivariable logistic regression controlled for demographics (age, sex, race/ethnicity), smoking, and obesity, and found OUD was an independent predictor of COVID-19 mortality (aOR 3.5 at the mean age, 95\% CI 2.6 - 4.7).

Conclusion: In this cohort, OUD was an independent predictor of COVID-19 mortality. Opioid overdose was associated with COVID-19 mortality, but its rarity prevented robust modeling. 
015. Impact of the COVID-19 Pandemic on Emergency Department Alcohol Intoxication Presentations and Positive Screens for Problem Drinking

Maryann Mazer-Amirshahi ${ }^{1,2}$, Ronak Sutariya ${ }^{2}$, Sameer Desale ${ }^{1}$, Mihriye Mete ${ }^{1}$

${ }^{1}$ MedStar Health, Washington, DC, USA. ${ }^{2}$ Georgetown University, Washington, DC, USA

Background: The effects of the COVID-19 pandemic can exacerbate alcohol use disorder and increase problem drinking; yet access to psychosocial support and medical care may be limited.

Hypothesis: There would be an increase in the proportion of patients presenting with acute alcohol intoxication and positive screens for problem drinking during the COVID-19 pandemic compared to before the pandemic. Methods: Retrospective chart review of emergency department (ED) visits from nine hospitals in the DC/Baltimore area from January 2019 through June 2021. The health system utilizes a validated system for Screening, Brief Intervention, and Referral to Treatment (SBIRT) for ED patients that are clinically stable and willing to complete screening. We evaluated trends in patients that had a positive SBIRT screen for problem drinking (AUDIT-C score $\geq$ three in women, four in men) and those presenting with acute alcohol intoxication before March 2020 and during the COVID pandemic (after March 1, 2020).

Results: There were 543,223 visits with screens for patients aged 12 to 95 years during the study period (230,559 pre-COVID, 312,664 during). The population was primarily African American (61\%) and 56\% were female. Visits for intoxication or a positive screen for problem drinking increased from $8.8 \%$ pre-COVID to $9.7 \%$ during COVID ( $\mathrm{p}=<0.001$ ). Acute alcohol intoxication presentations increased as a proportion of all visits from $1.5 \%$ to $1.8 \%(\mathrm{p}<0.001)$. Presentations for acute alcohol intoxication increased as a percentage of all alcohol-related positive screens during the COVID pandemic (17.1 vs. $18.8 \%, \mathrm{p}<0.001)$. A higher percentage of patients had an AUDIT-C score consistent with problem drinking during the COVID-19 pandemic $(7.4 \%$ vs. $8.0 \%$, p < 0.001$)$.

Conclusion: There was an increase in positive SBIRT screens as well as the proportion of visits for acute alcohol intoxication during the COVID19 pandemic. Additional research should focus on mitigation strategies to address alcohol use during this vulnerable time.

\section{Moderated Posters, Abstracts 016-040}

016. Identifying Missed Cases of Serotonin Syndrome: A Novel Electronic Health Record Search Method

Jacob T Howshar ${ }^{1}$, C William Heise ${ }^{1,2}$, Pooja Rangan ${ }^{1,2}$

${ }^{1}$ University of Arizona College of Medicine Phoenix, Phoenix, AZ, USA.

${ }^{2}$ Banner University Medical Center, Phoenix, AZ, USA

Background: Serotonin syndrome (SS) is a rare but life-threatening adverse drug reaction characterized by a diagnostic triad of neuromuscular agitation, autonomic hyperactivity, and altered mental status. SS is notoriously difficult to diagnose because of its variation in symptoms and severity.

Hypothesis: A novel electronic health record (EHR) query can be designed to identify undiagnosed cases of SS based on ICD-10 coding of symptom combinations.

Methods: This study is a retrospective chart review of adult patients hospitalized in Banner Health facilities between 2017-2021. An EHR query was designed that utilized diagnostic coding to find encounters suggestive of SS but lacking the diagnosis. Patients prescribed proserotonergic medications and who were diagnosed with at least one symptom from each category in the characteristic SS triad during a single inpatient encounter were identified. A representative sample was randomly selected for detailed chart review and confirmed by expert evaluation as a truly missed case of SS or a false positive.

Results: A total of 211 encounters were identified and 101 were reviewed. Of those, 60 were true missed cases of SS and 41 were false positives. Chi- square analysis showed that gender, age, mood disorders, and substance abuse disorders were not significant predictors of true missed cases or false positives, but logistic regression indicated that our tool was more predictive in younger individuals.

Conclusion: Our study shows that serotonin syndrome is frequently missed by physicians. From 2017-2021, there were only 37 diagnosed cases of SS, yet our scoring tool identified approximately 120 additional cases. Although our search method lacked specificity, it provided a dramatically enriched sample from which undiagnosed cases could be found. Our novel strategy of hunting for missed cases using diagnostic coding can be invaluable for further investigations on serotonin syndrome and can be readily extrapolated to other rare conditions.

017. Oral Potassium Exposure: A Retrospective Review of The National Poison Data System 2010-2021

Rita Farah $^{1,2}$, Brent W Morgan ${ }^{1,2}$, Robert J Geller ${ }^{2,3}$, Stephanie Hon ${ }^{2}$, Joe E Carpenter ${ }^{1,2}$

${ }^{1}$ Department of Emergency Medicine, Emory University, Atlanta, GA, USA. ${ }^{2}$ Georgia Poison Center, Atlanta, GA, USA. ${ }^{3}$ Department of Pediatrics, Emory University School of Medicine, Atlanta, GA, USA

Background: Literature on oral potassium overdose is limited to case reports or case series of up to 13 cases. We aim to describe oral potassium exposures by demographics, clinical features and management.

Methods: This was a retrospective review of the National Poison Data System (NPDS) from January 2010 to June 2021. Cases involving singleagent oral potassium salts were included. Cases with unknown outcome were excluded from the analysis.

Results: During the study period, 1741 cases involving single-agent potassium ingestion were reported to NPDS. These incidents occurred almost equally among the adult (51.8\%) and the pediatric populations (48.2\%). Among the adult population, the median number of tablets was two (IQR: 1-8) with a maximum of 500 tablets; the median age was 55 years (IQR: 38-69). More than half of the cases (58\%) were deemed non-toxic or resulted in no effect, while $21 \%$ resulted in serious outcomes (moderate effects, major effects, or death). Hemodialysis was performed in twenty-four cases $(2.7 \%)$ and was significantly associated with the ingestion of a higher number of tablets $(5$ IQR: $2-16$ vs. 30 IQR:20-100; $p<0.001$ ) and with intentional use ( $p<0.001)$. Whole bowel irrigation and treatment with insulin and calcium were significantly associated with a more severe outcome.

Among children aged less than seven years, all reported exposures were unintentional, whereas, among those aged seven to 12 years and 13 to 17 years, suicidal exposures were reported in $15 \%$ and $77 \%$ of the cases, respectively. Ninety-three percent of pediatric exposures resulted in no effect and no major outcome occurred in this group. Surprisingly, $90 \%$ of suspected suicide occurred among females aged between 12 and 17 years. Conclusion: Preliminary analysis showed that whole bowel irrigation, hemodialysis, and treatment with insulin and calcium were significantly associated with more severe outcomes among adults. We found an alarming preponderance of suspected suicide among female teenagers.

018. Fear the Reaper: Symptomatic "One-Chip Challenge" Cases Reported to Philadelphia and Florida Poison Centers, Fall 2021

Wendy Stephan ${ }^{1}$, Lauren Longo ${ }^{2}$, Chiemela B Ubani ${ }^{3}$, Maurgerite Pacholski ${ }^{2}$, Richard Weisman ${ }^{1}$, Kevin C Osterhoudt ${ }^{2,4}$

${ }^{1}$ Florida Poison Information Center, Miami, FL, USA. ${ }^{2}$ The Poison Control Center at Children's Hospital of Philadelphia, Philadelphia, PA, USA. ${ }^{3}$ Florida/USVI Poison Information Center, Jacksonville, FL, USA. ${ }^{4}$ CHOP Center for Injury Research and Prevention, Philadelphia, $P A, U S A$

Background: Poison control centers receive calls about potentially toxic social media "challenges." In pre-Halloween marketing, one such challenge involved eating a single tortilla chip spiced with extremely hot Carolina Reaper and Scorpion Chile peppers; its packaging warns to "seek medical assistance should you experience difficulty breathing, fainting or extended nausea. 
Research Question: Did the hot chip challenge lead to use of poison control services?

Methods: Cases of capsaicin injury due to a commercial "One Chip Challenge", as reported to The Poison Control Center at Children's Hospital of Philadelphia, and to the Florida Poison Control Center Network, were reviewed from September 1 to October 31, 2021. Patient demographics, call site, and clinical effects were descriptively analyzed. Blinded, aggregation of pre-existing data was deemed exempt from human protections committee oversight.

Results: 30 symptomatic cases were reported over two months; 16 in eastern Pennsylvania and 14 in Florida. Age range was nine to 41 years (mean 14 years; $28 / 30$ were $<16$ years). Twenty-eight out of 30 were male. $80 \%$ of calls originated from schools and described students experiencing pain and/or vomiting. Overall symptoms recorded included oral burning (46\%), abdominal pain (43\%), vomiting (23\%), and eye pain (10\%). One case was referred to a hospital for concern of asthma exacerbation.

Conclusion: The commercially driven "One Chip Challenge" led to calls for help for poisoning injury, burdened school nurses, utilized poison control services, and resulted in ED evaluation. As a "performative" challenge, injured individuals tended to be early adolescent and male, and the act tended to occur in group settings. This study involved a small subset of U.S. poison centers; evidence of additional injuries can be viewed on selfposted internet videos. Policy should be considered related to the enticement of young people with challenges that include expectations that some participants are likely to warrant seeking medical assistance.

\section{Characterization of Tranexamic Acid Exposures Reported to the California Poison Control System}

Stacy Marshall ${ }^{1}$, Justin Lewis ${ }^{2}$, Timothy Albertson ${ }^{2}$, James Chenoweth ${ }^{1}$

${ }^{1}$ University of California Davis Medical Center, Sacramento, CA, USA.

${ }^{2}$ California Poison Control System, Sacramento, CA, USA

Background: Tranexamic acid (TXA) is a commonly used medication that acts by decreasing fibrinolysis. It is available as an oral tablet, topical preparation, and intravenous solution. Very limited data exists on TXA exposure and overdose.

Methods: This is a retrospective observation study of TXA exposures reported to the California Poison Control System (CPCS) between 1997 and 2021. Cases were excluded if TXA was used appropriately with no adverse event. Descriptive statistics were carried out to characterize the exposures.

Results: A total of 48 cases met inclusion criteria. Most $(41,85.4 \%)$ were oral exposures. There were also two subcutaneous exposures where TXA was given inadvertently, two intravenous exposures, two intrathecal exposures where TXA was given inadvertently, and one ocular exposure. Two patients with oral exposures required hospitalization, however both involved co-ingestions. Of the remaining oral exposure cases, $28(68 \%)$ were treated at home, six (14\%) were treated in the emergency room (ER) and released, and four $(10 \%)$ were referred to the ER. Two patients were receiving spinal blocks for joint arthroplasty when TXA was inadvertently administered intrathecally. One patient developed status epilepticus requiring pentobarbital infusion. A second patient with intrathecal TXA exposure required intubation, aggressive seizure management, and cerebrospinal fluid (CSF) exchange.

Conclusion: TXA exposures rarely result in serious adverse events via the oral route but can cause serious adverse events when mistakenly given intrathecally. Case reports have suggested that erroneous intrathecal administration of TXA is due to the similarity in appearance between TXA and local anesthetic ampules. Management of oral administration is supportive while intrathecal administration should focus on early identification of seizures, aggressive seizure control, and consideration of CSF exchange.

\section{Relative Lethality of Hypertension Drugs}

Dan Giurca ${ }^{1}$, Michael Hodgman ${ }^{2}$

${ }^{1}$ New York State Office of Mental Health, Albany, NY, USA. ${ }^{2}$ Upstate

New York Poison Center, Syracuse, NY, USA
Background: Suicidality and hypertension are prevalent problems. Accidental and intentional overdose with anti-hypertensives is common. Identification of anti-hypertensives with a safer risk profile following overdose may significantly decrease serious outcomes globally.

Research Question: Can the relative lethality (RL) of anti-hypertensives predict overdose relative morbidity (RM)?

Methods: The National Poison Data System was queried for single drug exposures to anti-hypertensives for the years 2006-2018. Specific generic codes for ACEI, ARB, BB, CCB and miscellaneous diuretics were searched. Demographics, specific product code, reason for exposure and outcomes were extracted. The RM of each anti-hypertensive was calculated as serious outcomes $(\mathrm{SO}=$ moderate + major + death $)$ divided by total exposures. The RL equation $(\mathrm{RL}=310 \times$ dose $/ \mathrm{LD} 50)$ represents the ratio of a 30-day supply of medication, to the human equivalent LD50 (derived from rat oral LD50 with FDA correction factor 6.2). Linear regression models were used to determine correlation between $\mathrm{RL}$ and RM.

Results: The most dangerous anti-hypertensive is verapamil $(\mathrm{RM}=28.50 \%)$. RM ranges are CCB $(11-28 \%)$, BB (5-22\%), ARB (5$13 \%)$, and ACEI (1-7\%). The CCB regression model shows strong correlation and implied causality between $R L$ and $R M\left(R^{2}=0.652, p=\right.$ 0.015). Overall, drugs with a lower RL had a lower RM.

Limitations: The average prescribed dose for each drug is unknown so the RL was calculated based on the maximum daily recommended dose. Age, sex, weight, co-morbidities, and average ingested dose determine $\mathrm{RM}$. The use of rat LD50 may not accurately reflect human toxicity.

Conclusion: As a class $\mathrm{CCB}$ and $\mathrm{BB}$ have the largest RM. RL can predict RM. This may be a useful tool for assessing overdose danger of newly introduced anti-hypertensives. The use of medications with a lower RL may reduce serious outcomes globally.

021. Drug And Toxicant-associated Rhabdomyolysis: Clinical and Epidemiologic Trends

Samy Chettat, Jennifer Stephani, Robert G Hendrickson, , On Behalf of the Toxicology Investigators Consortium (ToxIC)

Oregon Health and Sciences University, Portland, OR, USA

Background: Rhabdomyolysis is the breakdown of muscle tissue that may develop from many underlying causes (e.g. Toxicants, environmental, traumatic, exertional). Toxicant-induced rhabdomyolysis may occur from sedation with compressive ischemia of muscles or from psychomotor agitation with muscular flexion. Little research exists to describe the clinical aspects of toxicant-associated rhabdomyolysis and differences between rhabdomyolysis associated with sedation or agitation.

Research Question: What are the epidemiologic and clinical features of toxicant induced rhabdomyolysis?

Methods: We identified cases of rhabdomyolysis from the American College of Medical Toxicology's (ACMT) Toxicology Investigators Consortium (ToxIC). Cases were identified and reported by 50 medical toxicology consultative services throughout the United States and Canada. We report the toxicants that are associated with rhabdomyolysis and their epidemiologic and clinical characteristics. Within the "nervous system symptoms" data field, cases were divided into those with agitation ("agitation","rigidity","clonus","seizures") and sedation ("CNS depression" \& no agitation symptoms). The means of nominal variables were compared using Chi Square and Fisher exact test when data was sparse. Results: Between 2014-2020, there were 1812 cases of rhabdomyolysis with a known toxicant source. Most cases were adults (19-65 years) $(82 \%)$ and male (67\%). Most common agents associated with rhabdomyolysis were sympathomimetics $(21 \%)$, opioids $(17 \%)$, antidepressants $(8.5 \%)$ and sedatives/hypnotics $(7.1 \%)$. Most common laboratory findings included hyperlactatemia (32\%), troponinemia (30\%), AKI (25\%), metabolic acidosis. A very small percentage developed hyperkalemia > 
$6.0 \mathrm{mmol} / \mathrm{L}(.66 \%)$, Overall mortality in patients with toxicant associated rhabdomyolysis is $4.1 \%$. Sedated patients were more likely to develop AKI ( $45 \%$ vs. $7 \%$; p < 0.01), hypotension ( $30 \%$ vs. $10 \%$; $<<0.01)$, metabolic acidosis $(28 \%$ vs. $13 \%$; p < 0.01$)$, and had a higher mortality $(8.1 \%$ vs. $2.2 \% ; \mathrm{p}<0.01)$ than those with agitation.

Conclusion: Toxicant induced rhabdomyolysis is associated most commonly with sympathomimetics and opioids. Patients with sedativeassociated rhabdomyolysis had higher rates of critical illness, organ failure, and mortality.

\section{ToxIC}

This research was performed in collaboration with the ACMT Toxicology Investigators Consortium.

022. Burden of Adverse Events Related to the Treatment of COVID19 by Race/Ethnicity

Travis D Olives ${ }^{1,2}$, Audrey Hendrickson ${ }^{1}$, Alison Meyn ${ }^{3}$, Sharan Campleman ${ }^{3}$, Stephanie Abston ${ }^{3}$, Paul Wax ${ }^{3,4}$, Jeffrey Brent ${ }^{5}$, Kim $\mathrm{Aldy}^{3,4}$, On Behalf of the Toxicology Investigators Consortium (ToxIC) FACT Study Group

${ }^{1}$ Hennepin Healthcare, Minneapolis, MN, USA. ${ }^{2}$ Minnesota Poison Control System, Minneapolis, MN, USA. ${ }^{3}$ American College of Medical Toxicology, Phoenix, AZ, USA. ${ }^{4}$ University of Texas Southwestern Medical Center, Dallas, TX, USA. ${ }^{5}$ University of Colorado School of Medicine, Aurora, CO, USA

Background: COVID-19 has been associated with significant racial and ethnic disparities but reporting of COVID-19 treatment-related adverse drug events (TRADEs) by race/ethnicity remains limited.

Research Question: Describe the race and ethnicity of patients with COVID-19 TRADEs.

Methods: The FDA ACMT COVID-19 ToxIC (FACT) Pharmacovigilance Project is a multi-center active surveillance and reporting system identifying TRADEs to COVID-19 therapeutics. Fifteen participating sites reported cases identified through principal investigator and trained research assistant led chart reviews, and/or notification by healthcare teams and pharmacists. Data entered includes: demographics, case narratives, exposure details, clinical signs/symptoms, treatment, and outcomes of TRADEs. We reviewed all cases and stratified by race, highlighting remdesivir-associated acute kidney injury (AKI) and hepatic injury.

Results: Between 11/23/2020-11/9/2021, 764 TRADEs were identified across 15 sites. Overall, these occurred in: Non-Hispanic White $41.4 \%$ (n = 316); Hispanic/Latina/o 20\% ( $\mathrm{n}=153)$; Black/African American 19\% $(\mathrm{n}=145)$; Asian 3\% $(\mathrm{n}=23)$; American Indian/Alaskan Native $0.7 \%(\mathrm{n}$ = 5); Native Hawaiian/Pacific Islander 0.1\% ( $\mathrm{n}=1)$; and unknown/ uncertain race $15.8 \%(\mathrm{n}=121)$. Remdesivir-associated hepatic injury was reported in: Non-Hispanic White $22.3 \%(n=49)$; Hispanic/Latina/ o $15.5 \%$ ( $\mathrm{n}=34)$; Black/African American 10.5\% ( $\mathrm{n}=23)$; Asian $3.2 \%$ $(\mathrm{n}=7)$; American Indian/Alaskan Native $0.5 \%(\mathrm{n}=1)$; and unknown/ uncertain $0.9 \%(n=2)$. Remdesivir-associated AKI was reported in: NonHispanic White 39.3\% (n=11); Black/African American 32.1\% (n=9); Hispanic/Latina/o 21.4\% ( $\mathrm{n}=6$ ); Asian 7.1\% $(\mathrm{n}=2)$.

Conclusion: TRADEs were most commonly reported among NonHispanic White patients (almost half of all reported cases). Among Black/African American patients, however, remdesivir-associated AKI was three times more likely than hepatic injury, suggesting that remdesivir TRADEs may vary by race/ethnicity. Limitations include reporting bias, low numbers, site variability, and incomplete capture of sociocultural variables. These data suggest that reporting of COVID TRADEs varies by ethnic and racial backgrounds, while identifying a need for further investigation.

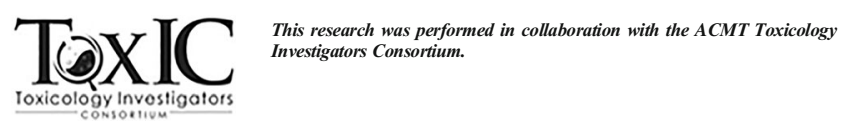

023. Drug Shortages Impacting Lead Chelators

Pelayia Soto $^{1,2}$, Kelly Johnson-Arbor ${ }^{1,3}$, Erin R Fox ${ }^{4}$, Maryann MazerAmirshahi $^{5}$

${ }^{1}$ National Capital Poison Center, Washington, DC, USA. ${ }^{2}$ George Washington University, Department of Emergency Medicine, Washington, DC, USA. ${ }^{3}$ MedStar Georgetown University Hospital, Department of Plastic and Reconstructive Surgery, Washington, DC, USA. ${ }^{4}$ University of Utah School of Medicine, Salt Lake City, UT, USA. ${ }^{5}$ MedStar Washington Hospital Center, Department of Emergency Medicine, Washington, DC, USA

Background: Prescription drug shortages are a long-standing public health problem that have impacted many antidotes. When multiple agents in the same therapeutic class are affected, patient care may be compromised.

Research Question: To describe drug shortages affecting lead chelators. Methods: Drug shortage data from January 2006 through September 2021 were obtained from the University of Utah Drug Information Service. We examined shortages for drugs used to treat lead toxicity including: succimer, dimercaprol, calcium disodium versenate, and penicillamine. Shortage data were analyzed focusing on the shortage duration, formulation, shortage reason and if the drug was a single source product (produced by one manufacturer). We also evaluated if multiple chelators were impacted by shortages at the same time.

Results: During the study period, there were 12 chelator shortages. The chelating agent involved in the most shortages was dimercaprol (four), followed by calcium disodium versenate and penicillamine (three each). At the end of the study period, all but two shortages (succimer 7.3 months and calcium disodium versenate 9.4 months) were resolved. Of resolved shortages, the median duration was 5.7 months (range 0.6-22.2 monthsthe longest was for penicillamine). Parenteral drugs were more commonly involved in shortages than oral agents (7/12). All 12 shortages were for single source drugs. There were 17 months during the study period that two or more chelators were in shortage. In most cases (7/12), the reason for shortage was not reported. For those that did provide a reason, production delays and manufacturing problems (two each) were the most common.

Conclusion: Drug shortages affected all of the major chelators used to treat lead toxicity over the 15 year study period, which can have significant implications for patient care. Future initiatives should focus on creating a sustainable drug supply and researching alternative chelator regimens to ensure adequate therapy for poisoned patients.

024. Drug Shortages Impacting Agents for Anticholinergic Delirium Pelayia Soto $^{1,2}$, Kelly Johnson-Arbor ${ }^{3}$, Erin R Fox ${ }^{4}$, Maryann MazerAmirshahi ${ }^{5}$

${ }^{1}$ National Capital Poison Center, Washington, DC, USA. ${ }^{2}$ George Washington University, Department of Emergency Medicine, Washington, DC, USA. ${ }^{3}$ MedStar Georgetown University Hospital, Department of Plastic and Reconstructive Surgery, Washington, DC, USA. ${ }^{4}$ University of Utah School of Medicine, Salt Lake City, UT, USA. ${ }^{5}$ MedStar Washington Hospital Center, Department of Emergency Medicine, Washington, DC, USA

Background: Anticholinergic delirium is associated with significant morbidity and mortality if not properly treated; yet many antidotes have been impacted by prescription drug shortages. 
Research question: To describe shortages affecting drugs used to treat anticholinergic delirium.

Methods: Shortage data from January 2001 through September 2021 were obtained from the University of Utah Drug Information Service. We examined shortages for first-line drugs used to treat anticholinergic delirium (physostigmine and parenteral benzodiazepines). We also evaluated second-line agents, including centrally-acting oral acetylcholinesterase inhibitors and dexmedetomidine. Patch formulations and peripherally acting-agents were excluded. Data analyzed included shortage duration, formulation, shortage reason, single source status (produced by one manufacturer), and if shortages overlapped.

Results: During the study period, there were 25 shortages impacting drugs used to treat anticholinergic delirium. There were six shortages of physostigmine, five of which were resolved. The median shortage duration was 5.1 months (range 4.4-8.8). There were 12 parenteral benzodiazepine shortages, with a median shortage duration of 16.6 months (1.688.3), and two active shortages at the end of the study period. The most commonly impacted benzodiazepine was diazepam (six shortages) followed by midazolam and lorazepam (three each). Dexmedetomidine was on shortage seven times, with a median duration of 7.1 months (0.418.8 ) and one active shortage remaining. There were no shortages for oral cholinesterase inhibitors. Physostigmine was the only single source drug involved. There were 78.3 months that two or more agents were in shortage. In many cases (12/25), the reason for shortage was not reported. When reported, manufacturing problems (eight) and supply/demand mismatch (four) were the most common reasons.

Conclusion: Shortages impacted the majority of agents used to treat anticholinergic delirium, which can have significant implications for patient care. Future initiatives should focus on creating a sustainable drug supply and researching alternative regimens to treat this potentially lifethreatening condition.

025. Radiation Injury Treatment Network Centers and Their Proximity to Medical Toxicology Resources

Mark Layer ${ }^{1}$, Girgis Fahmy ${ }^{2}$, Aaron Frey ${ }^{3}$, Ziad Kazzi ${ }^{2,4}$, Michael Kosnett $^{5}$, Emily Kiernan ${ }^{2,4}$

${ }^{1} . .{ }^{2}$ Emory University, Atlanta, GA, USA. ${ }^{3}$ University of Virginia, Charlottesville, VA, USA ${ }^{4}$ Georgia Poison Center, Atlanta, GA, USA

${ }^{5}$ University of Colorado, Denver, CO, USA

Background: The Radiation Injury Treatment Network (RITN) is a national organization of over 70 hospitals that aims to prepare for and respond to mass-casualty radiation emergencies, generally organized around bone marrow transplant centers and hematology/oncology departments to clinically manage radiation injuries. Medical toxicologists have expertise in management of radiation-related injuries and could provide valuable collaboration with local RITN centers. A previous study by Davlantes et al examined the proximity of RITN centers to medical toxicology resources, which we aim to reevaluate with current data and utilize to leverage further relationships.

Research Question: What is the current state of proximity between RITN transplant centers and medical toxicology resources?

Methods: This is a descriptive cross-sectional study. A listing of current RITN bone marrow transplant centers by city and state was created. Their proximity to medical toxicology resources was assessed categorically by center, city, and state. Resources include medical toxicology fellowship programs, inpatient toxicology services, outpatient toxicology clinics, and poison control centers. Data were derived from publicly available internet sources in November 2021.

Results: Seventy-five RITN bone marrow transplant centers were identified, compared to 59 previously. Thirty-one (41\%) have either a medical toxicology fellowship, inpatient toxicology service, or outpatient toxicology clinic within the same institution. Fifty-six $(75 \%)$ have at least one of these resources in the same city, compared to $57 \%$ previously. Fifty-nine $(79 \%)$ have at least one of these resources or a poison control center within the same city, increased from $73 \%$. Seventy-two (96\%) have at least one major medical toxicology resource within the state, increased from $95 \%$.

Conclusion: The majority of RITN transplant centers have at least one major medical toxicology resource within the same city, even as the network expands. Formal relationships between RITN centers and medical toxicologists would provide a valuable opportunity for collaboration and should be established.

026. Adverse Events Associated with Single Substance Accidental Ingestions of Anti-Xa Medications in Pediatric Patients

Vincent J Calleo $^{1,2}$, Syed Adnan ${ }^{1}$, Michael J Hodgman ${ }^{1,2}$

${ }^{1}$ SUNY Upstate Medical University, Syracuse, NY, USA. ${ }^{2}$ Upstate New York Poison Center, Syracuse, NY, USA

Background: The use of anti-Xa medications has increased over the last decade. Accidental exposures in young children are a concern and may result in health care facility (HCF) referrals. More data is needed to aid in appropriately managing these exposures. The objectives of this study were to investigate referral patterns, clinical outcomes, and treatment modalities using data from the National Poison Data System (NPDS) for children $\leq$ five years who have an accidental single substance ingestion of anti-Xa medications.

Methods: This is an IRB exempt retrospective review of information gathered from the NPDS from 2011-2019 for accidental single drug exposure to rivaroxaban, apixaban, and edoxaban. NPDS outcome codes (no effect, minor effect, moderate effect, major effect, and death) were used. Clinical effects and therapeutic treatments were tabulated. Descriptive statistics were used.

Results: Seven hundred forty four cases met inclusion criteria. Three hundred fifty cases were managed at home, with 346 having no effect and four having minor effects. Of the 394 managed at a HCF, 337 had no effect, 33 had minor effects, 22 had moderate effects, and two had major effects. Clinical effects recorded were mostly 'increased PT/INR' and 'coagulopathy-other' though no significant bleeding was reported. One moderate outcome was coded 'asystole cardiac arrest' without any interventions. Major outcomes were 'miscellaneous-other' and 'PT/INR prolonged; coagulopathy-other'. Six hundred twenty-six patients received no activated charcoal and only one of these patients had a major outcome which only required 'fluids' and 'food/snack'.

Conclusion: Accidental ingestion of single substance anti-Xa medications rarely results in significant morbidity in pediatric patients. Given this population's low incidence of clinically significant effects after accidental ingestion of these substances (many without activated charcoal administration), it may be reasonable to manage these patients at home. Currently over half these exposures are managed at home by Poison Centers and this data supports this practice.

027. Evaluation of the QT Nomogram in Poisoned Pediatric Patients Caleb Fredrickson, David B Liss, Michael E Mullins Washington University, Saint Louis, MO, USA

Background: Many xenobiotics antagonize the inward rectifier potassium channel (Ikr), leading to QT prolongation on ECG with increased risk for ventricular dysrhythmia. The QT nomogram outperforms QT correction by Bazett's formula (QTcB) for the prediction of dysrhythmia in adults.

Hypothesis: We propose the QT nomogram is a safe tool to assess risk of ventricular dysrhythmia in a poisoned pediatric population and will achieve fewer false positives while achieving equal or greater sensitivity than QTcB.

Methods: We performed a retrospective chart review of pediatric patients presenting to a single emergency department (ED) from June 2018 to June 2021. We included patients diagnosed by the attending toxicologist with poisoning by any xenobiotic and with an ECG obtained within four hours of presentation. We excluded patients with congenital heart disease or transplant. We reviewed each ECG and recorded QT, QTcB, and heart 
rate. We compared QTcB thresholds of $440 \mathrm{~ms}$ and $500 \mathrm{~ms}$ to the QT-HR pair plotted on the QT nomogram. We reviewed charts for provider notes, ECGs, nursing notes, and rhythm strips to detect instances of ventricular dysrhythmia.

Results: We reviewed 68 charts, with six excluded (ECG unavailable 1, ECG $>4$ hours after ED arrival 2, congenital heart disease 1, nontoxicology patient 1 , tachycardia beyond QT nomogram 1). Twentyfive $(40 \%)$ were "high risk" by QTcB $>440 \mathrm{~ms}$, vs. four $(6 \%)$ by the nomogram ( $\mathrm{p}<0.001)$. One ECG was "high risk" by QTcB $>500 \mathrm{~ms}$ and by QT nomogram. No patient had a recorded ventricular dysrhythmia.

Conclusions: In this small, retrospective pilot study, the QT nomogram had fewer false positives compared to a QTcB cutoff of $440 \mathrm{~ms}$. Using the QT nomogram instead of QTcB threshold of $440 \mathrm{~ms}$ would allow onethird of our patients to safely forgo cardiac monitoring. Since no dysrhythmia events were recorded, sensitivity cannot be assessed.

028. Time for Change: Pediatric Coin Ingestion Statistics from a Single Poison Center

Vincent $\mathrm{J}_{\text {Calleo }}{ }^{1,2}$, Cory Howard ${ }^{1,2}$, Daniel Tirado ${ }^{1}$, Michael J Hodgman $^{1,2}$

${ }^{1}$ SUNY Upstate Medical University, Syracuse, NY, USA. ${ }^{2}$ Upstate New York Poison Center, Syracuse, NY, USA

Background: Accidental coin ingestions are common in young children. In 2019, 3002 total cases of coin ingestions were reported nationally, with 32 cases having moderate or major outcomes. Informal polling of Poison Specialists at our Poison Center (PC) revealed disparate practice patterns managing these exposures. The objectives for our study are to: 1) investigate our PC's practice patterns, 2) determine the number of pediatric patients who develop symptoms and 3) document the number of supradiaphragmatic coins after accidental ingestions.

Methods: This is an IRB exempt 10-year retrospective review of cases at the Upstate New York Poison Center (UNYPC). Toxicall search criteria were unintentional ingestion of any coin in children ages $0-5$ years. Cases from outside the UNYPC catchment area and asymptomatic patients greater than 48 hours post-ingestion were excluded. Data was analyzed using descriptive statistics.

Results: Of the 347 calls to the PC, 333 were referred to, in route to, or at a healthcare facility (HCF) at the time of call. One hundred ninety-nine cases did not have PC follow up or were not known to have gone to a HCF. Of the 134 cases with known HCF evaluation, 131 had X-rays performed, with a coin present in 103. Six of $103(5.8 \%)$ showed a coin above the diaphragm; four of these six $(66.7 \%)$ did not have symptoms in the ED. Of the 97 cases with coins below the diaphragm, one of 97 (1\%) had symptoms in the ED.

Conclusion: Young children with supradiaphargmatic coins may be asymptomatic at ED evaluation. Practice patterns by our Poison Specialists were highly variable and may contribute to a low case follow up. A limitation of our finding is the lack of a standardized definition of an asymptomatic child. A more uniform approach to these children by our $\mathrm{PC}$ is warranted as is further investigation.

029. Adaptation of Toxicology Billing to Reflect the Pressures of the COVID-19 Pandemic

Timothy J Wiegand, Peter W Crane

University of Rochester Medical Center, Rochester, NY, USA

Background: Demonstration of value, including generation of revenue, is a necessity for a toxicology service to remain sustainable as a hospital service line. We previously reported our billing and reimbursement at a large urban academic institution pre-covid and have demonstrated that a toxicology service can have sustainability and growth. The pandemic has mandated changes in billing practices that we suspect are widespread throughout the country. In this work, we describe our experiences with toxicology billing and reimbursement during the summer and fall of 2020, mid-pandemic.
Hypothesis: Toxicology billing changed to reflect the impact of the COVID-19 pandemic including increasing use of telemedicine.

Methods: Convenience sample of all charges and reimbursement for June 2020-November 2020. The data was derived from QA and standard billing databases for our home institution. No identifiable patient information was collected.

Results: A total of $\$ 634,349$ charges were billed during this time. Of this, $\$ 177,576$ was collected for a total collection rate of $27.79 \%$. Payments recorded are on a rolling basis, and there was a significant lag from typical time to reimbursement related to covid-19. Average monthly billing was $\$ 105,724$ and average payment/reimbursement was $\$ 28,336$. Due to covid, all office visits were conducted and billed as telehealth visits instead of in person. Average charge per CPT code was $\$ 417.14$ with average payment per CPT was $\$ 114.26$.

Conclusion: The covid pandemic disrupted medicine on many levels and has driven substantial changes in billing and reimbursement. In our practice a substantial portion of services were subsequently provided through various forms of telehealth. We previously reported our billing data for a full fiscal year with over $\$ 600,000$ in charges and $\$ 177,059$ in total reimbursement. Despite challenges associated with the pandemic our six-month billing during this time suggests that toxicology services are sustainable, and may even support growth, particularly with increasing reliance on telehealth.

030. Board Certification Trends in Medical Toxicology Sefira B Fialkoff ${ }^{1}$, Paul Wax ${ }^{1}$, Lewis Nelson ${ }^{2}$

${ }^{1}$ American College of Medical Toxicology, Phoenix, AZ, USA. ${ }^{2}$ Rutgers New Jersey Medical School, Newark, NJ, USA

Background: In 1994 the American Board of Medical Specialties (ABMS) officially recognized medical toxicology (MT) as a sub-specialty. A certification examination was developed by a sub-board of experts representing the three sponsoring boards: the American Boards of Emergency Medicine, Pediatrics, and Preventive Medicine. These sponsoring boards reflected the heterogeneity of those with MT expertise.

Research Question: What are the numerical trends of new diplomates board certified (BC) over time, and what are their primary board statuses? Methods: Data was obtained from ABMS on all BC MTs since 1994. The number of candidates who became certified with each two-year examination cycle and the primary board status of new diplomates were analyzed.

Results: From 1994-2020, 672 physicians gained BC in MT by ABMS, 555 of whom have active certificates as of 2021. The largest cohort was in 1994 when 67 were certified. Since the inception of required fellowship training in 2000, newly certified medical toxicologists ranged from 33 (2000) to 61 (2020). From 2010-2020, the mean of newly BC medical toxicologists was 52 every two years (26 per year). Regarding primary specialty, from $1994-2000,59 \%$ of the new diplomates were only primarily boarded in emergency medicine (EM). In the 2008-2010 cohorts $85 \%$ were primarily board certified in EM only, whereas in 2020 being board certified in only EM increased to $97 \%$.

Conclusions: In recent years an average of 52 physicians have become $\mathrm{BC}$ in MT with each two-year exam cycle. The percentage of those whose primary training was only EM was at its highest point (97\%) during the last exam cycle, continuing a trend that has been apparent for many years. To regain the heterogeneity of medical toxicologists' primary training, if desired, a concerted effort to understand the value of primary board diversity and these demographic shifts, will be required to attract fellow candidates from outside EM.

031. Antivenom Supply and Demand: An Analysis of Antivenom Availability and Utilization in South Africa

Natalie R Neumann ${ }^{1,2}$, Arina du Plessis ${ }^{3}$, Daniël Van Hoving ${ }^{4}$, Christopher O Hoyte ${ }^{2,5}$, Anné Lermer ${ }^{3}$, Carine Marks ${ }^{3}$

${ }^{1}$ Department of Emergency Medicine, Yale University School of Medicine, New Haven, CT, USA. ${ }^{2}$ Rocky Mountain Poison and Drug 
Safety, Denver, CO, USA. ${ }^{3}$ Division of Clinical Pharmacology, Faculty of Medicine and Health Sciences, Stellenbosch University, Cape Town, Western Cape, South Africa. ${ }^{4}$ Division of Emergency Medicine, Stellenbosch University, Cape Town, Western Cape, South Africa. ${ }^{5}$ Department of Emergency Medicine, University of Colorado Anschutz Medical Campus, Aurora, CO, USA

Background: Snakebites are a neglected tropical disease. In many areas, specific envenomation incidence and antivenom administration rates are unknown. This study compared antivenom availability to envenomation rates in South Africa.

Methods: This is a retrospective study. We identified, extracted, and reviewed all cases of envenomation reported to the Poison Information Helpline of the Western Cape of South Africa (PIHWC) from June 1, 2015 to May 31, 2020 by public hospitals in the Western Cape. We also administered a standardized interview to pharmacies of the 40 hospitals. We used descriptive analysis to compare envenomation rates and PIHWC recommendations to administer antivenom, to antivenom stock and expiration.

Results: Public hospitals reported 300 envenomations, 122 from snakes including 90 envenomations from hospitals outside Cape Town (CT). The PIHWC recommended anti-venom administration in $26 \%$ of cases $(n=32)$. All hospital pharmacies queried, answered our questions regarding antivenom stocking and availability. Of pharmacies queried, $60 \%$ had a stocking strategy for anti-venom; $24 \%$ had a standard operating procedure for envenomated patients. At the level of the district hospital, we found that CT hospitals had higher ratios of antivenom vials to annualized envenomations and recommendation for antivenom administration than their non-CT counterparts ( 33 vs. $16 ; 365$ vs. 73 ). When hospitals were grouped into referral networks, urban hospitals still had more antivenom than their rural counterparts at the level of regional hospitals. There was no difference at the tertiary level. One regional referral network had fourfold more antivenom per envenomation than the others. Most referral networks stocked nearly two years' worth of antivenom. One regional network had stock which would expire prior to utilization.

Conclusion: This data describes antivenom supply and demand in Africa. It suggests South African urban hospitals have a relative excess of antivenom compared to rural counterparts up to the level of the regional network.

032. Methods of Tobacco Harm Reduction among Smokers in Malawi: Case Study of Chitipa, Ntcheu and Balaka Districts Vita Mithi ${ }^{1,2}$, Rebecca S. Dewey ${ }^{3}$

${ }^{1}$ Armref Data for Action in Public Health Research, Mzuzu, North, Malawi. ${ }^{2}$ MESA Alliance, hosted by: ISGlobal, Barcelona, Catalonia, Spain. ${ }^{3}$ University of Nottingham, Nottingham, United Kingdom

Significance: The aim of the study was to investigate the methods of tobacco harm reduction products used by smokers in Malawi, in an attempt to reduce tobacco harm.

Method: The study employed a cross-sectional survey, with both quantitative and qualitative data collection, in three districts, one from each region across Malawi. Participants were recruited using the technique of snowball sampling. One thousand two hundred fifty-eight current and exsmokers were recruited, age range 15-55 years. The sample size for each district was calculated using the standard formula with a $95 \%$ confidence interval or significance of $\mathrm{p}=0.05$.

Results: Tobacco harm reduction products, also known as safer nicotine products, are not widely available in Malawi; as such their use is not statistically significant at $5 \%$. However, the following resources were used to reduce tobacco harm: fruits like Malambe (Baobab), Banana, Masau (Jujube), Mtera (local herbs), sweets, chewing gum, fried maize and milk. Solutions: cigarette filters mixed with water, a mixture of pig milk, tobacco and drinking water. In addition, ngirimbo and chingambwe were used in a smokeless way to reduce tobacco harm.
Conclusion: Across Malawi, smokers use local tobacco harm reduction products compared to safer nicotine products. This is compounded by the absence of information on safer nicotine products, a lack of service providers, poverty and use of local smokeless tobacco.

033. Retrospective Case Series of Hallucinogenic Mushrooms in Northeast Turkey

Girgis S Fahmy ${ }^{1}$, Vildan Ozer ${ }^{2}$, Ziad Kazzi ${ }^{1}$, Aleyna Arslan², Aynur Sahin $^{2}$

${ }^{1}$ Emory University, Atlanta, GA, USA. ${ }^{2}$ Karadeniz Technical University Faculty of Medicine, Trabzon, Turkey

Background: Hallucinogenic mushrooms that contain psilocybin or ibotenic acid grow in Europe, South America, Mexico, and the United States. This case series aims to describe the clinical manifestations of intoxication following the ingestion of hallucinogenic mushrooms in Northeast Turkey and to raise awareness of the hazards associated with ingesting foraged mushrooms due to misidentification.

Hypothesis: The cluster of illness in Northeast Turkey was due to the ingestion of similar mushrooms with hallucinogenic properties.

Methods: This is a retrospective case series of hallucinogenic mushroom ingestions presenting to a tertiary care hospital in Northeast Turkey over a four month period between June 1 and September 30, 2021. Data was acquired through the electronic medical record of a medical toxicology unit at Farabi Hospital in Trabzon, Turkey.

Results: Fourteen patients presented after ingesting mushrooms during the study period. All ingestions occurred due to misidentification. The mean age was $55.7 \pm 13.1$ years. Fifty seven percent of patients were women. The onset of vomiting was within one to two hours of ingestion in all patients who vomited. The most common presenting symptoms were nausea/vomiting $(71 \%, \mathrm{n}=10)$, somnolence $(42.9 \%, \mathrm{n}=6)$, and hallucinations $(35.7 \%, \mathrm{n}=5)$. The mean systolic blood pressure was $135 \mathrm{~mm} \mathrm{Hg}$ (IQR: 120-150). The mean time to admission into the hospital was six hours (IQR: 4-8.2). The mean time of symptom resolution was 12 hours (IQR: 12-13.5). Some patients required benzodiazepines. Comprehensive metabolic panels were unremarkable in all patients.

Conclusion: Although the mushrooms in our cluster of illness were not identified, it was possible to manage them using a toxidrome based risk assessment and supportive care. The patients were all managed with supportive care for up to 24 hours. Proper identification without the assistance of a mycologist is difficult, which makes the ingestion of foraged mushrooms hazardous to health.

034. Toxicology of Ngirimbo: Analysis to Determine the Levels of Trace and Heavy Metals, Moisture, Nicotine, pH and Microbial Assessment

Vita Mithi ${ }^{1,2}$, Happy Matchabani Mhango ${ }^{3}$, Getrude Boti Phiri ${ }^{3}$, Rebecca S. Dewey ${ }^{4}$

${ }^{1}$ Armref Data for Action in Public Health Research, Mzuzu, North, Malawi. ${ }^{2}$ MESA Alliance, hosted by: ISGlobal, Barcelona, Catalonia, Spain. ${ }^{3}$ Malawi Bureau of Standards, Blantyre, Malawi. ${ }^{4}$ University of Nottingham., Nottingham, United Kingdom

Background: Tobacco and other similar/related products represent a substantial proportion of recreationally used substances in Malawi. Ngirimbo is a smokeless substance taken orally to reduce tobacco smoking. This study aimed to determine the toxicological characteristics of Ngirimbo in terms of pesticide residues, microbial, trace and heavy metal contaminants, herbal compounds, and nicotine, moisture, and $\mathrm{pH}$ levels.

Methods: Samples were analyzed using atomic absorption spectrophotometry, titration, X-ray fluorescence spectroscopy, UV-visible Spectrophotometer, gas chromatography-mass spectrometry, $\mathrm{pH}$ and gravimetric analysis, ISO4833, ISO21527, ISO16654, and ISO6579.

Results: From a total of 12 samples, five samples contained nicotine ranging from $0.08 \%$ to $0.84 \%$, while the remaining seven samples 
showed no trace of nicotine. Sample pH ranged from 6.68 to 8.32 , and moisture content from $12.87 \%$ to $47.11 \%$. Samples had no detectable contamination with chlorine or pesticide residue. Heavy metals tested: Cadmium, Nickel, Lead, and Copper were found to be below the detection limit of $0.01 \mathrm{mg} / \mathrm{kg}$. On the other hand, X-ray fluorescence spectroscopy analysis revealed the presence of Nickel, Bromine, Rubidium, Strontium, Zirconium, Molybdenum, Rhodium, Cadmium, Chromium, and Tellurium. Samples had good levels of Calcium, Iron, Potassium, Sodium, and Zinc ranging from $23 \mathrm{mg} / \mathrm{kg}$ to $57,800 \mathrm{mg} / \mathrm{kg}$. Samples contained phytocompounds and herbal material of medicinal relevance. In terms of the microbiological content, Escherichia coli and Salmonella were absent, while Moulds and Yeast were present at a level of $<1 \mathrm{cfu} / \mathrm{g}$, with the total plate count varying across all samples between $1,400 \mathrm{cfu} / \mathrm{g}$ and $640,000 \mathrm{cfu} / \mathrm{g}$.

Conclusion: These findings demonstrate that the current state of Ngirimbo available in Malawi is toxic and a hazard to human health. Prolonged use of Ngirimbo may lead to users developing mouth cancers or thermal burns to the oral mucosal membrane. Further, Ngirimbo consumers are at risk of developing dental caries, known to harbor microorganisms and development of infectious diseases.

035. Analysis of Arsenic in Tobacco of Cigarettes Sold in Indian Market using Atomic Absorption Spectrophotometer with Vapour Generator Assembly

Rohit Kanojia $^{1}$, Ashok Kumar Jaiswal ${ }^{2}$, Sunita Bhagat ${ }^{1}$, Neha Afaria ${ }^{2}$, Kapil Kumar ${ }^{2}$

${ }^{1}$ University of Delhi, Delhi, India. ${ }^{2}$ All India Institute of Medical Sciences, Delhi, India

Background: Arsenic is toxic to humans in every form and quantity. Inorganic forms of arsenic are usually more toxic than organic forms. Humans are exposed to arsenic via dermal exposure, directly consuming food containing arsenic, smoking or breathing air having arsenic vapors etc. Symptoms of arsenic acute poisoning can vary from recurring diarrhea, thickening and discoloration of skin, nausea, abnormal heartbeat, numbness in limbs, partial paralysis, occurring of small corns or warts on soles, torso and palms. Biological matrices like urine and blood are the most significant in assessing exposure levels of arsenic. Even after excretion, a small amount of arsenic is left in the skin and keratin-rich tissues like nails and hair. Ground water is the main source of arsenic contamination in flora. Vegetables, fruits and even tobacco has arsenic content due to the same. A large number of teenagers consume cigarettes despite a ban in selling and consuming tobacco products under the age of 18 years. Ultimately, getting exposed to smoke containing arsenic vapors, harming themselves as well as the passive smokers.

Hypothesis: Tobacco used in Indian cigarette brands contains arsenic in toxic levels.

Methods: This is an experimental study to analyze the amount of arsenic in tobacco sold in Indian markets. Atomic Absorption Spectrophotometer with Vapor Generator Assembly (AAS-VGA) is used for this study due to its high precision and simplicity to check a urine sample for presence of arsenic in it.

Results: A total of 14 Cigarettes' brands were taken for the study. Tobacco from almost all of the cigarettes contained arsenic at a toxic level.

Conclusion: A large number of people consuming tobacco through cigarettes' smoke and passive smokers are at risk of various lethal and serious complications caused by arsenic toxicity. AAS-VGA is a promising technique for arsenic analysis in plant-based samples.

036. Reddit Discussions about Buprenorphine Associated Precipitated Withdrawal in the Era of Fentanyl

Anthony Spadaro ${ }^{1}$, Abeed Sarker ${ }^{2}$, Whitney Hogg-Bremer ${ }^{2}$, Jennifer S Love $^{3}$, Nicole O'Donnell ${ }^{1}$, Lewis S Nelson ${ }^{4}$, Jeanmarie Perrone ${ }^{1}$

${ }^{1}$ Perelman School of Medicine at the University of Pennsylvania Department of Emergency Medicine, Philadelphia, PA, USA. ${ }^{2}$ School of Medicine at Emory University, Atlanta, GA, USA. ${ }^{3}$ Icahn School of Medicine at Mount Sinai, New York City, NY, USA. ${ }^{4}$ Rutgers University Department of Emergency Medicine, Newark, NJ, USA

Background: Buprenorphine is an evidence-based treatment for Opioid Use Disorder (OUD). Standard buprenorphine induction requires a period of opioid abstinence to minimize risk of precipitated opioid withdrawal (POW). Our objective was to study the impact of the increasing presence of fentanyl and its analogs in the opioid supply of the United States, on buprenorphine induction and POW, using social media data from Reddit. Research Question: What can we learn from Reddit discussions about the association of precipitated opioid withdrawal (POW), fentanyl, and self-management strategies for avoiding POW?

Methods: This is a data-driven, mixed methods study of opioid-related forums, called subreddits, on Reddit to analyze posts related to fentanyl, POW, and buprenorphine induction. The posts were collected from seven subreddits using an application programming interface for Reddit. We applied natural language processing to identify subsets of salient posts relevant to buprenorphine induction, and performed manual, qualitative, thematic analyses of them.

Results: Two hundred sixty-seven thousand one hundred thirty-six posts were retrieved from seven subreddits. Fentanyl mentions increased from three in 2013 to 3870 in 2020, and POW mentions increased from two (2012) to 332 (2020). Manual review of 384 POW-mentioning posts and 106 'Bernese method' (a microdosing induction strategy) mentioning posts revealed common themes and peoples' experiences. Specifically, presence of fentanyl caused POWs despite long abstinence durations, and alternative induction via microdosing were frequently recommended in peer-to-peer discussions.

Conclusion: This study found that increased social media chatter on Reddit about POW correlated with fentanyl mentions. A subset of posts described microdosing as a self-management strategy to avoid POW. Reddit posts suggest that people are utilizing these strategies to initiate buprenorphine due to challenges arising from fentanyl prevalence in the opioid supply.

037. Concurrent Psychiatric Illness Among Patients with Substance Abuse-related Emergencies

Simran Koura ${ }^{1}$, Joshua Masdon ${ }^{2}$, Avian White ${ }^{1}$, Kori Brewer ${ }^{2}$, William Meggs $^{2}$

${ }^{1}$ East Carolina University Brody School of Medicine, Greenville, NC, USA. ${ }^{2}$ Brody School of Medicine, Emergency Medicine, Greenville, $N C, U S A$

Background: Patients with substance abuse related emergencies often have concurrent psychiatric illnesses that compromise and modify appropriate evaluation, treatment, and disposition.

Research Question: Are patients presenting to a hospital for substance abuse-related emergencies more likely to have been diagnosed with a psychiatric illness?

Methods: A retrospective chart review was conducted with IRB approval. Three hundred and forty charts were randomly selected, of which 160 had an emergency related to an illicit drug and 180 had an alcohol-related emergency. Data collected included demographics, tobacco abuse, chief complaint, suicide attempt, psychiatric diagnoses, urine drug screen (barbiturates, benzodiazepines, cocaine, opiates, amphetamines, cannabinoids, phencyclidine, MDMA, and fentanyl) and ethanol results.

Results: Depression followed by schizoaffective disorder were the most commonly documented psychiatric illnesses amongst patients presenting with substance related emergencies and documented positive urine drug screens. Of the patients testing positive for ethanol with a psychiatric diagnosis, $36 \%$ had a history of depression and $27 \%$ had a history of schizoaffective disorder. Of those testing positive for cannabis with a psychiatric diagnosis, $40 \%$ had a history of depression and $25 \%$ had a history of schizoaffective disorder. Of those testing positive for opiates with a psychiatric diagnosis, $51 \%$ had a history of depression and $18 \%$ had a history of schizoaffective disorder. 
Conclusion: Depression was the most common psychiatric illness seen in patients presenting with substance abuse related emergencies. Persons presenting with substance abuse disorders can have psychiatric illnesses that can modulate evaluation, diagnosis, and treatment.

038. Co-presence of Mitragynine and Fentanyl in Emergency Department Patients with Suspected Opioid Overdose

Adrienne R Hughes ${ }^{1}$, Robert G Hendrickson ${ }^{1}$, Kim Aldy ${ }^{2,3}$, Sharan Campleman ${ }^{2}$, Alison Meyn ${ }^{2}$, Alex Krotulski ${ }^{4}$, Barry Logan ${ }^{4,5}$, Paul $\mathrm{Wax}^{2,3}$, Jeffery Brent ${ }^{6}$, Alex F Manini ${ }^{7}$, On Behalf of the Toxicology Investigators Consortium (ToxIC) Fentalog Study Group

${ }^{1}$ Oregon Health and Science University, Portland, OR, USA. ${ }^{2}$ American College of Medical Toxicology, Phoneix, AZ, USA. ${ }^{3}$ University of Texas Southwestern Medical Center, Dallas, TX, USA. ${ }^{4}$ Center for Forensic Science Research and Education at the Fredric Rieders Family Foundation, Willow Grove, PA, USA. ${ }^{5}$ NMS Labs, Horsham, PA, USA. ${ }^{6}$ University of Colorado School of Medicine, Aurora, CO, USA. ${ }^{7}$ Icahn School of Medicine at Mount Sinai, New York, NY, USA

Background: Kratom (Mitragyna speciosa) is an herbal drug that produces stimulant effects at low doses and opioid-receptor mediated effects at higher doses. Mitragynine is used as a marker for kratom exposure and is responsible for its clinical and psychoactive effects. Fentanyl plus kratom use is poorly described despite independently escalating prevalence of use recently. Research Question: To describe the clinical course of patients with coexposure to mitragynine and fentanyl in the ongoing ToxIC Fentalog Cohort Study.

Methods: This case series includes adult ED patients with co-exposure to mitragynine and fentanyl in the ongoing ToxIC Fentalog Cohort Study. Comprehensive toxicological testing was performed on residual blood samples via liquid chromatography quadrupole time-of flight mass spectrometry for the presence of over 900 psychoactive substances. Cases with mitragynine identified in biologic samples were reviewed.

Results: Between 10/20-10/21, > 400 biological samples of patients with suspected opioid overdose were analyzed from nine sites over eight states. Mitragynine was detected in seven samples from two states (Pennsylvania, Michigan). Ages ranged from $23-44$ and $71 \%$ were male. One patient developed hypotension ( $\mathrm{SBP}<80$ ), rhabdomyolysis (peak creatinine kinase $>275 \mathrm{~K}$ ), and acute kidney injury (peak Cr 5.58) requiring seven hemodialysis sessions. No patients required ventilatory support and there were no deaths. Five patients received prehospital naloxone. Response to naloxone for the first dose in three cases was improved respiratory rate and level of consciousness. Two cases had no initial response, but both received an additional dose of naloxone with improved respiratory rate and level of consciousness. Fentanyl was coidentified with mitragynine in all seven cases.

Conclusion: In our cohort, mitragynine was identified along with fentanyl in all cases, suggesting either addition to the illicit opioid supply or concomitant use. Combined illicit fentanyl and kratom use resulted in multisystem organ failure in one patient, but all survived the overdose.

\section{ToxIC}

This research was performed in collaboration with the ACMT Toxicology Investigators Consortium.

039. Trends in Fentanyl Detection in Emergency Department Patients With Opioid Related Diagnoses.

Sophia Faude ${ }^{1}$, Austin Kilaru ${ }^{1}$, Mucio Kit Delgado ${ }^{1}$, Anthony Spadaro ${ }^{1}$, Ashish Thakrar ${ }^{1}$, Lewis S Nelson ${ }^{2}$, Margaret Lowenstein ${ }^{1}$, Jeanmarie Perrone $^{1}$

${ }^{1}$ Perelman School of Medicine, University of Pennsylvania, Philadelphia, PA, USA. ${ }^{2}$ Rutgers New Jersey Medical School, Newark, NJ, USA
Background: As fentanyl has become increasingly prevalent in the illicit drug supply, fentanyl use has displaced other illicit opioids including oxycodone and heroin.

Research Question: What patient and clinical characteristics are associated with fentanyl detection in urine drug testing (UDT) of emergency department (ED) patients?

Methods: This is a retrospective review of a cohort of 4075 ED patients with opioid-related ICD-10 diagnosis codes and UDT collected at provider discretion from January 2020-March 2021. The primary outcome was presence of fentanyl on UDT stratified by time (quarterly). We used multivariable logistic regression to determine the likelihood of positive fentanyl tests by patient characteristics and time. We report adjusted odds ratios and adjusted probabilities by quarter. We also calculated the proportion of tests in which fentanyl was the only opioid detected.

Results: Four thousand seventy-five patients had a UDT collected (63.7\% male, mean age 43.7), and 1224 identified fentanyl. The likelihood that patients tested positive for fentanyl significantly increased over time (OR 1.09; 95\% CI $1.03-1.14$ ), after adjusting for patient age, sex, race/ethnicity, and clinical presentation. The adjusted probability of positive fentanyl tests increased by $1.4 \%$ per quarter, from $26.1 \%(95 \% \mathrm{CI}$ 23.7 - 28.5) to $32.8 \%$ (95\% CI 30.3 - 35.4). Patients with opioid withdrawal were significantly more likely to have fentanyl detected $(45.7 \%$ $(95 \%$ CI $41.7-49.6))$ than patients with opioid overdose $(31.9 \%(95 \% \mathrm{CI}$ 28.8 - 35.1). Fifty-eight percent of patients with fentanyl on UDT were negative for all other opioids.

Conclusions: The rate of fentanyl positive tests increased by nearly seven percent over the study period for patients in whom a UDT was collected; most fentanyl-positive tests did not reveal the presence of other opioids. Fentanyl was more likely to be detected in patients with withdrawal vs overdose diagnoses. Future prospective studies should confirm these trends and examine underlying reasons for these findings.

\section{Brorphine, Isotonitazene and Metonitazene Emerge as New Age} Opioids

Alexandra M Amaducci ${ }^{1}$, Kim Aldy ${ }^{2,3}$, Sharan Campleman ${ }^{2}$, Alison Meyn $^{2}$, Stephanie Abston ${ }^{2}$, Alex Krotulski ${ }^{4}$, Barry Logan ${ }^{4,5}$, Paul $\mathrm{Wax}^{2,3}$, Jeffrey Brent ${ }^{6}$, Alex F Manini ${ }^{7}$, On Behalf of the Toxicology Investigators Consortium (ToxIC) Fentalog Study Group

${ }^{1}$ Lehigh Valley Health Network-USF Morsani College of Medicine, Allentown, PA, USA. ${ }^{2}$ American College of Medical Toxicology, Phoenix, AZ, USA. ${ }^{3}$ University of Texas Southwestern Medical Center, Dallas, TX, USA. ${ }^{4}$ Center for Forensic Science Research and Education at the Fredric Rieders Family Foundation, Willow Grove, PA, USA. ${ }^{5}$ NMS Labs, Horsham, PA, USA. ${ }^{6}$ University of Colorado School of Medicine, Aurora, CO, USA. ${ }^{7}$ Icahn School of Medicine at Mount Sinai, New York, NY, USA

Background: New Age Opioids (NAOs) are classified as new nonfentanyl opioids in the illicit opioid supply. Brorphine, isotonitazene and metonitazene are NAOs with a piperidine benzimidazolone structure. Research Question: We report six cases of opioid overdose found to contain NAOs. Methods: This case series is from the Toxicology Investigators Consortium (ToxIC) Fentalog Study Group, an ongoing multicenter study across the United States. Consecutive ED patients with a presumed acute opioid overdose and residual blood samples were included. Exclusion criteria were: age $<18$, prisoners, and those with nontoxicologic diagnoses. Information collected by chart review included demographics, past medical and substance use history, clinical course, vital signs, and disposition. Discarded blood samples for all included patients were sent for toxicologic confirmation performed by liquid chromatography quadrupole time-of-flight mass spectrometry for the presence of over 900 substances including novel psychoactive substances and their metabolites.

Results: Between 10/6/20-10/31/21, 412 cases met inclusion criteria, with six positive for brorphine, isotonitazene and metonitazene. Age range was 20-57. The majority were female $(n=4)$. All patients presented 
with an opioid toxidrome and received naloxone. All samples contained fentanyl and a stimulant. In the two brorphine cases, they reported using a parental opioid such as heroin or fentanyl. Both had a depressed level of consciousness (DLOC) and respiratory depression which were reversed with up to two naloxone doses. Isotonitazene was detected in two patients; both had DLOC and received two naloxone doses. The two patients that were found to have metonitazene presented in cardiac arrest. One patient died despite six mg of naloxone and the other survived after receiving $10 \mathrm{mg}$ of naloxone.

Conclusion: NAOs may confound a typical opioid overdose presentation and might require higher than typical naloxone dosing. Both metonitazene patients had cardiac arrests, and other NAOs had decreased response to naloxone likely due to high potency.

\section{Ioxicology Invostigators $\begin{aligned} & \text { This research was performed in collaboration with the ACMT Toxicology } \\ & \text { Investigators Consortium. }\end{aligned}$}

Posters, Abstracts 041-147

041. Danger South of the Border: Severe Cardiotoxicity and Death After Ibogaine Administration

Shaun Carstairs

Scripps Healthcare, San Diego, CA, USA. 98point6 Inc., Seattle, WA, USA

Background: Ibogaine is a naturally occurring psychoactive compound derived from the root of the Tabernanthe iboga plant. Although its use is illegal in the United States, it is used in other countries (including Mexico) for treatment of drug and alcohol addiction, and many U.S. residents visit these clinics for treatment with ibogaine. Ibogaine has been reported to have significant cardiotoxicity, but experience with this substance in the U.S. is limited; we report two fatal cases of ibogaine toxicity in patients transported to the U.S. for treatment.

Methods: This is a two-patient chart review. Patient one: a 27-year-old female, was attending a detoxification facility in Baja California (B.C.), Mexico for treatment of benzodiazepine addiction. She was administered an unknown amount of ibogaine intravenously (IV), and shortly thereafter became unresponsive and suffered convulsions. She was intubated and transported to a hospital in the U.S. (approximately 30 minutes from the Mexican facility). Patient two: a 33-year-old male, was attending a different detoxification facility in B.C. for treatment of alcohol and heroin addiction. He was administered an unknown amount of ibogaine IV and became unresponsive and suffered convulsions. He was intubated and transported to the same hospital in the U.S.

Results: Patient one was noted on initial EKG to have a QTc of $602 \mathrm{~ms}$. Patient two was noted on initial EKG to have a QTc of $649 \mathrm{~ms}$. Initial workup, including labs and head CT, was unremarkable for both patients. Both suffered repeated bouts of Torsades de Pointes (TdP), which were treated with magnesium and ACLS protocols. Both patients ultimately underwent EEG tracings that demonstrated no meaningful brain activity. Care was withdrawn on both patients, who both ultimately died.

Conclusion: Ibogaine administration can result in significant cardiotoxicity manifesting as severely prolonged QTc and TdP, and which can result in cerebral hypoxia and death.

042. Chronic Myoclonus in a Patient Secondary to Ranolazine Miriam Bello, Laura Fil, Faizan Arshad

Healthquest, Poughkeepsie, NY, USA

Background: Ranolazine, sold under the brand name Ranexa ${ }^{\circledR}$ is an active piperazine derivative used to treat chronic angina. It inhibits persistent or late inward sodium current (INa) in the heart, which ultimately reduces tension in the heart wall. A rare side effect associated with ranolazine is myoclonus.

Case Report: This is a single patient chart review. An 84-year-old male with a past medical history including congestive heart failure, chronic angina and renal failure on hemodialysis presents with facial and body twitching, intermittently, for almost a year. This coincides with the start of a new medication, ranolazine. He notices worsening of "twitching" on days he is due for dialysis or late for dialysis. Symptoms would abate after completion of his hemodialysis. His examination in the Emergency Department reveals myoclonic movements of both upper and lower extremities. He has previous work up over the past year for these symptoms including neurology consultations, diagnostic imaging of the brain and spine, as well as functional neurologic studies - all of which have been inconclusive. After review of his medication list by a Medical Toxicologist it was found that the patient was taking ranolazine which could be the cause of his symptoms. After medication discontinuation, his symptoms of twitching rapidly abated and he continues to remain asymptomatic.

Discussion: Ranolazine is primarily excreted renally. We theorize the patient became more symptomatic as the active metabolites accumulated prior to hemodialysis. Earlier recognition of the adverse effects of the medication would have saved the patient from significant symptoms, psychological distress as well as health care cost.

Conclusion: Ranolazine is an antianginal medication that is effective in treating chronic angina. A rare and distressing side effect of ranolazine is myoclonus. This is the second case in the literature of a patient with myoclonus in the setting of ranolazine use.

043. Baclofen Withdrawal after Transition from Intrathecal to Intraventricular Pump

Noah G Berland ${ }^{1}$, Mohamed Jefri ${ }^{1}$, Mehruba Parris ${ }^{1}$, Jeena Jacob ${ }^{1}$, Diane Calello ${ }^{1,2}$

${ }^{1}$ Rutgers NJMS, Newark, NJ, USA. ${ }^{2}$

Background: Baclofen, a GABA-B agonist, is most commonly used to treat chronic spasticity in neuromuscular diseases. Baclofen can be administered directly into the CSF, most commonly via an intrathecal baclofen pump (ITBP). Intraventricular baclofen pump (IVBP) is an alternate option, and generally used after repeated episodes of ITBP failure. However, the optimal dose adjustment after transitioning from an ITBP to an IVBP is not well established.

Methods: This is a single patient chart review of a 13-year-old male with a history of spasticity and an ITBP, previously running at $329.29 \mathrm{mcg} /$ day, who presented for pump failure due to catheter displacement. Vitals: HR 109, BP 136/77, RR 19, and Temp 98.0 (Axillary). He was well appearing and in no apparent distress, although per his mother, he was harder to calm than baseline. Due to catheter displacement the patient had the ITBP replaced with an IVBP.

Results: On POD one, the IVBP was set at $75 \mathrm{mcg} /$ day at neurosurgery recommendation. Medical toxicology was consulted when the PICU became concerned about baclofen withdrawal. On exam the patient was agitated, had myoclonic jerking, and the following vital signs: HR 78162, RR 10-33, BP 57/45-136/110, Temp 98.2-99.8 (axillary). His home medications were continued (clonidine $0.2 \mathrm{mg}$ q6hr, diazepam $5 \mathrm{mg}$ q6hr and carbidopa-levodopa 50-200 q8hr). Baclofen $20 \mathrm{mg}$ via g-tube q6hr for spasticity and a midazolam and dexmedetomidine infusion were also administered for agitation. Non-contrast head CT showed a small intraventricular hemorrhage associated with the catheter and infectious workup (including urine, blood, and CSF) was negative. Neurosurgery titrated his IVBP to $275 \mathrm{mcg} / \mathrm{day}$ on POD nine after which he returned to baseline.

Conclusion: This patient had apparent baclofen withdrawal after transition to intrathecal pump with significant dose reduction. Further research is needed to determine ideal dose adjustments in ITBP. 
044. Drug Reaction with Eosinophilia and Systemic Symptoms (DRESS) Syndrome Secondary to Lenalidomide

Joseph Clemons ${ }^{1}$, Carrisa Ford ${ }^{2}$, Jillian Stariha ${ }^{2}$, Brandon Stein ${ }^{3}$

${ }^{1}$ Oregon Health and Science University, Portland, OR, USA. ${ }^{2}$ Kendall Regional Medical Center, Miami, FL, USA. ${ }^{3}$ Kendall Regional Medical Center, Miami, Florida, USA

Background: Lenalidomide is an antineoplastic drug used to treat relapsed or refractory lymphomas. Previously, five cases of DRESS associated with lenalidomide use have been reported.

Methods: This is a single-patient chart review. A 74-year-old male with a history of chronic lymphocytic leukemia presented with one week of worsening rash accompanied by two days of fever and weakness. Sixteen days prior, he was started on a new oral chemotherapy regimen of rituximab and lenalidomide. No other medication changes were noted. Vitals revealed a febrile male at $38.1^{\circ} \mathrm{C}$, with a heart rate of 103 beats $/ \mathrm{min}$, blood pressure of $82 / 43$, respiratory rate of 16 breaths $/ \mathrm{min}$, and a $\mathrm{SpO} 2$ (99\%). Physical exam revealed diffuse, non-blanching, petechial rash overlying the chest, back, upper/lower extremities, without mucosal involvement. Cervical/axillary lymphadenopathy was noted. Initial labs revealed leukopenia, normocytic anemia, eosinophilia, hyponatremia, hyperkalemia, acute kidney injury, hypomagnesemia, hypoalbuminemia, prolonged PT, and elevated troponin.

Results: Based on history, physical exam, and initial workup, there was a concern for DRESS syndrome secondary to lenalidomide. A biopsy of the left thigh showed interface and spongiotic dermatitis with a superficial and deep perivascular inflammatory infiltrate composed of lymphocytes, histiocytes, neutrophils, and eosinophils. Direct immunofluorescence studies performed on the biopsy of the right thigh failed to demonstrate immunoreactant deposition with IgG, IgA, IgM, C3, C5b-9, and fibrinogen in the epidermis or dermo-epidermal junction, nor around dermal vasculature. These results ruled down conditions such as connective tissue diseases, vasculitis, dermatitis herpetiformis, immunobullous dermatoses, porphyria cutanea tarda, or pseudoporphyria. Dermatopathology results, interpreted within the context of the patient's clinical presentation, were highly suggestive of DRESS syndrome. The patient's symptoms responded well to steroid therapy. He was subsequently discharged home on day six of admission.

Conclusion: DRESS syndrome is a rare but potentially fatal disease that may be associated with lenalidomide.

\section{Seizure after Topical Cyclopentolate}

Suad Khamis Alsulaimani ${ }^{1}$, Waleed ALSukaiti ${ }^{2}$, Hassan AL Bulushi ${ }^{1}$

${ }^{1}$ Emory University, Atlanta, Georgia, USA. ${ }^{2}$ Moh, Ibri, Muscat, Oman Background: Cyclopentolate has been widely used as a mydriatic and rapid onset cycloplegic agent. Systemic toxicities from ophthalmic application have been reported infrequently. A small amount of cyclopentolate eye drops has been reported to induce convulsions in children within an hour of exposure.

Objective: To report a possible association of seizures with the application of cycloplegic ophthalmic drops.

Discussion: A 13-year-old female, with developmental delay but no prior history of seizure, developed a seizure-like activity, within $30 \mathrm{~min}$ of Cyclopentolate $1 \%$ eye drops instillation in both eyes. This occurred during routine ophthalmology evaluation. The seizure was described as up rolling of eyes and jerky movement of upper \& lower extremities that lasted for a few minutes and resolved spontaneously. The patient was transferred to the emergency department of the same hospital in a postictal state. Her vital signs were HR 78/min, BP 106/60 mmhg, RR 18/min, Sat $100 \%$ in RA, Blood Glucose $115 \mathrm{mg} / \mathrm{dl}$. Physical exam was remarkable for somnolence and aphasia. No new focal neurological deficits were noted. Patient observed in the emergency department for eight hours without recurrence of seizure. She had a normal Computed Tomography Scan of the head. Her HGB was $13.8 \mathrm{~g} / \mathrm{dl}$, WBC 7.3 $10 * 3 / \mathrm{ul}$, Platelets $32610 * 3 / \mathrm{ul}$ CRP $0.57 \mathrm{mg} / \mathrm{L}$, Sodium is 137
mmol/Potassium $4.4 \mathrm{mmol} / \mathrm{L}$, Calcium $0.64 \mathrm{mmol} / \mathrm{L}$ creatinine 38.5 umol/L, urea $2.4 \mathrm{mmol} / \mathrm{L}, \mathrm{CK} 108 \mathrm{U} / \mathrm{L}$. Venous Blood Gas showed PH 7.3, Pco2 $45 \mathrm{~mm}(\mathrm{HG}), \mathrm{Hco} 323 \mathrm{mmol} / \mathrm{L}$, lactate $2.5 \mathrm{mmol} / \mathrm{L}$ The patient was discharged after 8 hours of observation for outpatient Pediatric neurology evaluation.

Conclusions: Cyclopentolate eye drops may increase the risk of seizure in the pediatric population. Systemic Toxicities with Cyclopentolate are dose related, doses small as one drop of cyclopentolate $1 \%$ administered thrice to the child at five minutes' intervals causing seizure reported. Caution should be exercised while using topical ophthalmic cycloplegics in children.

046. Use of Intralipid Rescue in Desipramine Overdose: A Case Report

Ryan J Cole ${ }^{1}$, Dayne Laskey ${ }^{2}$, Daniel Patino-Calle ${ }^{3,1}$, Lynn A Farrugia $^{3,1}$, Matthew K Griswold ${ }^{3,1}$

${ }^{1}$ University of Connecticut, Farmington, CT, USA. ${ }^{2}$ University of St. Joseph, West Hartford, CT, USA. ${ }^{3}$ Hartford Hospital, Hartford, CT, USA

Background: Desipramine is a tricyclic antidepressant (TCA) utilized for treatment of depression and off-label for neuropathic pain and attention deficit hyperactivity disorder. It has a lower affinity for $\alpha_{1}$ adrenergic receptors compared to other drugs of this class.

Hypothesis: Intralipid rescue can be employed in desipramine overdose refractory to standard therapy.

Methods: This is a single patient chart review. A 22-year-old male ingested zolpidem and 90 tablets of desipramine $100 \mathrm{mg}$ in a suicide attempt. Upon presentation to the Emergency Department, the patient was unresponsive (GCS four) with BP 136/86 mmHg and an EKG showing sinus tachycardia at $122 \mathrm{bpm}$ with rightward axis deviation, a widened QRS $154 \mathrm{~ms}$, prolonged QTc $524 \mathrm{~ms}$, and a large terminal $\mathrm{R}$ wave in aVR. Physical exam was notable for symmetric pupils four $\mathrm{mm}$ bilaterally without nystagmus, dry skin with axillary sweat, bilateral patellar hyperreflexia without evidence of clonus or extremity rigidity. He was emergently intubated and given $1 \mathrm{mg} / \mathrm{kg}$ activated charcoal through an orogastric tube. Despite 14 ampules of $8.4 \%$ sodium bicarbonate and a sodium bicarbonate infusion, serial EKGs continued to show persistent widening of the QRS up to $200 \mathrm{~ms}$. During therapy, venous $\mathrm{pH}$ rose from 7.43 to 7.55 .

Results: Two boluses of $20 \%$ intralipid emulsion $150 \mathrm{~mL}$ followed by an infusion of $0.25 \mathrm{~mL} / \mathrm{kg}$ over one hour were administered. His course was further complicated by a sustained run of ventricular tachycardia with a pulse during which he was given two boluses of $100 \mathrm{mg}$ lidocaine with good effect. The patient was admitted to the medical intensive care unit and discharged to psychiatric care on hospital day four.

Conclusion: Desipramine overdose can result in severe toxicity that may be refractory to standard treatment methods. Lipid rescue may be especially useful in treating this overdose compared to other tricyclic antidepressants.

\section{Lithium Toxicity: A Case Report of Toxicity Resulting in a} Third-Degree Heart Block

Samantha L Gaetani, Alexandra M Amaducci, Derek Fikse, Andrew L Koons

Lehigh Valley Health Network/USF Morsani College of Medicine, Allentown, PA, USA

Background: Lithium is a medication used to treat bipolar disorder. It has a narrow therapeutic index and its toxicity is frequently seen.

Hypothesis: Hemodialysis and pacemaker placement can combat cardiotoxicity in a patient with lithium toxicity.

Methods: This is a single patient case report. A 66-year-old female with a past medical history of hypothyroidism, diabetes mellitus II, hypertension, and bipolar disorder presented to the emergency department for fatigue, depression, tremors, and decreased appetite and fluid intake. One of her prescribed medications was lithium $300 \mathrm{mg}$ twice daily. An electrocardiogram demonstrated a third-degree heart block. A lithium 
concentration of $>3 \mathrm{mEq} / \mathrm{L}$ [reference range $0.6-1.2 \mathrm{mmol} / \mathrm{L}$, with 3 $\mathrm{mEq} / \mathrm{L}$ the maximum detectable in our laboratory] was detected. A send-out test determined her lithium concentration to be $3.8 \mathrm{mmol} / 1$ [ .5 1.2]. Given that the patient had bradycardia along with prolonged QRS and QTc intervals with lithium toxicity, the medical toxicology service recommended normal saline, two amps of sodium bicarb, and two grams of magnesium sulfate. The patient developed worsening encephalopathy and hemodialysis was recommended.

Results: The patient underwent emergent dialysis, which resulted in improvement of her mental status and laboratory abnormalities. However, the third-degree heart block had not resolved. A permanent pacemaker was placed. Over the previous several days, she had decreased oral intake. This led to dehydration and subsequent renal dysfunction. In addition, this patient was on hydrochlorothiazide, contributing to reduced renal elimination and dehydration and resultant lithium accumulation. This patient was initially somnolent and had tremors consistent with mild symptoms; however, she became encephalopathic and hemodynamically unstable leading to the decision to start hemodialysis.

Conclusion: Lithium is a commonly used medication and can present with a multitude of toxicities, including third-degree heart block resistant to hemodialysis alone.

\section{Human Toxicity from an Injectable Formulation of Cattle and} Swine Ivermectin

Ian Ferguson, Ari B Filip, Michael E Mullins, David B Liss

Washington University, Saint Louis, Missouri, USA

Background: Ivermectin is a human and veterinary antiparasitic agent. In vitro evidence suggests ivermectin may have antiviral activity against SARS-CoV-2. Ivermectin is not currently approved by the FDA for the treatment or prevention of COVID-19.

Research Question: In injectable ivermectin oral ingestion is the associated toxicity secondary to ivermectin or excipients?

Methods: This is a single patient chart review. A 59-year-old male arrived at the emergency department with altered mental status, somnolence, nausea, and vomiting. His wife stated that he was attempting to prevent COVID-19 infection by ingesting veterinary ivermectin. He confused milliliters with ounces and orally ingested $1.5 \mathrm{oz}$ (approximately 45 $\mathrm{mL}$ ) daily for three days. His initial labs were notable for lactate of $>15.0$ $\mathrm{mmol} / \mathrm{L}$, venous $\mathrm{pH}$ of $7.36, \mathrm{CO}_{2} 25 \mathrm{mmol} / \mathrm{L}$, anion gap 14 , serum osmolality $295 \mathrm{mOsm} / \mathrm{kg} \mathrm{H}_{2} \mathrm{O}$, and osmolal gap of nine. He received IV fluids and was admitted to the hospital.

Results: The product ingested was a $10 \mathrm{mg} / \mathrm{mL}$ injectable ivermectin solution for use in cattle or swine in a $250 \mathrm{~mL}$ bottle. The diluent in this formulation is a mixture of approximately $60 \%$ propylene glycol and $40 \%$ glycerol formal. We estimated the patient consumed approximately $450 \mathrm{mg}$ of ivermectin $(4.3 \mathrm{mg} / \mathrm{kg}), 28.1 \mathrm{~g}$ propylene glycol $(269 \mathrm{mg} / \mathrm{kg})$, and $21.7 \mathrm{~g}$ glycerol formal $(207 \mathrm{mg} / \mathrm{kg})$ in each dose. Typical adult dosing of ivermectin for strongyloidiasis is $0.2 \mathrm{mg} / \mathrm{kg}$ once. Maximum daily permissible intake limits are $25 \mathrm{mg} / \mathrm{kg}$ (propylene glycol) and 600 $\mathrm{mcg}$ (glycerol formal). The toxic effects seen in this case likely resulted from the propylene glycol and/or glycerol formal diluents. He returned to baseline within 24 hours with resolution of the hyperlactatemia after supportive care.

Conclusion: We describe a patient with a presentation consistent with propylene glycol toxicity after ingestion of injectable ivermectin in an attempt to prevent COVID-19.

049. Self-Prescribed Horse Ivermectin for COVID-19 Treatment Leading to Fulminant Hepatitis in a Drug-Drug Interaction

Michelle Ji, Bradley Haupts, Anirudh Palicherla

Creighton University, Omaha, NE, USA

Background: Ivermectin is a potent anti-helminthic that people started using for treating COVID-19 after preliminary in-vitro studies demonstrated its potential use as a prophylactic agent against SARSCOV-2. Ivermectin is metabolized in the liver and individuals taking medications, such as Warfarin, may not be aware of the potential for metabolic interactions that have serious consequences.

Hypothesis: Warfarin and ivermectin have a drug-drug interaction that can lead to toxicity.

Methods: This is a single patient case report. A 61-year-old male with a past medical history significant for paroxysmal atrial fibrillation chronically anticoagulated with Warfarin, heart failure, and type two diabetes mellitus, presented to the emergency department with altered mental status and bilateral leg weakness. He admitted that he took two doses of Ivermectin paste intended for horses out of fear he had contracted COVID-19. On admission, the patient was found to have hyponatremia, hyperkalemia, hypochloremia, elevated INR, elevated anion gap acidosis, acute kidney injury, and fulminant hepatitis with transaminitis in the thousands. A transthoracic echocardiogram demonstrated severe heart failure with an ejection fraction at approximately $20 \%$. The patient was treated aggressively with vitamin $\mathrm{K}$ and underwent a right heart catheterization. Later, he was started on a diuretic and milrinone drip with further improvement.

Results: The patient's heart, kidney, and liver function slowly recovered. On the 8th day of treatment, the patient's INR reached 1.8 and his Warfarin was restarted. The patient was encouraged to have an implantable cardioverter defibrillator placed. However, the patient was feeling asymptomatic and wanted to return home, so he left AMA.

Conclusion: If an individual self prescribes medication or supplements without first discussing it with their physician, they may be setting themselves up for severe health consequences because of drug-drug interactions.

050. Altered Mentation Requiring Intubation After Repurposed Ivermectin and Clorsulon Use in an Attempt to Prophylax COVID-19 Lesley Pepin ${ }^{1}$, HoanVu Nguyen ${ }^{1}$, Nicklaus Brandehoff ${ }^{1}$, Jennie C Buchanan $^{1,2}$

${ }^{1}$ Rocky Mountain Poison and Drug Safety, Denver, CO. ${ }^{2}$ Denver Health and Hospital Authority, Denver, CO

Background: Interest in repurposed medications for COVID-19 has gained popularity. One example, ivermectin, can be easily accessed in veterinary formulations. Animal preparations often contain xenobiotics not intended for humans such as clorsulon, an inhibitor of fluke glycolytic enzymes. We hypothesize, large ivermectin ingestions and co-ingestion of other veterinary xenobiotics may lead to clinically significant presentations.

Methods: This is a single patient chart review of ivermectin and clorsulon overdose. A 50-year-old female presented after ingestion of 74 milliliters of a liquid livestock formulation of clorsulon and ivermectin for COVID19 prophylaxis (5.4 mg/kg ivermectin, $54 \mathrm{mg} / \mathrm{kg}$ clorsulon).

Results: Seven hours after ingestion, the patient presented with vomiting and altered mentation. Vital signs were temperature $36.2^{\circ} \mathrm{C}$, heart rate 80 89 , blood pressure $92 / 66-185 / 110 \mathrm{mmHg}$, and $\mathrm{SpO} 287-100 \%$. Initial labs showed blood glucose $165 \mathrm{mg} / \mathrm{dL}$, bicarbonate $25 \mathrm{mmol} / \mathrm{L}$, creatinine $1.2 \mathrm{mg} / \mathrm{dL}$, arterial $\mathrm{pH} 7.31$ with $\mathrm{pCO} 252.8 \mathrm{mmHg}$, negative COVID-19 PCR, negative urine drug screen and negative acetaminophen, salicylate, and ethanol concentrations. The patient's mental status declined necessitating intubation (nine hours post ingestion). Due to crowding, the patient was transferred to another state, where the toxicology service was consulted. After transfer, vitals remained stable. Electroencephalogram showed no seizure. Additional labs obtained lactate $1.9 \mathrm{mmol} / \mathrm{L}$ and creatinine kinase $511 \mathrm{U} / \mathrm{L}$. Recommendations included supportive care and monitoring for acidosis or glucose abnormality. Patient was extubated after 28 hours. The remainder of her hospitalization, she had no further neurologic symptoms or metabolic derangements.

Conclusion: Use of ivermectin in prevention and treatment of COVID19 is on the rise. While reported cases are predominantly benign, severe 
manifestations may result. Exposure to veterinary preparations may pose unique risk given co-exposures to veterinary medications and dosing error due to concentrated formulas designed for livestock administration.

\section{Delta-8 Tetrahydrocannabinol Induced Transient Neurological Deficits}

Zachary Oliff, Joshua Nogar

Northwell Health, Manhasset, NY, USA

Background: The non-uniform process of synthesizing delta-8tetrahydrocannabinol (THC) products results in many impurities and potentially delta- 9 as well as delta-10 isomers. Little is known about the impurities in these products, as well as the long-term effects.

Hypothesis: Commercially available delta- 8 -THC products contain varying amounts of delta- 8 and delta-9-THC than advertised with unpredictable effects.

Methods: This is a single patient chart review. A 59-year-old female with a past medical history of hypertension presented to the Emergency Department after developing paresthesias and weakness of her bilateral upper extremities after ingestion of a product containing " $30 \mathrm{mg}$ delta- 8 THC." The patient and her husband both consumed the same product, but only one patient became symptomatic. Basic labs and a CT head were unremarkable. A quantitative "serum THC level" was sent to the hospital laboratory, and a separate urine sample was sent to an independent laboratory for quantitative assessment of both delta-8-THC and delta-9-THC isomers. The patient was treated symptomatically and ultimately made a full recovery.

Results: Conventional LC-MS/MS analysis of the serum for delta-9-THC resulted in a level of $9 \mathrm{ng} / \mathrm{mL}$, but [per the hospital laboratory] "a reliable result could not be obtained due to the presence of interfering substances." The second laboratory was able to identify an approximate 10 -fold difference in delta-9-THC vs delta-8-THC, at $98 \mathrm{ng} / \mathrm{mL}$ and $1318 \mathrm{ng} / \mathrm{mL}$ respectively, in the urine sample.

Conclusion: Edible cannabis products may contain varying quantities of delta-8 and delta-9-THC, despite product labeling that would suggest otherwise. Furthermore, delta- 8 -THC may be undetectable by the analytical techniques available to most clinicians, and it may exert clinical effects different from that of delta-9-THC.

052. Clinical Effects, Outcomes, and Demographics of Wart and Callus Remover Ingestions Reported to US Poison Control Centers from 2009-2019

Mackenzie Stout ${ }^{1}$, Casey R Tak ${ }^{1}$, Amberly R Johnson ${ }^{2,1}$

${ }^{1}$ University of Utah College of Pharmacy, Salt Lake City, UT, USA.

${ }^{2}$ University of Utah Poison Control Center, Salt Lake City, UT, USA

Background: Unintentional taste/licks of wart and callus removers are often considered benign due to their dilute nature; however, case reports have described major adverse effects following ingestion. This study describes the clinical effects, outcomes, and demographics of wart and callus remover ingestions reported to US Poison Control Centers from 2009-2019.

Methods: A retrospective review of wart and callus remover ingestions was performed. Exposures involving polysubstance, outcomes with confirmed non-exposures, or unrelated effects were excluded. A multiple logistic regression model examined the impact that demographic and clinical characteristics had on moderate or severe outcomes compared to mild or no outcomes. Data were analyzed in SAS v9.4 (SAS Institute, Cary, NC).

Results: A total of 10,390 ingestions were included. The mean age was 9.5 years, and the median age was two years (IQR 3.33) with 51\% female exposures. Salicylic acid was the most common active ingredient ingested $(52.1 \%, \mathrm{n}=5,418)$. Known outcomes were reported in $39 \%(\mathrm{n}=4,150)$ of cases. Moderate effects were seen in $1 \%(n=168)$ of cases and major effects in $0.01 \%(n=16)$. Overall, oral irritation $(n=1,015,9.7 \%)$ was the most common clinical effect reported, but in major and moderate exposures, esophageal injury $(\mathrm{n}=10,62.5 \%)$ and oral burns $(\mathrm{n}=46$, $27.4 \%$ ) were most common, respectively. The multiple logistic regression model found that call site (healthcare facility vs. residential; AOR: 11.3, 95\% CI 8.2-15.7), reason (adverse reaction vs. unintentional general; AOR: 7.1, 95\% CI 2.3-21.4), and product type (unknown vs. salicylic acid; AOR: $2.9,95 \%$ CI 2.1-4.0) were the most significant predictors of moderate or severe outcomes.

Conclusion: Major and moderate outcomes were rare following ingestion of wart and callus removers; however, specific product types, reason, and call site were the most significant predictors of these outcomes.

053. Rivastigmine to the Rescue - Diphenhydramine Intoxication Treated with Rivastigmine During the National Physostigmine Shortage

Demi C Galindo, Jeff H Kamta, Rachel Gorodetsky, Elizabeth A Kadow, Timothy J Wiegand

University of Rochester Medical Center, Rochester, NY, USA

Background: Physostigmine is a centrally acting carbamate, and effective antidotal therapy for anticholinergic toxicity. A national supply shortage of physostigmine began in early 2019. Rivastigmine, the only other commercially available centrally acting carbamate, represents an alternative therapeutic.

Hypothesis: Rivastigmine represents an alternative antidote to physostigmine for select overdoses involving anticholinergic agents during the current physostigmine shortage.

Methods: Single patient chart review. A 25-year-old female presented after ingesting approximately 30 tablets of $25 \mathrm{mg}$ diphenhydramine. She was disoriented, hallucinating, and not responding to questions. She exhibited 'picking behavior,' had decreased bowel sounds, dry mucous membranes, anhidrosis, urinary retention, clonus, and tachycardia. She had a witnessed seizure, $<60$ seconds, and was given two mg lorazepam intravenously. Labs revealed a metabolic acidosis with anion gap, which resolved with lactated ringer's solution $\left(\mathrm{CO}_{2} 15 \mathrm{mmol} / \mathrm{L}\right.$, anion gap 22). Electrocardiogram showed QTc and QRS intervals $483 \mathrm{~ms}$ and $87 \mathrm{~ms}$, respectively. She was admitted to the ICU; telemetry demonstrated ventricular ectopy with pre-ventricular contractions and occasional bigeminy. The patient's delirium and hallucinations persisted.

Results: Three milligram rivastigmine was administered via a nasogastric tube. The patient's mental status improved within two hours of administration, and she was able to recall the events leading up to the ingestion. Within three hours she was fully alert and oriented. There were no adverse effects with rivastigmine administration. Her improved mental status was sustained through discharge on hospital day three, after psychiatry consultation.

Conclusion: Rivastigmine has a slower onset of action compared to physostigmine, with peak effect at 1 hour versus 10-12 minutes; and longer duration of action of 10 hours vs. $60-90$ minutes. A three mg single dose produced sustained reversal of anticholinergic delirium/hallucinations. This is congruent with the few other existing case reports regarding rivastigmine use in this manner. Further research is warranted.

054. Improvement of Visuospatial Processing After Rivastigmine Administration in Antimuscarinic Poisoning Matthew S Correia ${ }^{1,2}$, Robert G Hendrickson ${ }^{1,2}$

${ }^{1}$ Oregon Health and Science University, Portland, OR, USA. ${ }^{2}$ Oregon Poison Center, Portland, OR, USA

Background: Overdose of first-generation antihistamine medications frequently precipitate antimuscarinic toxicity. Central nervous effects are epitomized by floccillation, delirium, and agitation. However, symptoms of confusion can be more subtle encompassing impairment of executive function, lack of attention, and diminished visuospatial processing. Physostigmine is the antidote of choice because it treats both the peripheral and central antimuscarinic effects and has previously been demonstrated to reverse the visuospatial impairment in toxicity. 
Case: A 20-year-old female presented to the emergency department after an intentional ingestion of an unknown amount of diphenhydramine and ethanol. On arrival, her pulse was 117 beats per minute, respiratory rate 20 breaths per minute, blood pressure $136 / 92 \mathrm{mmHg}$, oxygen saturation $100 \%$ on room air, and she was normothermic. Electrocardiogram was notable for a QRS of $83 \mathrm{~ms}$ and QTc of $469 \mathrm{~ms}$. She required four milligrams of intravenous lorazepam for agitation over the ensuing six hours but continued to exhibit central and peripheral antimuscarinic symptoms including floccillation, garbled speech, and inattention. Upon evaluation by toxicology, she was prompted to draw a clock which was markedly abnormal. Due to a hospital shortage of physostigmine, six mg of rivastigmine was recommended. Upon re-evaluation 1.5 hours after medication administration ( 3.5 hours after initial evaluation) her sensorium, peripheral symptoms, and ability to accurately draw a clock were dramatically improved. She required no further medication and was discharged later that evening.

Discussion: Rivastigmine is a newer centrally acting acetylcholinesterase inhibitor, initially approved for dementia. Off-label application for antimuscarinic poisoning and delirium has grown in the setting of recent national physostigmine shortages. We noted marked improvement of delirium, concentration, and visuospatial processing as demonstrated by the patient's ability to accurately draw a clock after administration of rivastigmine. Conclusion: Rivastigmine may be considered as an alternative off-label agent to reverse symptoms of anticholinergic delirium, particularly when physostigmine is not available.

055. Adverse Outcomes in Topical Lidocaine Exposure: A Pediatric Case Series from the United States (US) National Poison Data System (NPDS)

Jonathan R Eisenberg ${ }^{1}$, Natalie J Tedford ${ }^{1}$, Sarah M Becker ${ }^{1}$, Nicholas Weaver $^{1}$, Michael J Moss ${ }^{1,2}$

${ }^{1}$ University of Utah, Salt Lake City, UT, USA. ${ }^{2}$ Utah Poison Control, Salt Lake City, UT, USA

Background: Lidocaine toxicity exists on a spectrum from sensory changes to alterations in mental status to seizures, tachydysrhythmias, and cardiac arrest. Toxic dose is approximately $4.5 \mathrm{mg} / \mathrm{kg}$ but may be altered by site of application, regional blood flow, rate of absorption, or adjuncts. Early symptoms include dizziness, tinnitus, circumoral numbness, and blurry vision. If effects continue, seizures, cardiac arrest, and death can result. Although injected lidocaine has a higher rate of complication, topical preparations can also induce serious toxic effects.

Methods: This is a retrospective case series of patients aged 0-18 years with topical lidocaine toxicity from 2006-2020. The National Poison Data System (NPDS) was queried for moderate/major outcomes following topical lidocaine exposure. Patients discharged home or without secondary effect from the exposure were excluded. Individual case records were then requested from poison centers.

Results: Of 37 cases identified, 25 had moderate effects (67.6\%), 10 had major effects $(27 \%)$, and two deaths $(5.4 \%)$. Mean age was five years 16 days. The most common symptoms included cyanosis (29.7\%), seizures (18.9\%), CNS depression (13.5\%), drowsiness/lethargy (13.5\%), and tachycardia $(10.8 \%)$. The most common therapies were dilution/ irrigation (35.1\%), IV fluids (29.7\%), oxygen (29.7\%), methylene blue $(27 \%)$, benzodiazepines $(13.5 \%)$, and intubation (10.8\%). ICU disposition was $35.1 \%$. Case details were requested for 37 cases, 16 cases (43.2\%) were provided. Of the two deaths, one had significant cardiac history. The most common use of topical lidocaine was at home prior to a dermatologic procedure (37.5\%).

Conclusions: Although rare, topical lidocaine can induce serious outcomes resulting in ICU level care or death. Given frequent use, especially in outpatient settings, greater vigilance should be taken with prescriptions, instructions for use, and anticipatory guidance. Outcomes amongst those with moderate/major effects were generally positive without comorbidities and the vast majority of patients were safely discharged home.
056. Pericarditis Associated With Acute Acetaminophen Overdose Michael Kosofsky, Lauren T Murphy

Temple University Hospital, Philadelphia, PA, USA

Background: Acetaminophen is one of the most commonly encountered medications in overdose. Though hepatic injury from toxicity is well recognized, myocardial infarction, heart failure, myocarditis, and pericarditis are observed in rare reports. We present a patient who developed pericarditis in the acute setting of acetaminophen overdose.

Case Report: A 28-year-old presented after intentional acetaminophen ingestion. The exact time of ingestion was unknown. On arrival he reported abdominal pain and episodes of vomiting. Initial vital signs were unremarkable. Pertinent initial labs included an acetaminophen concentration of $43 \mathrm{ug} / \mathrm{ml}$, AST $53 \mathrm{U} / \mathrm{L}$, ALT of $74 \mathrm{U} / \mathrm{L}$, INR of 1.7, and an undetectable troponin. $\mathrm{N}$-acetylcysteine infusion was started. Ten minutes later the patient developed severe chest pain and an initial electrocardiogram (ECG) displayed regional ST segment elevation and PR depression, which progressed diffuse changes on repeat ECG 40 minutes later. Cardiology performed an echocardiogram displaying normal systolic function without wall motion abnormalities, no pericardial effusion, and diagnosed the patient with pericarditis. Cardiology deferred empiric treatment as inflammatory markers were normal and his symptoms had improved. On hospital day three, ALT and AST peaked at 3,459 U/L and 1,287 U/L, respectively. The patient completed $\mathrm{N}$-acetylcysteine therapy on day six before making a complete recovery. ECG at discharge showed improved ST segment elevation and PR depression.

Discussion: There is currently no causative link between acetaminophen and cardiotoxicity. Previous cases of cardiac injury associated with acetaminophen toxicity occurred simultaneously with severe hepatotoxicity and encephalopathy. Proposed mechanisms include a hypersensitivity reaction leading to drug-induced pericarditis, or NAPQI formation and free radical generation in the pericardium and myocardium that causes cellular damage.

Conclusion: Pericarditis may be observed with acetaminophen toxicity. In addition to thorough laboratory testing, an ECG should be considered in the evaluation of acetaminophen overdose to monitor for potential cardiac injury.

057. Accidental Intravenous Acetaminophen Overdose in an Infant Without Development of Hepatotoxicity

Nathan Friedman ${ }^{1,2}$, Daniel Lasoff ${ }^{1}$

${ }^{1}$ University of California - San Diego, San Diego, CA, USA. ${ }^{2}$ VA San Diego Healthcare System, San Diego, CA, USA

Background: Therapeutic errors involving acetaminophen may result in a substantial overdose in infants owing to their relatively low body weight. The toxic dose of intravenous acetaminophen is not wellestablished in this population, with hepatotoxicity reported at a level of $90 \mathrm{mg} / \mathrm{kg}$ in one study. Some authors suggest empiric treatment with Nacetyl cysteine with doses as low as $60 \mathrm{mg} / \mathrm{kg}$.

Methods: This is a case report. A 13-month-old male weighing 8.8 kilograms, born at 35 weeks but otherwise healthy was admitted to our pediatric hospital ICU for viral bronchiolitis requiring high-flow oxygen therapy. Due to a dosing error, he was inadvertently given $1000 \mathrm{mg}$ (110 $\mathrm{mg} / \mathrm{kg}$ ) of acetaminophen intravenously over approximately a 90-minute period. The error was recognized seven hours later.

Results: Serial acetaminophen levels were drawn along with hepatic function panels. A seven-hour acetaminophen level was $40 \mathrm{ug} / \mathrm{mL}$ and an eleven-hour level was below the limit of detection. Liver function tests were normal at both time points. No antidotal therapy was started. The patient was discharged that day with improved respiratory status and no other sequelae.

Conclusion: Additional research is needed to correlate dosage of intravenous acetaminophen with serum levels and development of 
hepatotoxicity. In this case, a dose of $110 \mathrm{mg} / \mathrm{kg}$ did not result in hepatotoxicity.

058. Delayed Acetaminophen Poisoning Treated with Adjunctive 4Methylpyrazole Therapy

Marielle A Brenner ${ }^{1,2}$, Robert S Avera ${ }^{1,2}$

${ }^{1}$ Indiana University, Indianapolis, IN, USA. ${ }^{2}$ Indiana Poison Center, Indianapolis, IN, USA

Background: 4-methylpyrazole (fomepizole) is emerging as an adjunctive therapy for acetaminophen poisoning due to its inhibition of CYP2E1, the enzyme responsible for conversion of acetaminophen to its toxic metabolite, N-acetyl-p- benzoquinone imine (NAPQI). Most cases have been reported in acute ingestions with little literature on efficacy in delayed presentations. Study Aim: Demonstrate the use of 4methylpyrazole in delayed acetaminophen poisoning.

Methods: This is a single patient chart review. A 67-year-old female presented after being found down for an unknown amount of time, but estimated at less than 12 hours, with empty bottles of over-the-counter acetaminophen $(325 \mathrm{mg})$ containing cold medicine and extra strength acetaminophen $(500 \mathrm{mg})$. Notable initial vital signs included temperature $35.5^{\circ} \mathrm{C}$, heart rate 101 beats per minute, and blood pressure $119 / 57 \mathrm{mmHg}$. On the initial exam, she was unresponsive and intubated for airway protection. Significant initial laboratory findings included acetaminophen $292 \mathrm{mcg} / \mathrm{mL}$, INR $1.76 \mathrm{IU}$, AST $132 \mathrm{U} / \mathrm{L}$, ALT $139 \mathrm{U} / \mathrm{L}$, creatinine $2.53 \mathrm{mg} / \mathrm{mL}, \mathrm{pH} 6.83, \mathrm{pCO} 244 \mathrm{mmHg}, \mathrm{HCO} 35.4 \mathrm{mmol} / \mathrm{L}$, and creatinine kinase $52 \mathrm{U} / \mathrm{L}$. She was started on norepinephrine for hypotension after intubation.

Results: 4-methylpyrazole was started five hours after presentation at the guidance of a medical toxicologist. Other therapies included Nacetylcysteine at a continuous rate of $12.5 \mathrm{mg} / \mathrm{kg} / \mathrm{hr}$ and continuous venovenous hemodiafiltration. 4-methylpyrazole was dosed at standard dosing of $15 \mathrm{mg} / \mathrm{kg}$ followed by $10 \mathrm{mg} / \mathrm{kg}$ every 12 hours. Her ALT and AST concentration peaked at $270 \mathrm{U} / \mathrm{L}$ and $246 \mathrm{U} / \mathrm{L}$, respectively. She was discharged with complete resolution of her liver and renal injury. Conclusion: This was delayed presentation of intentional acetaminophen poisoning with severe metabolic acidosis, renal injury, and early liver injury who was treated with adjunctive 4-methylpyrazole, leading to apparent prevention of more significant liver injury.

\section{Fomepizole as Treatment Adjunct After Massive Acetaminophen} Overdose With Delay to Treatment

Jack A Finnegan, Yiraima Medina, Matthew Slane

Kendall Regional Medical Center, Miami, FL, USA

Background: Despite widespread usage of an existing robust antidote, massive acetaminophen ingestion is associated with significant morbidity and mortality. Prior studies have suggested a probable role for fomepizole as adjunct therapy.

Case Description: A 16-year-old female presented to our pediatric emergency department $25 \mathrm{hrs}$ after one time ingestion of over 50 grams (649 $\mathrm{mg} / \mathrm{kg}$ ) of acetaminophen tablets. Initial acetaminophen serum level was obtained 25 hours after ingestion showing a concentration of $188.6 \mathrm{mcg} /$ $\mathrm{mL}$. Local Poison Control was contacted, and the patient was initiated on oral n-acetyl cysteine therapy as well as a single dose of intravenous fomepizole $15 \mathrm{mg} / \mathrm{kg}$ before being admitted to our PICU. AST and ALT concentrations peaked four days after ingestion at 6847 and 5993 units/L respectively. INR also peaked at day four at 2.8 before normalizing. The patient did not progress to renal failure, clinically significant coagulopathy nor encephalopathy. The patient was discharged in good health on post-ingestion day ten.

Conclusion: Massive acetaminophen overdose and delay to antidotal therapy are both independent predictors of poor prognosis in acetaminophen toxicity. It is not uncommon for patients with similar presentations to progress to multi-organ failure requiring both hemodialysis and liver transplant for an attempt at survival despite appropriate administration of n-acetyl cysteine. Fomepizole has been shown to prevent formation of reactive metabolites through inhibition of cytochrome P450 enzymes and to attenuate liver cell apoptosis mediated by c-Jun N-terminal kinase. Anti-apoptotic effects may be of critical utility in cases of delayed nacetyl cysteine administration in which reactive metabolites have already formed. This patient's outstanding clinical course suggests a role for adjunct fomepizole therapy in select patients with significant delay to antidotal therapy.

060. Diphenhydramine-Induced Antimuscarinic Delirium Treated with Physostigmine and Transdermal Rivastigmine James D Whitledge ${ }^{1,2}$, Christopher J Watson ${ }^{1,2}$, Michael D Simpson ${ }^{1,2}$, Azza B Bakkar ${ }^{3}$, Leora S Boussi ${ }^{3}$, Michael J Scott ${ }^{3}$, Katherine L Boyle ${ }^{3}$ ${ }^{1}$ Boston Children's Hospital, Boston, MA, USA. ${ }^{2}$ Harvard Medical Toxicology Fellowship, Boston, MA, USA. ${ }^{3}$ Beth Israel Deaconess Medical Center, Boston, MA, USA

Background: Antimuscarinic delirium is typically treated with physostigmine, which is on national shortage. Transdermal rivastigmine, a tertiary amine cholinesterase inhibitor commonly prescribed to dementia patients, may represent a long-acting alternative to physostigmine.

Hypothesis: Transdermal rivastigmine may be an effective adjunct or alternative to physostigmine for the treatment of antimuscarinic delirium. Methods: This is a case report. A 48-year-old female presented to an urban academic hospital emergency department after ingesting approximately 150 pills of $25 \mathrm{mg}$ diphenhydramine in a suicide attempt. A medical toxicologist evaluated the patient at bedside and found her to have antimuscarinic delirium. To avoid intubation, two milligrams of physostigmine were administered intravenously with significant response. The patient required an additional one milligram IV of physostigmine prior to ICU transfer for recurrent symptoms. On day two, she again developed recurrent delirium and was unable to tolerate oral medications. No further physostigmine was available because of drug shortages. A $9.5 \mathrm{mg}$ transdermal rivastigmine patch was applied 22 hours post-presentation. Serum diphenhydramine concentrations were obtained.

Results: The serum diphenhydramine concentration on patient presentation to the ED was $1500 \mathrm{ng} / \mathrm{mL}$ (therapeutic range $25-112 \mathrm{ng} / \mathrm{mL}$ ). At hour 25 , the level remained elevated at $760 \mathrm{ng} / \mathrm{mL}$, consistent with sustained toxicity. Two hours after rivastigmine administration, the patient's mental status markedly improved. The patch was removed after 24 hours, at which time she was asymptomatic. She never developed cholinergic symptoms.

Conclusion: This case illustrates successful treatment of diphenhydramine-induced antimuscarinic delirium with intravenous physostigmine and transdermal rivastigmine. While the use of oral rivastigmine to reverse antimuscarinic delirium has been described, this report highlights the use of physostigmine and transdermal rivastigmine in combination. Transdermal rivastigmine may be an effective and longlasting adjunct or alternative for antimuscarinic delirium when physostigmine supply is limited.

061. Analysis of Methanol in Blood, Vitreous Humor and Cerebrospinal Fluid using GC-HS - A Case Study

Neha Afaria ${ }^{1}$, Sudhir Kumar Gupta ${ }^{1}$, Ashok Kumar Jaiswal ${ }^{1}$, Rohit Kanojia $^{2}$

${ }^{I}$ All India Institute of Medical Sciences, Delhi, India. ${ }^{2}$ University of Delhi, Delhi, India

Background: Methanol can be encountered in clinical and forensic cases and its toxicity is primarily related to its metabolic products- formaldehyde and formic acid, that can cause permanent neurologic dysfunctions, severe metabolic disturbances, ocular morbidity up to blindness, or even death. Quantitative determination and interpretation of methanol levels is imperative in clinical and forensic toxicology. Hypothesis: Gas chromatography- Headspace (GC-HS) can be used to determine methanol concentration in different biological matrices. Methods: This is a case 
study. An approx. 35-year-old male with a history of becoming unconscious following two episodes of vomiting at his residence. He was taken to hospital where he was declared dead. The deceased has a history of chronic alcohol intake, and he sustained several self falls due to alcoholism. Blood, vitreous humor and cerebrospinal fluid (CSF) samples were taken for toxicological examination. The samples were run in GC-HS for analysis of methanol. The analysis only required one $\mathrm{ml}$ of sample and addition of internal standard (IS). Total runtime of analysis (one analyte, one IS) was achieved within 20 min.

Results: Methanol was successfully detected in blood, vitreous humor and CSF of the deceased, which was more than $100 \mathrm{mg} / \mathrm{ml}$ using GC-HS. Conclusion: GC-HS is efficient for the measurement of methanol concentrations, owing to its high reliability, specificity, sensitivity, and reproducibility. Multiple specimens including those that are more resistant to post-mortem changes should be collected and tested in cases where a blood sample is not readily obtainable due to alterations, putrefaction, etc. GC-HS can be used to analyze methanol levels in blood, vitreous humor and CSF.

062. Tranexamic Acid (TXA)-Associated Multifocal Myoclonic Seizures

Kai-Wen Cheng

Surgical Intensive Care Unit, DaLin TzuChi Hospital, Buddist TzuChi Medical Foundation, ChiaYi County, Taiwan. Department of Clinical Toxicology and Occupational Medicine, Taipei Veterans General Hospital, Taipei City, Taiwan

Background: Generalized seizures are well-known complications of intravenous TXA for hemostasis. It is less associated with multifocal myoclonic seizures.

Hypothesis: TXA-associated seizures could present as multifocal myoclonic movements.

Methods: This is a single-patient chart review. The 80 -year-old woman, with a history of chronic kidney disease, stage IV, and without a history of epilepsy or recent head injury, suffered from acute lower gastrointestinal bleeding. In the emergency department, her serum level of creatinine was $3.05 \mathrm{mg} / \mathrm{dL}(269.62 \mu \mathrm{mol} / \mathrm{L})$, and BUN was $26.4 \mathrm{mg} / \mathrm{dL}(9.42 \mathrm{mmol} / \mathrm{L})$. She received intravenous TXA (1000 mg every eight hours) for hemostasis and was admitted to the general ward. This madam experienced cluster focal seizures (myoclonic jerks at left upper and lower extremities) about an hour after the sixth dose of TXA and was transferred to the intensive care unit (ICU). Serum and urine TXA levels were obtained (analyzed through liquid chromatography-tandem mass spectrometry [LC-MS/MS], the limit of detection: $50 \mathrm{ng} / \mathrm{mL}$ ) to confirm the association of focal seizures and supratherapeutic drug levels.

Results: Five hours after the first focal seizures, an episode of self-limited generalized tonic-clonic seizures (GTCS) was observed in the intensive care unit, and the serum TXA level was $84 \mu \mathrm{g} / \mathrm{mL}$ (urine level $45.2 \mu \mathrm{g}$ / $\mathrm{mL}$ ). It decreased to $10.6 \mu \mathrm{g} / \mathrm{mL} 33$ hours after the first focal seizures and was not detectable in serum after 57 hours. An episode of focal myoclonic seizures involving the right upper extremity, opposite to the side of cluster focal seizures, was noted several hours after the GTCS. There were no definite epileptic discharges noted in the electroencephalogram four days later.

Conclusion: TXA-associated seizures could present as multifocal myoclonic movements.

063. Normalization of R-Time Following Rescue Therapy with Andexanet Alfa in the Setting of Apixaban Overdose and Traumatic Intracranial Hemorrhage

Abigail F Kerns, John DelBianco, Daniel Jenniches, Matthew P Stripp, Allan S Philp

Allegheny Health Network, Pittsburgh, PA, USA

Background: Apixaban is a direct factor Xa inhibitor that can result in life-threatening bleeding. Reversal strategies include prothrombin complex concentrate (PCC) and andexanet alfa. Anti-inhibitor coagulant complex (FEIBA) is an activated PCC which overcomes factor X inhibition by supplementing clotting factors. Andexanet alfa is a factor $\mathrm{Xa}$ decoy that directly binds factor Xa inhibitors. Optimal management of traumatic intracranial hemorrhage (ICH) in the setting of apixaban overdose is unknown.

Methods: This case report describes a 69-year-old female who presented due to a ground level fall resulting in traumatic ICH. Patient reportedly ingested apixaban $275 \mathrm{mg}$, carvedilol $250 \mathrm{mg}$, atorvastatin $1200 \mathrm{mg}$, ethanol, and unknown amounts of amlodipine. FEIBA was administered. Imaging at six hours showed new areas of ICH. Additional FEIBA was given (cumulative dose: 50 units $/ \mathrm{kg}$ ). Imaging eleven hours after the initial scan showed ICH expansion and worsening mass effect. Postreversal thromboelastography (TEG) studies demonstrated ongoing coagulopathy. Repeat FEIBA doses were given (cumulative dose: 100 units $/ \mathrm{kg}$ ). The patient underwent decompressive craniotomy. Postoperative imaging showed expansion of existing ICH and new areas of hemorrhage. Andexanet alfa became available and was given at the high-dosing regimen. No further expansion of ICH was observed and Rtime on TEG normalized.

Results: This patient demonstrated ongoing coagulopathy and worsening traumatic ICH in the setting of apixaban overdose despite repeated FEIBA administration. After rescue therapy with high dose andexanet alfa, coagulation parameters improved, and ICH expansion halted. The patient was ultimately discharged to rehabilitation with a Glasgow Coma Scale score of fifteen. She resumed apixaban for nonvalvular atrial fibrillation three months later.

Conclusion: In this case of traumatic ICH complicated by apixaban overdose, despite repeated FEIBA administration, the patient continued to have laboratory and radiographic evidence of ongoing hemorrhage. Coagulation parameters improved and no evidence of worsening ICH was observed after andexanet alfa administration.

064. Metabolic Acidosis with an Elevated Lactate Concentration Managed with Continuous Venovenous Hemodiafiltration with Serial Multicompartmental Metformin Analyses: Confirmation of Removal Daniel J McCabe ${ }^{1,2}$, Nicholas R Lind ${ }^{1}$, Joshua B Radke ${ }^{1,2}$, Bryan Z Wilson ${ }^{1,2}$

${ }^{I}$ University of Iowa, Iowa City, IA, USA. ${ }^{2}$ Iowa Poison Control Center, Sioux City, IA, USA

Background: Metabolic acidosis with an elevated lactate concentration (MALA) is a rare but life-threatening condition which can occur after metformin overdose. Despite a large volume of distribution metformin is dialyzable but hemodynamic instability may impede the use of intermittent hemodialysis, forcing reliance on continuous renal replacement therapy with minimal available data on metformin removal via this therapy.

Case: A 41-year-old male ingested two bottles of metformin and arrived at the hospital one hour later with slurred speech, tachycardia, nausea. Initial labs were absent metabolic derangements or renal dysfunction. Over the next four hours they became obtunded, hypotensive, hypothermic. Repeat labs five hours post-ingestion were significant for $\mathrm{K}^{+} 5.3 \mathrm{mEq} / \mathrm{L}, \mathrm{HCO}_{3} 13$ $\mathrm{mEq} / \mathrm{L}, \mathrm{Cr} 1.50 \mu \mathrm{mol} / \mathrm{L}$, lactate $11.2 \mathrm{mmol} / \mathrm{L}$, and arterial $\mathrm{pH} 7.08$. At this time, they required large-volume fluid resuscitation, vasopressor support, and intubation. Twelve hours post-ingestion (on isotonic sodium bicarbonate infusion) the following abnormalities were present: $\mathrm{K}^{+} 6.0 \mathrm{mEq} / \mathrm{L}$ $\mathrm{HCO}_{3} 10 \mathrm{mEq} / \mathrm{L} \mathrm{Cr} 2.0 \mu \mathrm{mol} / \mathrm{L}$ Glu $18 \mathrm{mg} / \mathrm{dL}$, lactate $21.0 \mathrm{mmol} / \mathrm{L}$, arterial $\mathrm{pH}$ 7.14. Continuous venovenous hemodiafiltration (CVVHDF) was initiated at this time (blood flow rate $350 \mathrm{ml} / \mathrm{min}$ ). Comatose state, hemodynamic instability, oliguria, and metabolic derangements all corrected within thirty hours post-initiation of CVVHDF.

Results: High performance liquid chromatography/tandem mass spectrometry was completed at time of initiation, and 12-hours post-initiation, of CVVHDF for metformin concentration from multiple sites. PreCVVHDF (blood: $63 \mathrm{mcg} / \mathrm{ml}$ ) (urine: $>50 \mathrm{mcg} / \mathrm{ml}$ ). Twelve hours 
post-CVVHDF-initiation (blood: $43 \mathrm{mcg} / \mathrm{ml}$ ) (dialysate: $47 \mathrm{mcg} / \mathrm{ml}$ ) (urine: $>50 \mathrm{mcg} / \mathrm{ml}$ ).

Conclusion: There has been uncertainty whether CVVHDF improves MALA by merely improving acid-base physiology allowing continued elimination or if CVVHDF removes significant metformin from the circulation. In addition to the undeniable improvement of acid-base status which allows continued renal elimination, this case supports some metformin removal via CVVHDF.

065. Temporal Changes in Therapies Used for Intentional BetaAdrenergic Antagonist Ingestions Reported to the National Poison Data System

Christopher J Watson ${ }^{1,2}$, Michael C Monuteaux ${ }^{1}$, Michele M Burns ${ }^{1}$

${ }^{1}$ Boston Children's Hospital, Boston, MA, USA. ${ }^{2}$ Beth Israel Deaconess Medical Center, Boston, MA, USA

Background: Myriad therapies are used to manage beta-adrenergic antagonist (BAA) toxicity, and there is equipoise regarding which are most beneficial. Management strategies are evolving; therapies such as highdose insulin (HDI), lipid emulsion therapy (LET), and extracorporeal membrane oxygenation (ECMO) have emerged over the previous 20 years. We seek to describe temporal changes in therapies used in the management of intentional BAA ingestions.

Methods: Retrospective review of patients with acute intentional singleagent BAA ingestions presenting to a US healthcare facility between 20002020 for whom a Poison Control Center was consulted. Data were abstracted from the National Poison Data System. Using logarithmic regression controlling for age, odds of using therapies for BAA toxicity were assessed over time. Frequencies of usage for 2000-2001 and 2019-2020 were compared. Results: 20,819 cases were identified. Gastrointestinal decontamination, including activated charcoal $(\mathrm{aOR}=0.91,95 \%$ confidence interval 0.90 $0.91)$ and whole bowel irrigation $(0.91,0.88-0.92)$ showed annual decreased odds of use per year. Benzodiazepines (1.06, 1.04-1.08), calcium $(1.13,1.11-1.15)$, dextrose $(1.07,1.05-1.09)$, extracorporeal removal (1.10, 1.04-1.17), glucagon (1.04, 1.03-1.05), HDI (1.12, 1.09-1.14), intravenous fluids $(1.05,1.05-1.06)$, intubation $(1.04,1.03-1.06)$, LET (4.1, 2.0-8.4), magnesium $(4.2,2.9-6.2)$, potassium $(4.0,3.0-5.4)$, and vasopressors $(1.03,1.02-1.05)$ demonstrated annual increased odds of use. The following therapies were used in more than $2 \%$ of cases in either 2000-2001 (2,656) or 2019-2020 (4,825): calcium (2000-2001: $0.2 \%$; 2019-2020: $3.2 \%$ ), charcoal $(50.0 \% ; 15.8 \%)$, dextrose $(0.4 \% ; 2.4 \%)$, lavage $(6.6 \% ; 0.3 \%)$, glucagon $(3.5 \% ; 9.4 \%)$, HDI $(0.15 \% ; 2.5 \%)$, intravenous fluids $(13.6 \% ; 29.5 \%)$, intubation $(0.9 \% ; 2.7 \%)$, and vasopressors $(2.0 \% ; 5.0 \%)$. In 2019-20, LET was used 16 times and ECMO once.

Conclusion: The use of decontamination for intentional BAA ingestions is declining. Most cases do not require cardiovascular support, although the frequency of interventions is modestly increasing. Overall, intentional BAA ingestions in this multiyear national population do not commonly require significant intervention.

066. Supratherapeutic Digoxin Levels after Intracervical Digoxin administration for Surgical Abortion

Muhammad Akbar Baig ${ }^{1}$, Wells Brambl ${ }^{2}$, Joshua Nogar ${ }^{1}$

${ }^{1}$ North Shore University Hospital, Manhasset, NY, USA. ${ }^{2}$ Long Island Jewish Medical Center, New Hyde Park, NY, USA

Background: Digoxin is injected intra-amniotically to produce asystole during surgical abortion. It can be injected transabdominally into the amniotic fluid or transvaginally into the fetus. We report a case of an iatrogenic administration of two $\mathrm{mg}$ digoxin into the maternal cervix during an elective abortion which resulted in supratherapeutic digoxin levels.

Method: A 25-year-old, 23 weeks pregnant patient with no past medical history was referred to the Emergency Department from a clinic where two mg of digoxin was inadvertently administered into the maternal cervix. It was reported the unlabeled digoxin syringe was mistaken for lidocaine. The patient presented asymptomatically with a heart rate of $74 \mathrm{bpm}$ and a blood pressure of 107/57 $\mathrm{mmHg}$. Her electrocardiogram showed a normal sinus rhythm. In the ED, a serum digoxin assay of 12.1 $\mathrm{ng} / \mathrm{ml}$ was measured at two hours post-injection. Six hours post exposure, the digoxin concentration decreased to $2.8 \mathrm{ng} / \mathrm{ml}$. It further decreased to $1.6 \mathrm{ng} / \mathrm{ml}$. at 12 hours. The patient remained aymptomatic throughout her period of observation with a resting heart rate ranging between $70-80 \mathrm{bpm}$ and was normotensive. Digoxin immune FAB werenot indicated for this patient and she was discharged uneventfully.

Result: Digoxin is used as a potent feticidal agent, however minimal toxicokinetic information is available if there were to be aniatrogenic injection into the maternal cervix. In therapeutic dosing, we see a distinct two-compartment distribution model lasting over six hours. Our case report of iatrogenic digoxin injection into the maternal cervix, resulted in elevated pre-distribution digoxin levels followed by a gradual decline over six and twelve hours. The patient remained asymptomatic,therefore she did not require treatment.

Conclusion: Intracervical digoxin is a complication arising from late term abortions. Syringes should always be labeled. Patients should be monitored for digoxin toxicity, but may not necessarily develop toxicity, despite supratherapeutic levels.

067. Cardiac Arrest Following Benzonatate Overdose with a Rapidly Narrowing QRS Interval

Robert J Stephens, Ari B Filip, Kevin T Baumgartner, Evan S Schwarz, David B Liss

Washington University, Saint Louis, Missouri, USA

Background: Benzonatate is a local anesthetic-like sodium channel antagonist that is widely prescribed for cough. The toxic effects of overdose include neurologic and cardiac manifestations. Sodium channel antagonists often result in QRS prolongation on ECG.

Research Question: Do patients following an acute overdose of benzonatate present with narrow or wide QRS complexes on ECG?

Methods: This is a single patient chart review of a 14-year-old girl found in cardiac arrest after benzonatate ingestion. ECG tracings from EMS and inpatient settings, laboratory data, and other clinical data from the patient's admission were reviewed. A brief literature review of ECG abnormalities in benzonatate poisoning was conducted.

Results: The patient was last seen normal four minutes before being found in cardiac arrest by her father. She received CPR from her father and a bystander for six minutes prior to EMS arrival. Her initial rhythm was asystole and she was given $1 \mathrm{mg}$ epinephrine and $6 \mathrm{mg}$ naloxone through an intraosseous cannula. She had return of spontaneous circulation. The ECG tracings recorded by paramedics immediately following cardiac arrest demonstrate a sinus rhythm with a QRS duration of 160 msec, which progressively narrowed over the ensuing 30 minutes. In the emergency department (ED), 44 minutes later, her QRS was $94 \mathrm{msec}$ on a 12-lead ECG. Because the QRS at arrival was normal and EMS ECGs were unavailable, benzonatate ingestion was placed lower on the differential diagnosis; however urine benzonatate concentration was $8.5 \mathrm{mcg} /$ $\mathrm{mL}$. The patient made a full recovery. A literature review demonstrated numerous cases of benzonatate overdose documenting narrow QRS complexes and a dearth of reports of wide complex rhythm.

Conclusion: Patients poisoned with benzonatate may experience rapid onset and transient QRS prolongation that may resolve prior to arrival in the ED. A normal QRS interval does not rule out benzonatate overdose.

068. Severe Complications Encountered in Massive Amlodipine Overdoses Treated with Venoarterial Extracorporeal Membrane Oxygenation

Marielle A Brenner ${ }^{1,2}$, Sarah B Puryear ${ }^{1}$, Jennifer A Acciani ${ }^{1,2}$, Mary E Wermuth $^{1,2}$

${ }^{1}$ Indiana University, Indianapolis, IN, USA. ${ }^{2}$ Indiana Poison Center, Indianapolis, IN, USA 
Background: Overdose of the dihydropyridine calcium channel blocker amlodipine can lead to cardiovascular collapse. Previous case reports of amlodipine poisoned patients treated with extracorporeal membrane oxygenation (ECMO) rarely focused on the negative outcomes of these patients. Study Aim: To describe complications of ECMO treatment in amlodipine poisoned patients.

Methods: This is a case series of two amlodipine poisoned patients who transferred to an ECMO center at the guidance of a medical toxicologist. Case one: 56-year-old female presented after ingesting $450 \mathrm{mg}$ of amlodipine. Venoarterial (VA) ECMO was started on hospital day (HOD) one for cardiogenic shock refractory to treatment with epinephrine, norepinephrine, and high dose insulin (HDI). Additional interventions included vasopressin, angiotensin II, continuous venovenous hemofiltration, and therapeutic plasma exchange. Case two: A 14-year-old female presented after intentional ingestion of $670 \mathrm{mg}$ of amlodipine. VA ECMO was started on HOD one for cardiogenic shock refractory to epinephrine, norepinephrine, and HDI. Additional interventions included intralipid emulsion prior to transfer, vasopressin, methylene blue, calcium infusion, and hydrocortisone.

Results: Case one: On HOD eight, vascular surgery was consulted for an ischemic left lower extremity which was the result of prolonged ECMO cannulation, but they were unable to intervene as she was hemodynamically unstable. After these discussions, her family advocated for comfort care measures, and she died that day. Case two: On HOD three, she had near complete decannulation resulting in loss of three to four liters of blood. She was emergently transfused a total of four units of packed red blood cells and taken to the operating room emergently for recannulation. She was decannulated on HOD five and survived neurologically intact. Conclusion: VA ECMO in the treatment of poisoned patients can result in severe complications. Medical toxicologists should be knowledgeable of both the risks and benefits of ECMO when recommending this therapy.

069. Neonatal Iatrogenic Flecainide Overdose with Only EKG Changes: A Case for Non-Intervention?

Justin A Seltzer ${ }^{1,2}$, Aaron Schneir ${ }^{1}$, Alicia B Minns ${ }^{1,2}$

${ }^{1}$ University of California, San Diego Division of Medical Toxicology, Department of Emergency Medicine, San Diego, CA, USA. ${ }^{2}$ VA San Diego Healthcare System, San Diego, CA, USA

Background: Flecainide is first line treatment for refractory neonatal supraventricular tachycardia (SVT). Recommended dosing is between $100 \mathrm{mg}$ and $200 \mathrm{mg} / \mathrm{m} 2 /$ day. Toxicity manifests QRS and QT interval prolongation but can progress to life-threatening bradycardia, dysrhythmias, and hemodynamic instability.

Methods: This is a single patient chart review. A 22-day-old, eight kg male, delivered at 36 weeks for fetal SVT presented after family noted his home flecainide was incorrectly concentrated, $20 \mathrm{mg} / \mathrm{ml}$ instead of 10 $\mathrm{mg} / \mathrm{ml}$. He was discharged four days prior on $10 \mathrm{mg}$ flecainide twice daily for refractory SVT. An EKG obtained before discharge showed a rate of $111 \mathrm{BPM}$, a PR interval of $106 \mathrm{~ms}$, a QRS interval of $80 \mathrm{~ms}$, and a QTc interval of $454 \mathrm{~ms}$. His flecainide concentration was therapeutic $(0.37 \mu \mathrm{g} / \mathrm{ml}$; therapeutic $0.20-1.00 \mu \mathrm{g} / \mathrm{ml})$ at that time. He reportedly received twice his intended dose, $20 \mathrm{mg}$ twice daily $(173.9 \mathrm{mg} / \mathrm{m} 2 /$ day; five $\mathrm{mg} / \mathrm{kg}$ /day), for eight doses. The correct dose, $10 \mathrm{mg}$, was given 2.5 hours prior to arrival. Propranolol, his other medication, was dosed appropriately.

Results: Initial vital signs, physical exam, and laboratory evaluation were unremarkable. The initial EKG showed a rate of 113 BPM, PR of $118 \mathrm{~ms}$, QRS of $138 \mathrm{~ms}$, and QTc of $513 \mathrm{~ms}$. A blood flecainide concentration obtained 6.5 hours after the last dose was $1.6 \mu \mathrm{g} / \mathrm{ml}$.Sodium bicarbonate was administered without effect. The EKG normalized by 16 hours after arrival and flecainide was restarted without complications.

Conclusions: This is a rare case of neonatal flecainide toxicity occurring within an accepted therapeutic flecainide dosing range. A similar case, reported in 2013, resulted in bradyasystolic arrest following four accidentally doubled flecainide doses. This further demonstrates flecainide's narrow therapeutic index and the increased morbidity associated with even minor dosing errors.

070. Sodium Nitrite Induced Severe Methemoglobinemia Managed with Venous-Arterial ECMO

Will R Goodrich, Morgan E Irwin-Weyant, Ryan J Cole, Avery E Michienzi, Aaron S Frey, Heather A Borek

University of Virginia, Charlottesville, Virginia, USA

Background: Sodium nitrite causes life-threatening methemoglobinemia and is available for purchase online from vendors offering lab chemicals or sold as part of suicide kits. Venous-arterial (VA) extracorporeal membrane oxygenation (ECMO) is well-described as a rescue therapy for patients in inotrope-refractory cardiogenic shock. Management of methemoglobinemia with veno-venous (VV) ECMO has previously been described; however, no reports exist to describe the use of VA ECMO for this indication.

Methods: This is a single patient chart review of a 27 -year-old female with a history of depression and post-traumatic stress disorder on fluoxetine, aripiprazole, clonazepam, and gabapentin who presented by ambulance after self-call to 911 with an intentional ingestion of sodium nitrite. Ten months prior she was treated for an ascribed accidental ingestion of sodium nitrite, which was successfully managed with methylene blue. On this presentation her initial methemoglobin level was $73.3 \%$, peaked at $86.2 \%$ despite $2 \mathrm{mg} / \mathrm{kg}$ of intravenous methylene blue in divided doses, as well as Vitamin C. However, she developed progressive spontaneous clonus attributable to methylene blue associated serotonin syndrome. Her blood pressure was initially maintained on two vasopressors, but she continued to suffer from hemodynamic instability and rising lactate. The decision was made to pursue VA ECMO and exchange transfusion. Results: She underwent ECMO cannulation by sternotomy in the emergency department two hours after ingestion with subsequent erythrocytapheresis of seven units via the ECMO circuit. Methemoglobinemia markedly improved to $10.7 \%$ seven hours after presentation. After 16 hours, she was decannulated and no longer required pressors. Methemoglobin level continued to decline to $0.7 \%$ at time of ECMO decannulation and there was no subsequent rebound. She was extubated on hospital day six and medically cleared for psychiatry on hospital day 11 .

Conclusions: In patients with methemoglobinemia complicated by cardiogenic shock, VA ECMO may support erythrocytapheresis and enable subsequent resolution of methemoglobinemia.

071. Calcium Channel Blocker Toxicity in an Under-Resourced Setting: the Importance of GI Decontamination

Muhammad Akbar Baig, Joshua Nogar

North Shore University Hospital, Manhasset, NY, USA

Background: The treatment of calcium channel blocker (CCB) toxicity requires prompt recognition, decontamination (if warranted), resuscitation, and antidotal therapies, such as high-dose insulin. However, in some practice settings, novel therapies are unavailable or are in short supply. We describe a fatality due to a large CCB overdose in a low-resource setting where gastric lavage may have been a life-saving intervention. Methods: This is a case report from Pakistan of a 22-year-old female with no previous medical history who ingested 300 tablets of amlodipine five $\mathrm{mg}$. One hour after the overdose, she presented to a healthcare facility that promptly referred her on to a higher level of care. Four hours after arrival to a tertiary facility, she was hypotensive which was refractory to intravenous fluids (IVF) \& was started on vasopressors. A bedside point of care ultrasound showed a severely depressed LV contractility. High Dose Insulin Euglycemic Therapy (HIE) was recommended but not implemented on account of a shortage of insulin. There were no ventilator beds or extracorporeal membrane oxygenation (ECMO) capabilities, and the patient suffered a peri-intubation cardiac arrest.

Results: CCBs overdose management is complicated, and a widely accepted approach involves Gi decontamination, urgent administration of 
IVF, calcium, atropine, vasopressors, and HDIT, with an addition of novel adjunct therapies based on necessity. Such multifaceted management is a high resource utilization process. However, GI decontamination represents perhaps the simplest, resource-independent intervention that can be performed to a poisoned patient.

Conclusion: $\mathrm{CCB}$ toxicity can be disastrous and under-resourced practice environments should aim to focus on GI decontamination of some kind to offset critical illness earlier on. Strategies should also be implemented for arranging medical resources or ensuring timely referrals to better equipped hospitals for continuity of care.

072. To Reverse or Not to Reverse? A Question of Coagulopathy Reversal in Warfarin Toxicity

Christopher J Watson ${ }^{1,2}$, James D Whitledge ${ }^{1}$, Michael D Simpson ${ }^{1}$, Michele M Burns ${ }^{1}$

${ }^{1}$ Boston Children's Hospital, Boston, MA, USA. ${ }^{2}$ Beth Israel Deaconess Medical Center, Boston, MA, USA

Background: Warfarin, a vitamin K epoxide reductase inhibitor, causes coagulopathy. While CHEST Guidelines for Evidence-Based Management of Anticoagulant Therapy protocolizes reversal of supratherapeutic international normalized ratio (INR) for patient's dependent on anticoagulation, practices vary for reversing warfarin-induced coagulopathy after overdose in non-warfarin dependent patients. Hypothesis: Treatment with a restrictive reversal strategy is reasonable for non-warfarin dependent patients with warfarin overdose.

Methods: This is a case report. A 15-year-old female with depression on sertraline ingested 100-200 mg of her father's warfarin in a self-harm attempt. At hour 24 post-ingestion, her INR was 2.00 and she was admitted for INR monitoring. Sertraline was held. Warfarin concentrations were trended.

Results: The INR was 5.10 at hour 60 and Vitamin K1 (VK) $2.5 \mathrm{mg}$ was given orally at hour 65 . At hour 85 , the INR peaked at 6.67 and she received a second dose of VK $2.5 \mathrm{mg}$ orally at hour 89 . At hour 203 (8.5 days), she was medically cleared with an INR of 1.31. Awaiting psychiatry placement on day 11 , she developed lower abdominal pain and diarrhea. Imaging revealed a duodenal hematoma, thought to be incidental by gastroenterology and surgery. Her symptoms resolved, and she was again medically cleared 13 days post-ingestion. Her serum warfarin concentration peaked at $19 \mathrm{mcg} / \mathrm{mL}$ at hour 46 and demonstrated first-order elimination kinetics with a 30-hour half-life.

Conclusion: This patient had evidence of coagulopathy at 24 hours but did not receive her first of two VK doses until hour 65 and ultimately had coagulopathy complicated by a duodenal hematoma and 13-day length of stay. Restrictive VK therapy for the non-warfarin dependent patient with intentional warfarin overdose may result in coagulopathy, bleeding, and significant length-of-stay. Reversal at 24 hours regardless of INR may be beneficial. Delayed peak warfarin concentrations support consideration of decontamination in late presenters.

\section{Video Laryngoscopic-Guided Gastric Intubation for Gastric} Lavage

Khizer Rizvi, Zachary Oliff, Juliana Chang, Muhammad Baig, Josh Nogar

Northshore University Hospital, Manhasset, NY, USA

Background: Gastric lavage is a method for early gastrointestinal decontamination for suspected acute toxic ingestions. The orogastric tubes are often 32 French in size or greater. Tracheal intubation is often needed prior to performing gastric lavage. The traditional method of inserting an orogastric lavage tube is blind with anatomic landmarks in the pharynx, however there can be difficulty in first pass success. Hypothesis: The use of video laryngoscopy to guide orogastric intubation will increase first pass success, decrease time of procedure, as well as decrease rate of complications in acute ingestions.

Methods: This is a single patient chart review. An eighteen-year-old female with a past medical history of depression and anxiety presented to the Emergency Department for unresponsiveness. The patient was found by her mother in her room minimally responsive. The patient had access to quetiapine $400 \mathrm{mg}$, lamotrigine $200 \mathrm{mg}$ extended release, and propranolol $20 \mathrm{mg}$, in which none of the pill bottles could be found. Time or quantity of ingestion was unknown. Patient's GCS was seven, HR 66, BP 80/40. Patient was intubated for airway protection.

Results: When placing the orogastric tube for lavage, video laryngoscopy was utilized while inserting and advancing the tube. First, the endotracheal tube was moved and secured laterally in the oropharynx. Next, with a Glidescope laryngoscope, the endotracheal tube was seen to be in place, the esophagus was identified. A 32-French orogastric tube was advanced into esophagus without complication. The orogastric tube was successfully inserted within 30 seconds, with small pill fragments and white slurry lavaged from patient.

Conclusions: The use of video laryngoscopy helps improve first pass success as well as decreases the duration and risk of complications for orogastric intubation for gastric lavage in acute toxic ingestions.

\section{Snake Bites in Diabetic Patients: A Descriptive Analysis}

Sukhshant K Atti ${ }^{1,2}$, Erin Ryan ${ }^{2}$, William Rushton ${ }^{1,2}$, Michael Beuhler, Kerollos Shaker ${ }^{4}$, Kim Aldy ${ }^{4,5}$, Sharan Campleman ${ }^{5}$, Paul Wax ${ }^{4,5}$, Jeffrey Brent ${ }^{6}$, Michelle Ruha ${ }^{7}$, On Behalf of the Toxicology Investigators Consortium (ToxIC) North American Snakebite Study Group

${ }^{1}$ University of Alabama at Birmingham, Birmingham, Alabama, USA. ${ }^{2}$ Alabama Poison Information Center, Birmingham, Alabama, USA. ${ }^{3}$ North Carolina Poison Control, Atrium Health, Charlotte, North Carolina, USA. ${ }^{4}$ University of Texas Southwestern Medical Center, Dallas, Texas, USA. ${ }^{5}$ American College of Medical Toxicology, Phoenix, Arizona, USA. ${ }^{6}$ University of Colorado, Aurora, Colorado, USA. ${ }^{7}$ Banner-University Medical Center Phoenix, Phoenix, Arizona, USA

Background: There is limited literature examining the impact of comorbidities such as diabetes on the clinical course of snake envenomations. This report summarizes characteristics of snake envenomations in patients with diabetes.

Methods: All cases reported to the Toxicology Investigators Consortium North American Snakebite Registry between 2013 and 2020 in patients with a history of diabetes were reviewed, and data on demographics, clinical effects, treatments, and outcomes were extracted.

Results: Thirty-four cases from 10 states were identified. Median age was 46.5 years (range $3-75$ years) and $67.6 \%(n=23)$ were male. The majority of patients $(\mathrm{n}=26,76.5 \%)$ had at least one additional comorbidity. Most bites involved rattlesnakes $(n=18,52.9 \%)$ or copperheads $(n=10,29.4 \%)$, and there were equal numbers of upper and lower extremity bites. The most common effects were swelling $(\mathrm{n}=32,94.1 \%)$ and ecchymosis $(\mathrm{N} 22$, $64.7 \%$ ). Hemotoxicity (platelets $<120$, fibrinogen $<170$, or PT $>15$ ) occurred in $20.6 \%(n=7)$ of patients initially and $20.6 \%(n=7)$ at follow up. Three patients $(8.82 \%)$ developed necrosis; two required procedures (debridement, incision and drainage, and/or skin graft). Hypotension was noted in five patients (14.7\%) but resolved with IV fluid resuscitation in all but one. Two patients $(5.88 \%)$ required mechanical ventilation. Antivenom was administered in $88.2 \%(n=30)$ of cases with a median total dose of 10 vials (range 4-53 vials; IQR 6-13.5 vials). Hospital length of stay was $<48$ hours in $82.4 \%(n=28)$ of patients and $<24$ hours in $41.2 \%(n=14) ; 41.2 \%(n=$ 14) were admitted to an intensive care unit. At final follow up (up to three weeks from initial bite), seven patients $(20.6 \%)$ had residual functional deficits and one (2.94\%) suffered permanent tissue loss.

Conclusion: The majority of snake envenomations in diabetes patients described in this report required treatment with antivenom and most had short hospital stays.

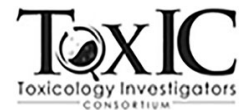

This research was performed in collaboration with the ACMT Toxicology Investigators Consortium. 
075. Pediatric Crotalid Envenomation: Critical Care Interventions, and Adverse Effects of FAB administration

Robert Goodnough ${ }^{1}$, Kelli Kulik ${ }^{2}$, Stephen A Harding ${ }^{2,3}$, Sarah Shafer ${ }^{2,3}$, Brady S Moffett ${ }^{2,3}$

${ }^{1}$ Greater San Antonio Emergency Physicians, San Antonio, TX, USA. ${ }^{2}$ Baylor College of Medicine, Houston, TX, USA. ${ }^{3}$ Texas Children's Hospital, Houston, TX, USA

Background: North American pit viper (NAPV) envenomation is a common cause of hospitalization and intensive care unit (ICU) admission. Literature demonstrates a high incidence of critical care in the admission of these patients, despite a low mortality rate and low incidence of severe adverse effects of antivenom administration, but little detail regarding specific critical care interventions.

Hypothesis: The incidence of critical care intervention or severe adverse effects of treatment is low among pediatric NAPV envenomation, who received anti-venom.

Methods: A ten-year retrospective descriptive study was conducted at Texas Children's Hospital for patients who were $\leq 18$ years-old, bitten by a NAPV, and received Crotalidae Polyvalent Immune FAB (Fab). Analyzed data were: demographics, admission level of care, medications administered, vital signs and laboratory values. Critical care interventions were epinephrine or vasoactive/inotropic medication administration, rapid sequence intubation (RSI), surgical intervention, or blood product administration. Hypotension was a systolic blood pressure $<70 \mathrm{mmHg}$ tage [years] x 2 (1-10 years-old); < $90 \mathrm{mmHg}$ (age > 10 years-old)).

Results: 255 patients were included. No mortality occurred. Median vials of Fab administered was 10, median of four doses. 5.6\% of admissions were to an ICU. Medications for hypersensitivity reactions were: IM epinephrine $(0.3 \%, \mathrm{n}=1)$, vasoactive medications $(0 \%)$, diphenhydramine $(16.7 \%, \mathrm{n}=43)$, systemic steroids $(0 \%)$. No patients underwent RSI related to $\mathrm{FAB}$ administration. One patient received fasciotomy. Hypotension was documented in $16(6.3 \%)$ patients. Two $(0.7 \%)$ of these patients were admitted to an ICU. Patients with documented hypotension underwent chart review by a toxicologist. No patient reviewed demonstrated actual hemodynamic instability or received vasoactive medications. Three patients received IV fluid bolus, but not to address hypotension. One patient displayed systemic envenomation (tachycardia and emesis).

Conclusion: The need for critical care interventions was exceedingly low among pediatric patients who received antivenom administration for NAPV. This should inform decision making to limit unnecessary ICU admissions.

076. Kounis Syndrome due to North American Pit Viper Envenomation George Warpinski, Joshua P Canning

Banner - University Medical Center, Phoenix, AZ, USA

Background: Kounis syndrome refers to acute coronary syndrome associated with mast cell activation and release of inflammatory mediators including histamine and tryptase in response to an allergic insult. It has been described following numerous drug and environmental exposures including other snake species. There are no previously reported cases of North American pit viper envenomation leading to Kounis syndrome.

Methods: This is a single patient chart review. A 39-year-old man with a history of COPD and hypertension was transported to our hospital after sustaining a rattlesnake bite to his left calf. The patient developed lightheadedness, diaphoresis, chest pain, and vomiting immediately after the bite. Pre-hospital, the patient became transiently hypotensive and was started on a norepinephrine infusion. ST segment elevation was noted on his initial rhythm strip.

Results: EKG on arrival showed inferior ST segment elevation with reciprocal ST segment depression. Cardiac catheterization showed right coronary artery vasospasm that improved with nitroglycerin. Prior to contrast administration and antivenom, he received IV corticosteroids and antihistamines for pruritis and dyspnea thought to be due to an allergic reaction from the envenomation. He received 10 vials of crotalidae immune $F\left(a b^{\prime}\right) 2$ in the catheterization suite followed by an additional eight vials for progression of swelling of the lower extremity although overall swelling was limited and never progressed beyond the knee. His prothrombin time, platelets, and fibrinogen remained normal. Initial high sensitivity troponin was $1,308 \mathrm{ng} / \mathrm{L}$ which trended down to $819 \mathrm{ng} / \mathrm{L}$ during the first hospital day. He did not receive heparin or antiplatelet agents. His echocardiogram showed a normal ejection fraction. ST segment elevation resolved by hospital day three.

Conclusion: This is a case of Kounis syndrome due to pit viper envenomation and is the first report of a North American pit viper envenomation leading to coronary vasospasm and STEMI.

077. Misguided Home Care Causing Severe Tissue Damage Following Rattlesnake Envenomation

Brian G Lewis ${ }^{1}$, Dominic Pappas ${ }^{2}$, Daniel E Brooks ${ }^{1}$

${ }^{1}$ University of Arizona College of Medicine/Banner Health Medical Toxicology Fellowship, Phoenix, AZ, USA. ${ }^{2}$ Creighton University School of Medicine/Maricopa County Emergency Medicine Residency, Phoenix, AZ, USA

Background: Rattlesnake envenomations (RSE) can result in significant clinical effects, including local edema and tissue necrosis that may be worsened with misguided self-care or delayed medical attention. We report the case of local RSE effects exacerbated by delayed presentation and misguided wound care.

Methods: This is a single chart review case report. A 15-year-old, previously healthy boy was bitten between his left third and fourth fingers by a rattlesnake. The patient applied two tourniquets to his arm and forearm for three hours. He then soaked the envenomated hand in household bleach ( $6 \%$ sodium hypochlorite) for several hours overnight. He presented to an Emergency Department (ED) 24 hours after the RSE. His initial labs included the following: platelets $201 \times 10^{9} / \mathrm{L}$, PT $11.8 \mathrm{~s}$, fibrinogen $329 \mathrm{mg} / \mathrm{dL}, \mathrm{Hgb} 15.2 \mathrm{~g} / \mathrm{L}$.

Results: The patient was splinted in elevation, received 6 vials of crotalidae polyvalent immune fab and transferred to our admitting Medical Toxicology service. In our PICU he received a total of 14 vials of crotalidae immune $\left.\mathrm{F}(\mathrm{ab})_{2}\right)_{2}$ for concern for continued local tissue toxicity and local decontamination with water. He had diminished sensation over his hand and fingers with deep grade 2 burns but normal pulse oximetry readings and waveforms on all fingers. He was transferred to the state's Burn Center. He was hospitalized for 14 days; he had multiple debridement procedures, two skin grafts, and received occupational therapy for maximal hand/finger functioning.

Conclusion: Misguided home care can lead to additional morbidity following RSE. Increased outreach to the public promoting prompt medical care and guidelines on proper field management following RSE can decrease RSE morbidity.

078. Multi-limb Snakebite Envenomations: A Case Series from a Single Poison Center

Nicholas V Titelbaum, Joseph E Carpenter

Department of Emergency Medicine, Emory University School of Medicine, Atlanta, GA, USA

Background: Approximately 7,000-8,000 people are bitten by venomous snakes in the United States annually. Little literature exists describing multi-limb venomous snakebites.

Research Question: What is the clinical course of patients sustaining multi-limb venomous snakebites?

Methods: This is a retrospective case series with data pulled from a single poison center between July and October, 2021.

Results: Three cases of multi-limb bites by suspected venomous snakes were identified. Case one: A 50-year-old man presented to the ED with bites to both hands by an Eastern copperhead, Agkistrodon contortrix. Crotalidae polyvalent immune Fab (CroFab) was administered when the 
swelling reached one of his elbows. He received a total of 12 vials of CroFab. The swelling progressed halfway between the elbow and shoulder on one extremity and to the mid-forearm on the other. He never developed laboratory or clinical evidence of coagulopathy and was discharged on hospital day four. Case two: A 30-year-old man presented to the ED with bites to both feet by an Eastern copperhead. Exam revealed multiple puncture wounds on bilateral mid-foot areas. Swelling extended to the ankles. He never developed laboratory or clinical evidence of coagulopathy, did not receive antivenom, and was discharged after 12 hours of observation. Case three: A 13-year-old male presented to the ED with bites to both hands after picking up a suspected Eastern copperhead. Exam revealed multiple puncture marks on the fingers without swelling, erythema, or ecchymosis. He never developed laboratory or clinical evidence of coagulopathy, did not receive anti-venom, and was discharged after six hours of observation.

Conclusion: A review of recent cases of multi-limb snakebites reported to a single poison center showed one case that required anti-venom and four days of hospitalization, one patient who developed edema but did not require anti-venom, and one patient who was asymptomatic after six hours of observation.

079. Exotic Envenomation by an African Bush Viper: A Case Report Alexandra M Amaducci, Derek J Fikse, Andrew L Koons, Kenneth D Katz

Lehigh Valley Health Network-USF Morsani College of Medicine, Allentown, Pennsylvania, USA

Background: Atheris squamigera is an African venomous snake. US envenomation is exceedingly rare with only two previous cases reported. A 26-year-old exotic pet owner developed pain and swelling on his left foot following an Atheris squamigera bite.

Case Report: A 26-year-old man with no past medical history presented to the Emergency Department (ED) for evaluation of left foot pain and swelling following a snakebite. While transferring an Atheris squamigera snake between containers, he was bitten twice on the dorsum of his left foot. In the ED, vital signs were: BP 112/80 $\mathrm{mmHg}$, HR $77 \mathrm{bpm}, \mathrm{T}$ $98.5^{\circ} \mathrm{F}, \mathrm{RR} 16 \mathrm{bpm}, 02$ saturation $99 \%$. Physical exam showed puncture wounds on dorsum of left foot with surrounding erythema, ecchymosis, and edema. Abnormal labs included: initial D-dimer $1.06[<0.50 \mathrm{ug} / \mathrm{mL}]$, WBC 12.58 [4.0-10.5 K/cmm], absolute neutrophils 9.15 [1.8-7.8 $\mathrm{K} / \mathrm{cmm}$, fibrinogen 121 [180-500 $\mathrm{mg} / \mathrm{dL}]$ and subsequent D-dimer > $20(<0.50 \mathrm{ug} / \mathrm{mL})$. Additional laboratory testing was within normal limits. After consultation with a medical toxicologist, he was transferred to a tertiary care hospital. The physician and primary investigator of Miami-Dade Fire Rescue Venom One was consulted to obtain antivenom if needed. Signs and symptoms remained local and mild, not requiring anti-venom, and the patient was discharged on hospital day two.

Discussion: The findings of swelling, ecchymosis, erythema, in addition to the mild lab abnormalities, indicated the patient was envenomated. An early indicator of envenomation is neutrophilic leukocytosis. Currently, no specific antivenom exists, but utilization of antivenoms of similar species has been described. The patient's mild presentation did not require antivenom administration, but access to antivenom was established if the envenomation progressed. Two US Atheris squamigera envenomations have been reported - only one required antivenom.

Conclusion: Physicians must be aware of exotic snake bites and the possibility of procuring antivenoms for severe envenomation.

080. Geographic Distribution of Calls to New Jersey Poison Center regarding Latrodectus Envenomation

Noah G Berland, Tuyet-anh Nguyen, Bruce Ruck, Mehruba Parris, Diane Calello

Rutgers NJMS, Newark, NJ, USA

Background: Latrodectus spp. or Widow spiders are considered in popular culture to be the deadliest spiders in the United States. This perception may incite fear and generate calls to poison control centers regarding possible envenomation. New Jersey (NJ) is known to be home to two Latrodectus spp. - L. Mactans and L. Variolus. Mapping origination locations of calls placed to the poison center can help in risk assessment of true envenomation by Lactrodectus spiders.

Methods: We performed a single poison control center retrospective chart review using ToxiCALL ${ }^{\circledR}$ (Computer Automation Systems, Inc. Aurora, CO, version 4.7.40) and the query for AAPCC sub generic code 194000 for Latrodectus Species, for suspected human exposures from Jan 2011 - Sept 2021, and generated a de-identified report by county of caller for all 21 counties in NJ. We used a GIS software package ArcGISPro (Environmental Research institute, Redlands, California, version 2.8) to generate a county level heat map normalized to population.

Results: Over the 129-month study period we identified 59 calls for potential human exposures, averaging 5.5 calls a year. All but one county in NJ had originating calls regarding Latrodectus spp. Four counties in the southern NJ - Salem, Cumberland, Cape May, and Atlantic have the highest call volume when normalized by population, accounting for 30 out of $59(50.8 \%)$ of calls in NJ. Ocean county, which is contiguous with the four counties, had the fifth highest call volume when normalized by population and the second most (12) overall. These five contiguous counties account for $71 \%$ of calls regarding potential human exposures to Latrodectus spp.

Conclusion: Latrodectus envenomation is uncommon in NJ but does occur. We report a geographic pattern of southern and coastal counties which may reflect the underlying habits of these spiders and likelihood of envenomation by region.

081. Lead Encephalopathy Treated with Dimercaprol, Edetate Calcium Disodium and Continuous Renal Replacement Therapy in a Patient with End-Stage Renal Disease

James D Whitledge ${ }^{1,2}$, Hilary J Branch ${ }^{3}$, Michael J Germain ${ }^{3}$, Stephanie A Hibbard $^{3}$, Rose H Goldman ${ }^{4,2}$, Alan D Woolf ${ }^{1,2}$, Michele M Burns ${ }^{1,2}$ ${ }^{1}$ Boston Children's Hospital, Boston, MA, USA. ${ }^{2}$ Harvard Medical Toxicology Fellowship, Boston, MA, USA. ${ }^{3}$ Baystate Medical Center, Springfield, MA, USA. ${ }^{4}$ Cambridge Health Alliance, Cambridge, MA, USA

Background: Treatment of lead toxicity with chelating agents relies on renal excretion of lead-chelator complexes. However, in patients with poor renal function requiring dialysis, data on optimal dialysis and chelation regimens is limited.

Methods: This is a case report. A 21-year-old female with end-stage renal disease and pica involving dirt outside her home presented to hospital with encephalopathy and venous blood lead level (BLL) of $136 \mathrm{mcg} / \mathrm{dL}$. Abdominal imaging showed radiopaque foreign bodies which were decontaminated with whole bowel irrigation. The patient was admitted to the ICU. Dimercaprol $250 \mathrm{mg}$ was given intramuscularly every four hours for four days, then only intermittently for two days because of patient discomfort. Edetate calcium disodium (EDTA) was given intravenously approximately every four hours for three days concurrently. EDTA drug shortages required inter-hospital sharing and drug compounding, and variable dosages. Continuous renal replacement therapy (CRRT) was performed throughout chelation because of concern for exacerbating encephalopathy with intermittent hemodialysis of chelated lead. Daily BLLs, zinc protoporphyrin level (ZPP), and dialysate fluid lead levels were obtained.

Results: BLLs down trended from $136 \mathrm{mcg} / \mathrm{dL}$ to $15 \mathrm{mcg} / \mathrm{dL}$ and chelation was discontinued. However, levels rebounded to $74 \mathrm{mcg} /$ dL after redistribution and recurrent pica, necessitating further chelation. Dialysate fluid lead levels were $138 \mathrm{mcg} / \mathrm{L}$ after dimercaprol and $264 \mathrm{mcg} / \mathrm{L}$ after concomitant dimercaprol and EDTA, indicating effective and additive extracorporeal extraction. ZPP level was elevated to $151 \mathrm{mcg} / \mathrm{dL}$, suggesting chronic exposure. Soil from the patient's home contained elevated lead at $774 \mathrm{ppm}$. Mental status returned to baseline prior to discharge. Follow-up BLL seven weeks later was $38.7 \mathrm{mcg} / \mathrm{dL}$. 
Conclusion: This case documents successful treatment of life-threatening lead encephalopathy in a young adult with chronic renal failure. Severe lead toxicity in dialysis patients may be effectively treated with dimercaprol and EDTA chelation and CRRT.

082. Induction of Labor for Source Removal as Opposed to Maternal Chelation in a Term-Infant with Severe Gestational Lead Exposure Sarah Mahonski ${ }^{1}$, Sanjay Mohan ${ }^{1}$, Emily T Cohen ${ }^{1}$, Silas Smith ${ }^{1}$, Mark $\mathrm{K} \mathrm{Su}^{2}$

${ }^{1}$ Division of Medical Toxicology, Ronald O. Perelman Department of Emergency Medicine, NYU Grossman School of Medicine, New York, NY, USA. ${ }^{2}$ New York City Poison Control Center, Department of Health and Mental Hygiene, New York, NY, USA

Background: Gestational lead exposures can result in teratogenicity and long-term developmental issues. Current recommendations for pregnant women with a blood lead level (BLL) $>45 \mathrm{mcg} / \mathrm{dL}$ include maternal chelation. Breastfeeding is not recommended until maternal BLL is < $40 \mathrm{mcg} / \mathrm{dL}$. We present a case of severe gestational lead exposure treated with induction of labor in a term infant.

Methods: This is a single patient chart review. A 22-year-old woman, G2P1001 at 38 weeks and one day presented to the hospital with an outside hospital BLL of $53 \mathrm{mcg} / \mathrm{dL}$. After discussing chelation versus induction of labor, she underwent immediate induction of labor overnight and delivered a well appearing, 3510-gram infant with APGAR scores of nine and nine the following day. The infant was empirically treated with succimer $350 \mathrm{mg} / \mathrm{m}^{2}$ per day pending BLLs. Succimer was discontinued when infant blood lead concentration returned $<45 \mathrm{mcg} / \mathrm{dL}$.

Results: A maternal BLL at the time of induction initiation rose to 70 $\mathrm{mcg} / \mathrm{dL}$ with a simultaneous hemoglobin of $5.8 \mathrm{mg} / \mathrm{dL}$. The mother was transfused with two units of packed red blood cells. A maternal BLL at the time of delivery decreased to $43 \mathrm{mcg} / \mathrm{dL}$. Infant cord blood lead was $41 \mathrm{mcg} / \mathrm{dL}$. Maternal breast milk lead concentration was sent and is pending. Succimer was continued in the infant for five days and discontinued when infant BLL returned at $41 \mathrm{mcg} / \mathrm{dL}$. The infant's BLL rebounded to $33 \mathrm{mcg} / \mathrm{dL}$ after cessation of succimer. Breastfeeding was initiated after the mother's BLL was confirmed to be under $40 \mathrm{mcg} / \mathrm{dL}$. Conclusions: Faced with rising lead concentrations, induction of labor in term pregnancy presents an alternative option to decrease fetal lead exposure and obviates the need for antidotal therapy that is expensive, in short supply and potentially associated with adverse effects.

083. Investigation of Autopsy Results in Cadavers With Lead Poisoning in Tehran Legal Medicine Center Over 10 Years

Fares Najari, Babak Mostafazadeh

Shahid Beheshti University of Medical Sciences, Tehran, Iran

Background: Due to the rapid pace of industrialization and the high prevalence of addiction, toxicity caused by heavy metals, especially lead, has become one of the major health problems associated with high morbidity and mortality. Therefore, reliable information is critical to manage this condition.

Methods: This descriptive cross-sectional study was performed on 30 cadavers, suspected of lead poisoning. The hospital records and the results of anatomical investigations were studied. Data was collected in a researcher-made questionnaire and analyzed in SPSS Version 22.

Results: The results showed a significant correlation between the pathological results of pulmonary autopsy and the duration of drug use $(\mathrm{p}=$ $0.01)$. Also, the pathological results of cardiac autopsy had significant correlations with age $(\mathrm{p}=0.006)$ and blood lead level $(\mathrm{p}=0.03)$. Moreover, significant correlations were found between the pathological results of liver autopsy and age $(p=0.00)$, between the pathological results of brain autopsy and the route of drug administration $(p=0.01)$, and between the pathological results of kidney autopsy and age $(\mathrm{p}=$ $0.00)$. Most pathological changes were observed in the brain and kidney tissues.
Conclusion: Lead poisoning does not cause any specific pathological changes in the liver, heart, brain, lung, or kidney tissues; however, these non-specific changes, alone or together, can lead to death.

084. Mercury Poisoning Associated with Immune-Mediated Encephalitis Michael Semple ${ }^{1}$, Ari Filip ${ }^{1}$, Evan Schwarz ${ }^{1}$, David Liss ${ }^{1}$, Michael Mullins $^{1}$, Kevin Baumgartner ${ }^{1}$, Jason Devgun ${ }^{1}$, Arrianna Thompson ${ }^{2}$

${ }^{1}$ Washington University School of Medicine, St. Louis, MO, USA. ${ }^{2}$ Barnes Jewish Hospital, St. Louis, MO, USA

Background: Low-grade, chronic elemental mercury exposures occur infrequently and are challenging to diagnose. Autoimmune manifestations are rarely associated with mercury toxicity.

Hypothesis: Elemental mercury toxicity can cause autoimmune encephalitis.

Methods: This is a single patient chart review. An 11-year-old and her family presented with upper respiratory symptoms, fever, and an erythematous convalescent rash. These improved, but the patient subsequently developed chronic polyarthralgia, back pain, abdominal pain, and headache. Her pediatrician prescribed steroids, but when she failed to improve, she was admitted for further diagnostics. During her 17 day hospital stay, her pain proved difficult to control despite the use of multiple medications and she had a single new onset seizure. On presentation and throughout her admission she remained persistently tachycardic and hypertensive. Cardiology, rheumatology, palliative/complex care, neurology, nephrology, neurosurgery and endocrinology were consulted without improvement in clinical course or a definitive diagnosis. Heavy metal testing was eventually ordered, after resulting, the patient confirmed mercury exposure; mercury was spilled in a sibling's room and vacuumed almost daily.

Results: A thorough laboratory evaluation demonstrated a slightly elevated T4 of $1.77 \mathrm{ng} / \mathrm{dL}$ with normal TSH, elevated CSF protein of 80.2 $\mathrm{mg} / \mathrm{dL}$, elevated spot and $24 \mathrm{~h}$ metanephrines without evidence of adrenal mass on MRI, CT and MIBG imaging. A 24-hour heavy metal testing demonstrated a mercury concentration of $214 \mathrm{mcg} / \mathrm{g} \mathrm{Cr}$, and she began chelation with succimer. The day after starting chelation, lumbar puncture results from earlier in the hospitalization returned positive for CASPR2 antibodies suggesting a neuro-autoimmune encephalitis. Treatment with rituximab is scheduled.

Conclusion: Elemental mercury exposures may result in auto-immune encephalitis. This may explain why our patient's symptoms were more severe and persistent than those of other family members living in the same home.

\section{Hiding From the Drug Screen: A Case of Extensive Nitrous Oxide Misuse \\ Avery Michienzi, Lauren Smaltz, Christopher Holstege \\ University of Virginia, Charlottesville, VA, USA}

Background: Nitrous oxide (NO) is an inhalant used for its euphoric effects. NO is not tested for on employer urine drug screens (UDS) so use can go undetected.

Methods: This is a single patient chart review of a 48-year-old male commercial airline pilot who was evaluated in a Medical Toxicology clinic due to history of NO misuse. He admitted to using NO for years and not other substances to ensure that he passed employer drug screens. During the COVID-19 pandemic, he was using up to 1,200 eight gram NO canisters daily. He developed paresthesias, progressive leg weakness, ataxia and cognitive decline. He was brought to the hospital after being found altered in his garage. Upon referral to the Medical Toxicology clinic, he had not used NO for three months and had been taking vitamin B12. Symptoms had improved, but he still had extremity paresthesias, memory difficulties, and required a cane to walk. During his hospitalization, the NO misuse was reported to the Federal Aviation Administration (FAA) and he was no longer allowed to pilot commercial airlines. 
Results: During hospitalization, MRI imaging of the brain showed atrophy from chronic toxic metabolic encephalopathy. MRI of the spine did not show abnormalities. Labs did not show significant vitamin B12 deficiency as the patient was supplementing B12 prior to hospitalization.

Conclusion: Random drug testing of airline pilots is required by the FAA and the UDS tests for marijuana, cocaine, amphetamines, opioids and phencyclidine. Negative drug screens may give an employer a false sense of security that a pilot is not using/misusing substances as these UDS do not pick up numerous abused substances, including inhalants. This case illustrates the dangers in relying solely on the UDS as this patient was misusing nitrous oxide for decades negatively impacted his ability to safely fly.

\section{Sniffing Skeletal Fluorosis: A Rare Manifestation of Difluoroethane} Inhalant Abuse

Derek J Fikse, Danny Le, Andrew H Miller, Ryan M Surmaitis

Lehigh Valley Health Network, Department of Emergency and Hospital

Medicine/USF Morsani School of Medicine, Allentown, PA, USA

Background: Inhalants are substances of abuse commonly used for the desired effect of euphoria. The term "dusting" refers to the inhalation of compressed air cleaners. Skeletal fluorosis is a bone disorder caused by excessive fluoride deposition. It is typically associated with industrial or drinking water sources and is rare in developed countries. We report a case of skeletal fluorosis from chronic inhalation of 1,1 difluoroethane.

Case: A 33-year-old male with inhalant use disorder presented to the emergency department with depression and suicidal ideation. Physical exam displayed prominent bilateral hand swelling and long bone deformities. Upon further questioning the patient reported five years of inhaling "surf-onn. ${ }^{(}$computer dust-off" (1,1 difluoroethane) in increasing frequencies and amounts. Over the past six months he noted significant bilateral hand pain and deformity that was interfering with his daily functions. Radiographs of his bilateral hands, forearms, lower extremities, and chest demonstrated diffuse periosteal new bone formation and sclerosis. The patient was diagnosed with skeletal fluorosis after other rheumatologic etiologies were ruled out. Diagnostic testing demonstrated a urine fluoride $64.5 \mathrm{mg} / \mathrm{L}($ ref $<3$ ) and serum fluoride $1.8 \mathrm{mg} / \mathrm{L}($ ref $<0.13)$. Cessation of inhalant use was advised, and the patient was provided with vitamin supplementation (C, D, E, and calcium).

Discussion: Inhalant use is commonly associated with central nervous system effects and cardiac dysrhythmias. Skeletal fluorosis from inhalation use is rarely reported in the literature. Cessation is needed to prevent further fluoride bony deposition. Recovery is prolonged and may take 812 years. Vitamin C, vitamin D, and calcium may aid in bone repair; vitamin $\mathrm{E}$ and methionine may reduce bone accumulation of fluoride. Conclusion: Clinicians should be aware of the potential for skeletal fluorosis in patients with chronic inhalant use. Patients with inhalant use disorder should be counseled regarding this rare but debilitating adverse effect.

087. Survival After Deliberate Self-Poisoning with Hydrogen Sulfide Gas with Delayed Myocardial Injury and Rhabdomyolysis

Pioma Wijeyesakere $^{1}$, Susana Benavente ${ }^{1}$, Andis Graudins ${ }^{1,2}$

${ }^{1}$ Monash Toxicology Unit, Monash Health, Melbourne, Victoria,

Australia. ${ }^{2}$ Monash University, Melbourne, Victoria, Australia

Background: Hydrogen Sulfide (H2S) gas exposure results in generalized inhibition of cellular respiration and sudden death, like cyanide. We report survival after $\mathrm{H} 2 \mathrm{~S}$ deliberate-self-poisoning, exhibiting reversible myocardial injury and delayed rhabdomyolysis.

Method: A single case-report of a 40-year-old male attempting suicide in his car by mixing 400-500 mL, each, of lime sulfide and 32\% hydrochloric acid. He noticed an immediate rotten egg smell and suddenly lost consciousness. Eight-hours later, he awoke and called paramedics.

Results: Assessment in emergency department; GCS-15, pulse 88-bpm, BP 144/88, SpO2 100\%. Examination revealed tongue bite marks, suggesting recent seizure. Chest was clear. Neurologic exam normal. Initial ECG: sinus tachycardia without ischemic changes. Troponin-I 36ng/L $(\mathrm{N}<20 \mathrm{ng} / \mathrm{L})$, ALT 54U/L $(\mathrm{N}<40 \mathrm{U} / \mathrm{L})$. Cranial-CT was normal. Admission echocardiography revealed localized right-ventricular (RV) dilatation and wall hypokinesis with normal LV-function. Supplemental oxygen was administered for 24-hours (FiO2 50\%). Sinus tachycardia, exertional dyspnea, orthopnea, and pedal oedema developed over threedays and gradually improved over the next two-weeks. CT-pulmonaryangiography excluded thromboembolism. RV-function normalized on serial echocardiograms over the next week. Treatment with furosemide, ramipril and amlodipine was initiated. Creatinine kinase peaked $(12,685$ $\mathrm{U} / \mathrm{L})$ on day-5 ( $<200 \mathrm{U} / \mathrm{L})$ and Troponin-I on day seven $(5,073 \mathrm{ng} / \mathrm{L})$. Serial ECGs demonstrated: PR-segment elevation in aVR and generalized ST-segment elevation, suggesting myopericarditis. CT-coronary angiogram showed normal coronary arteries. Aspirin and colchicine were added to his treatment. The patient was transferred to the mental health ward on day-14. At six-week follow-up, dyspnea had resolved complete$\mathrm{ly}$, and cognition was normal. The calculated volume of $\mathrm{H} 2 \mathrm{~S}$ released in a medium-sized car cabin would have resulted in a lethal H2S concentration of $>4,000 \mathrm{ppm}$.

Conclusion: Generation of $\mathrm{H} 2 \mathrm{~S}$ in an enclosed space, is a well-described suicide method. Survival with full recovery is uncommon. This case parallels previous reports of survivors with reversible, delayed myocardial injury and rhabdomyolysis. We also noted subsequent development of myopericarditis.

088. Therapeutic Bronchial-Alveolar Lavage Treatment of Smoke Inhalation Injuries in the Emergency Department

Joseph Liu ${ }^{1}$, Vincent Nguyen ${ }^{2}$, Wells Brambl ${ }^{3,1}$

${ }^{1}$ Maimonides Medical Center, Brooklyn, NY, USA. ${ }^{2}$ Jacobi Medical Center, Bronx, NY, USA. ${ }^{3}$ Northwell Health: Long Island Jewish, Glen Oaks, NY, USA

Background: Fiberoptic bronchoscopy is diagnostic for detection and evaluation of smoke inhalation injury (SII) in burn patients. SII treatment is traditionally limited to supportive care, treatment of carbon monoxide, and empiric treatment for cyanide. Therapeutic bronchoscopy is an adjunct to treatment of SII, with some studies showing that early initiation of therapeutic bronchoscopy may lead to improved outcomes: decreased morbidity, days on mechanical ventilation, and hospital length of stay. A putative mechanism is that early removal of carbonaceous soot decreases the inflammatory response.

Hypothesis: Therapeutic bronchoscopy for SII initiated in the emergency department (ED) may improve patient outcomes.

Methods: This is a case report of a patient with carbon monoxide poisoning, respiratory failure, and SII who we saw in the ED of an urban tertiary care facility. We performed therapeutic bronchoscopy with normal saline lavage to remove carbonaceous soot and airway-occluding secretions.

Results: A 64-year-old male presented to the ED after being found unresponsive in an apartment fire. His O2 sat on $100 \%$ bag valve mask ventilation was $94 \%$. He was obtunded and had extensive soot in the nares and oropharynx. The patient was intubated and had laryngeal edema on video laryngoscopy. The initial carboxyhemoglobin level was $26.7 \%$. Bronchoscopy was performed after intubation, which showed extensive carbonaceous soot and erythema in all segmental bronchi. The total volume of lavage was approximately $1 \mathrm{~L}$ (see figure). The patient was transferred for emergent hyperbaric oxygen treatment (HBO), and the patient underwent one session of HBO. Repeat bronchoscopy showed minimal soot in the airways. He was extubated on hospital day (HD) 15 and discharged to rehab on HD 22.

Conclusion: Therapeutic bronchoscopy in the emergency department may improve outcomes for SII. The risks to early bronchial alveolar lavage are minimal if the patient is already intubated.

089. A Case of a Diethylene Glycol Ingestion Treated with Fomepizole and its Proposed Elimination Kinetics 
Emily A Kiernan ${ }^{1,2}$, Andres Guzman-Soto ${ }^{1,2}$, Kyle Suen ${ }^{1,2}$, Prem Menon $^{1}$, Michael Zdradzinski ${ }^{1}$, Jonathan De Olano ${ }^{1,2}$

${ }^{1}$ Emory University School of Medicine, Atlanta, GA, USA. ${ }^{2}$ Georgia Poison Center, Atlanta, GA, USA

Background: Diethylene glycol (DEG) is a clear liquid found in brake fluid and antifreeze. Human renal and neurologic toxicity has been reported after inappropriate exposures. DEG produces intoxication and is metabolized by alcohol dehydrogenase (ADH) to toxic compounds: 2hydroxyethoxyacetic acid (HEAA) and diglycolic acid (DGA). Fomepizole is a competitive antagonist of $\mathrm{ADH}$ and can prevent formation of HEAA and DGA. Little is known about human pharmacokinetics of DEG while being treated with fomepizole.

Methods: This is a case report of a 35-year-old male with a history of depression who presented to the Emergency Department (ED) within three-hours of ingestion of 10-ounces of brake fluid containing 10-20\% DEG. Physical examination revealed inebriation. Labs from a psychiatric visit the day before showed: $\mathrm{HCO} 327$, creatinine 0.8 , and, within three hours after ingestion of DEG, labs showed: $\mathrm{HCO} 3$ 17, creatinine 1.1, lactic acid 8.3, ethanol $<10$, arterial pH 7.32, pCO2 34. We obtained serial serum chemistries, osmolarities, and DEG concentrations on arrival to the ED (0 hours), and at 6.9, 15.7, and 20.7 hours. Fomepizole 15 $\mathrm{mg} / \mathrm{kg}$ and $10 \mathrm{mg} / \mathrm{kg}$ was administered at 2.6 and 27.8 hours after arrival, respectively.

Results: The elimination half-life of DEG was calculated to be 13.95 hours while on fomepizole. The patient's HCO3 while on fomepizole was: $19,21,22$. After fomepizole discontinuation, his levels improved to normal. The creatinine while on fomepizole was: 1, 1, 0.8. After fomepizole discontinuation, his creatinine remained normal until hospital discharge on hospital day seven.

Conclusion: This is the first report of elimination kinetics in a human DEG ingestion treated with fomepizole. The patient received fomepizole and did not develop acidosis, renal failure, or neurotoxicity.

090. Throwing the Baby Out with the Antifreeze: Is Urine Fluorescence Useless?

Jason B Hack ${ }^{1}$, Natalija M Farrell ${ }^{2}$, Jordan K Wolfe ${ }^{3}$

${ }^{1}$ East Carolina University, Greenville, NC, USA. ${ }^{2}$ Boston Medical Center, Boston, MA, USA. ${ }^{3}$ Saint Francis Hospital, Tulsa, OK, USA

Background: Timely diagnosis of unwitnessed ethylene glycol (EG) ingestions is problematic. The ability to obtain EG levels is inconsistent, osmolar and anion gaps depend upon timing and equation, and finding urine crystals is unreliable. We present an illustrated case of an EG poisoned patient whose fluorescent urine assisted in diagnosis and expediting treatment.

Case: A 32-year-old male presented 30 minutes after claiming to drink $1 / 2$ gallon of antifreeze plus a plastic knife and two ballpoint pens. Physical examination revealed an awake, tachycardic male with epigastric tenderness. Laboratory data revealed normal renal function $(\mathrm{Cr} 0.92 \mathrm{mg} / \mathrm{dL}$, BUN $10 \mathrm{mg} / \mathrm{dL}$ ), osmol gap 24 (normal range -14 to +10 ), anion gap 13 (normal range 3-13), normal appearing urine without crystals. Fomepizole $15 \mathrm{mg} / \mathrm{kg}$ IV was given. We compared the bedside physician's urine with the patient's using a Wood's lamp (in urinal and specimen cup). Patient's sample was brightly fluorescent, supporting diagnosis of poisoning (images). Subsequent EG levels returned $130 \mathrm{mg} / \mathrm{dL}$. He was admitted to medical ICU for continued fomepizole therapy. His EG levels fell to undetectable and renal function remained normal. Endoscopy removed gastric foreign bodies. He was transferred to psychiatry and ultimately discharged without sequalae.

Discussion: Current literature suggests and dismisses urine fluorescence as a cryptic EG poisoning clue. Research reports $60 \%$ of patients had urine fluorescence after ingesting EG, while other work cast doubt on accurate EG screening.

Conclusion: Diagnosis of EG poisoned patients may be confounded by delayed return of definitive levels, unreliable osmol gaps and inconsistent urinary crystals. Although experimentally tenuous, in resource poor environments, diagnosis and treatment decisions may be helped by detecting strongly positive fluorescent urine in the setting of a suspected recent ingestion of a fluorescein-containing EG product.

091. Toxicokinetic Clearance Parameters of Ethylene Glycol Poisoning in Pregnancy

Matthew S Correia ${ }^{1,2}$, Courtney Temple ${ }^{1,2}$, Robert G Hendrickson ${ }^{1,2}$

${ }^{1}$ Oregon Health and Science University, Portland, OR, USA. ${ }^{2}$ Oregon Poison Center, Portland, OR, USA

Background: Ethylene glycol (EG) is an odorless, colorless, sweet tasting alcohol derivative frequently found in radiator fluid and de-icing solutions. Exposures in adults generally occur in the setting of intentional ingestions. It is renally excreted unchanged or metabolized via alcohol and aldehyde dehydrogenases into glycolic acid. Half-life after treatment with fomepizole is 14-17 hours in healthy individuals. There are no prior reports of the toxicokinetic clearance parameters of EG in pregnant patients after treatment with fomepizole.

Case: A 28-year-old at 15 weeks gestational age presented to the hospital after intentional ingestion of approximately 10 ounces of antifreeze. She was well appearing and reportedly not clinically intoxicated upon arrival. Vitals were notable as follows: pulse 120 beats per minute, blood pressure $136 / 88 \mathrm{mmHg}$, respiratory rate 16 breaths per minute, oxygen saturation $100 \%$ on room air and afebrile. Initial EG concentration was $263 \mathrm{mg} / \mathrm{dL}$. She was expeditiously started on fomepizole with repeat dosing every twelve hours for three days. She remained asymptomatic and did not develop a metabolic acidosis. Hemodialysis was not available. Her creatinine on arrival was $0.7 \mathrm{mg} / \mathrm{dL}$ and down trended to $0.48 \mathrm{mg} / \mathrm{dL}$. EG concentration was $47 \mathrm{mg} / \mathrm{dL}$ and $12 \mathrm{mg} / \mathrm{dL}$ at 28.8 and 52.2 hours post ingestion, respectively. Fomepizole was discontinued after three days.

Discussion: Elimination of EG after fomepizole administration followed first order kinetics with a half-life of 11.75 hours. The square of the coefficient of correlation for the exponential regression was one. This elimination half-life is notably less than that previously reported in otherwise healthy patients. However, this increase in clearance is not unexpected. Physiologic changes in pregnancy include increased glomerular filtration rate up to $50 \%$ in the first trimester.

Conclusion: We report a case of EG and toxicokinetic clearance parameters in a first-trimester pregnant woman successfully treated with solely fomepizole.

092. Symptomatic Diethylene Glycol Ingestion Successfully Treated with Fomepizole Monotherapy

Justin A Seltzer ${ }^{1,2}$, Bryan Corbett ${ }^{1}$, Daniel R Lasoff ${ }^{1,2}$, Richard F Clark ${ }^{1}$ ${ }^{1}$ University of California, San Diego Division of Medical Toxicology, Department of Emergency Medicine, San Diego, CA, USA. ${ }^{2}$ VA San Diego Healthcare System, San Diego, CA, USA

Background: Diethylene glycol (DEG) is an industrial solvent with many uses including brake fluids. This chemical has also caused mass poisonings when used as an inappropriate substitute for propylene glycol or glycerine. However, significant intentional ingestions are rare and not well reported. Like other toxic alcohols, animal studies have shown DEG is metabolized by alcohol dehydrogenase and aldehyde dehydrogenase. Its toxicity is thought to be mediated by the resulting metabolites. Fomepizole, a potent alcohol dehydrogenase inhibitor, is used to prevent metabolite formation following other toxic alcohol exposures. Fomepizole is broadly recommended for DEG poisoning, though supporting clinical evidence is very limited.

Methods: This is a single patient chart review. A 31-yr-old male presented after ingestion of DEG containing brake fluid and hydrocarbon containing "octane booster."

Results: The patient was noted to be clinically intoxicated with a mildly elevated anion gap metabolic acidosis and no osmolar gap. DEG level was later found to be elevated to $8 \mathrm{mg} / \mathrm{dl}$, consistent with his ingestion. He 
was treated with fomepizole alone, with resolution of metabolic acidosis and clinical findings over the next two days. At 52 days follow up, the patient reported no delayed neurologic sequelae.

Conclusions: Our case is one of the first to document successful treatment of verified DEG poisoning with fomepizole alone, providing meaningful clinical evidence in support of fomepizole for this indication. Consistent with other toxic alcohols, patients with DEG poisoning may benefit from empiric fomepizole administration, especially if presenting soon after exposure.

\section{Chronic Methanol Toxicity Through Topical and Inhalational} Routes Presenting as Vision Loss

Christianne V Mojica ${ }^{1}$, Esteban A Pasol ${ }^{1}$, Mercedes D Dizon ${ }^{1}$, Wenceslao A Kiat ${ }^{2}$, Timothy Reynold U Lim ${ }^{1}$, Jacqueline C Dominguez ${ }^{1}$, Vincent V Valencia ${ }^{1}$, Bernardo Joaquin P Tuaño ${ }^{1}$

${ }^{1}$ St. Luke's Medical Center, Quezon, NCR, Philippines. ${ }^{2}$ St. Luke's Medical Center, Quezon, NCR, Philippines

Background: Methanol intoxication can cause irreversible neurologic sequelae if unrecognized and untreated. Ingestion is the most common form of toxicity; however, dermal and inhalation exposures likewise occur but are documented rarely. While acute intoxication is commonly encountered, chronic exposure to methanol should also be highlighted.

Case Report: We report a case of a 57-year-old female presenting in the emergency room with progressive dyspnea, metabolic acidosis with high anion gap, and metabolic encephalopathy. After emergency hemodialysis, the patient complained of vision loss on both eyes. Initial non-contrast cranial magnetic resonance imaging (MRI) revealed restricted diffusion of the intraorbital segment of both optic nerves. A thorough history revealed that she was applying a clear colorless liquid bought online all over her body for alleged pruritus for more than a year. The syndrome of metabolic acidosis with high anion gap, metabolic encephalopathy, vision loss, and laboratory findings led us to suspect a diagnosis of chronic methanol poisoning with an acute component. The liquid in question was sent for chemical analysis and results showed that it consisted of 95.5\% Methanol.

Conclusion: This case highlights the need for high index of clinical suspicion for methanol toxicity in the absence of oral consumption, the complications of chronic form of methanol intoxication, and the uncommon radiologic finding seen in diffusion-weighted imaging (DWI).

094. Ambulatory Alcohol Withdrawal Management with Telemedicine During the COVID-19 Pandemic: Protocol and Application in a Case Series

Matthew R Dernbach ${ }^{1}$, Mayra R Beltran ${ }^{2}$, Timothy J Wiegand ${ }^{3}$

${ }^{1}$ Medical University of South Carolina, Charleston, SC, USA. ${ }^{2}$ Huther

Doyle Chemical Dependency Treatment Program, Rochester, NY, USA.

${ }^{3}$ University of Rochester School of Medicine \& Dentistry, Rochester, NY, USA

Background: The COVID-19 pandemic is associated with higher rates of Alcohol Use Disorder (AUD), and has impacted access to detoxification and inpatient levels of care. Consequently, telemedicine-based Ambulatory Alcohol Withdrawal (AAW) management is increasingly relevant. Hypothesis: Telemedicine-based AAW can be safely and effectively utilized in appropriate patients.

Methods: This is a retrospective three patient chart review during the COVID-19 pandemic. Telephone-based patient screening included: PAWSS, CIWA, overview of alcohol use history, and confirmation of ability to connect daily via telemedicine (and one on-site visit) and the presence of social support during detox. Following risk/benefit discussion, AAW protocol was initiated after onset of alcohol withdrawal symptoms, or on the morning after last alcohol consumption. Biomarkers (EtG/ EtS) were obtained to confirm abstinence.
Results: AAW protocol included: Gabapentin $800 \mathrm{mg}$ PO/QID/five days; Valproic acid $500 \mathrm{mg} \mathrm{PO} / \mathrm{BID} / 15$ days; Clonidine $0.1 \mathrm{mg} \mathrm{PO}$ QID/PRN/five days; and Naltrexone $50 \mathrm{mg}$ PO daily, with planned transition to XR-IM naltrexone/month. Patient one: 64-year-old male with history of severe AUD and coronary artery disease (CAD) reported fogginess on day two, but otherwise did not experience breakthrough withdrawal, and later initiated XR-IM naltrexone. Patient two: 36-year-old male with history of moderate AUD felt tired by the third dose of gabapentin, decreased dose to $400 \mathrm{mg}$ TID, and further decreased to $400 \mathrm{mg}$ qHS on day four. He otherwise completed detoxification without incident. Patient three: 66-year-old male with history of severe AUD and CAD experienced minimal symptoms during detox and continued on gabapentin $400 \mathrm{mg}$ TID after day five. All remained on Valproic acid $\mathrm{BID} / 15$ days, naltrexone, and free of alcohol.

Conclusion: Patient selection and safe/effective protocol are key for telemedicine based AAW management. These cases highlight safe/effective use of a non-benzodiazepine-based protocol, utilizing primarily telemedicine contact during COVID-19 for AAW management.

095. Hypercobalaminemia Post Exogenous Administration Presenting with Paradoxical Acute Psychosis

Sheila Goertemoeller, Jonathan Colvin, Shan Yin

Cincinnati Children's Hospital Medical Center's Drug and Poison Information Center, Cincinnati, OH, USA

Background: Vitamin B12 or cobalamin is vital for cellular functioning. Although psychosis has been well described in the content of B12 deficiency, scant literature suggests that elevated levels can produce a similar clinical picture. Hypothesis: Hypercobalaminemia may present with paradoxical psychosis.

Methods: This is a single case report involving a 15-year-old previously healthy male of Guatemalan descent who presented with new onset auditory hallucinations and paranoia. He endorsed chest pain, headache, palpitations, dyspnea, dizziness, and tingling. His acute psychosis prompted a cobalamin level that exceeded measurable thresholds $>$ $20,000 \mathrm{pg} / \mathrm{mL}$ (normal 211-911 pg/mL). His father disclosed that he had been injecting his son with unclear doses of vitamin B12 for chest pain and tingling over a three-week period including the day of admission.

Results: The patient maintained unremarkable vitals, $\mathrm{CBC}, \mathrm{EKG}$ and head CT; however, his liver function tests were mildly elevated. The patient responded well to antipsychotics, and he was discharged home the following day. The father was instructed to refrain from further vitamin B12 injections. Vitamin B12 has a half-life of six days; therefore, the patient was instructed to report back in one week for a repeat level. The patient's seven-day outpatient level was markedly reduced $(7051 \mathrm{pg} / \mathrm{mL})$ and the patient denied any ongoing symptoms or hallucinations.

Discussion: Vitamin B12 is a water-soluble vitamin with an extraordinary safety margin. There are no specific antidotes and overdose generally requires discontinuation and follow up until symptoms have resolved. The exact reason for B12 administration in this patient remains unclear. It has been reported that the typical Guatemalan diet is deficient in this vitamin.

Conclusion: We report a case of paradoxical acute psychosis and elevated levels post exogenous administration of cobalamin.

096. Magnesium Toxicity after Oral Ingestion of a Colon Cleanse Powder

Antonia J Nemanich, Jenny Lu

Toxikon Consortium, Chicago, IL, USA

Background: Magnesium toxicity can cause severe symptoms and significant morbidity. Most cases of severe magnesium toxicity are iatrogenic and follow IV administration. Oral magnesium ingestion leading to significant toxicity is very rarely reported in the literature. Magnesium concentrations greater than $15 \mathrm{mg} / \mathrm{dL}$ are often fatal, though serum 
concentration does not always correlate well with the severity of symptoms. We present a case where the patient developed extremely elevated magnesium concentrations after taking a colon cleanse product by mouth yet had only moderate clinical symptoms.

Case: A 63-year-old woman with no significant past medical history took "a few tablespoons" of a colon cleanse preparation containing only powdered magnesium chloride which she mixed with water. The package contained $107 \mathrm{~g}$ of magnesium citrate in total. The patient had been fasting all day. Reportedly only five minutes after swallowing the product, she began to feel hot, dizzy, nauseous and generally weak. Vital signs were normal on arrival to the emergency department two hours later. Physical exam was significant for ataxia, with the patient too unsteady to walk, and hyporeflexia. She also developed severe diarrhea. The initial serum magnesium concentration was $15.0 \mathrm{mg} / \mathrm{dl}$ approximately three hours post-ingestion. Magnesium concentration peaked at $19.0 \mathrm{mg} / \mathrm{dl}$ one hour later. The patient had no renal insufficiency and serum creatinine was normal. She responded well to treatment with intravenous fluids, furosemide, and calcium gluconate. She never developed any hypotension, arrhythmias, respiratory depression, or severe central nervous system depression. She was admitted to the ICU and discharged asymptomatic three days later.

Conclusion: Colon cleansing preparations can be a rare cause of significant magnesium toxicity. While severe symptoms have been reported following ingestion of epsom salts, a concentration of $19 \mathrm{mg} / \mathrm{dl}$ is significantly higher than any previously reported from oral exposure to magnesium.

\section{Liver Injury Due to a Performance-Enhancing Supplement}

Muhammad Akbar Baig, Joshua Nogar

North Shore University Hospital, Manhasset, NY, USA

Background: Drug induced liver injury (DILI) is an important cause of iatrogenic liver injury and often goes unrecognized and underreported. Performance-enhancing nutritional supplements (PENS) are a popular commodity used during workouts for promoting muscle gain and improving exercise performance.

Method: This is a case report of a 45-year-old man who presented to the ED with painless jaundice. He had no related occupational exposures, was not taking prescription medications, and did not use recreational drugs or alcohol. Recently, he modified his lifestyle with exercise, and was using creatine and whey-based protein supplements for the past two weeks prior to the development of symptoms. Upon presentation, laboratory tests revealed elevated total bilirubin $(3.7 \mathrm{mg} / \mathrm{dL})$, transaminases (AST/ALT 640/2478 U/L) and alkaline phosphatase (230 U/L). Serologic workup was negative for viral hepatitis and autoimmune liver disease. Magnetic resonance cholangiopancreatogram was unremarkable. He had dramatic clinical improvement after discontinuation of the nutritional supplement and was discharged with a diagnosis of DILI due to consumption of a PENS.

Results: Most cases of DILI are idiosyncratic and while it is clear that pharmacogenomics plays some kind of role in pathogenesis of DILI, some cases may be caused by the unwitting consumption of innate hepatotoxins. Seeing as PENS are not stringently regulated in comparison to FDA-approved xenobiotics, confusion will remain the norm as to the exact etiology of PENS-associated DILI, because: 1 ) there is heterogeneity between products; 2) Variety of products are used simultaneously; 3) products are collectively discontinued upon hospital admission, rather than sequentially removed; 4) there is a lack of clinical analytical tools to assay products; and 5) the product may be unavailable for clinicians to analyze.

Conclusion: DILI secondary to PENS, will continue to puzzle clinicians, hence we should employ a multidisciplinary approach for evaluating such cases.
098. Something to Gripe About: A Gripe Water Aspiration Event Resulting in Hemoptysis, Pneumomediastinum, and Aspiration Pneumonia

Svetlana Duvidovich, John Park

Maria Fareri Children's Hospital - Westchester Medical Center Health Network, Valhalla, NY, USA

Background: Gripe water is a heterogeneous, comparatively unregulated remedy composed of ingredients such as herbal oils and is often used for infantile colic. Some formulations contain undissolved ingredients which may be difficult to swallow and present a choking hazard. Since gripe water is often marketed as an herbal supplement, it can bypass U.S. Food and Drug Administration approval.

Hypothesis: Gripe water may have adverse effects related to choking including hemoptysis, pneumomediastinum, and aspiration pneumonia. Methods: This is a single patient chart review. A one-month-old previously healthy female presents with hemoptysis and dark stools following a choking episode on gripe water given for colic. During administration via oral syringe, the infant had a severe episode of coughing associated with cyanosis. The episode was self-resolved. Shortly after, the infant developed blood-tinged secretions and dark stools.

Results: On evaluation, the patient was well appearing but based on history was worked up. Labs without anemia or electrolyte derangement. Stool with occult blood. Symptoms presumed to be GI related, so an abdominal $\mathrm{x}$-ray was obtained. Captured lung fields showed consolidation and pneumomediastinum. Chest X-ray (CXR) showed left airspace opacity with pneumomediastinum tracking up the neck. Patient developed tachypnea and distress so started on oxygen via nasal cannula. Admitted to Pediatric Intensive Care Unit for pneumomediastinum and concern for pulmonary hemorrhage. Treated with Methylprednisolone, Protonix, and Augmentin for presumed aspiration given left airspace opacity. Serial CXR showed improvement of left sided opacity and pneumomediastinum. Patient was successfully weaned to room air and remained hemodynamically stable so CT Angiogram and Bronchoscopy deferred.

Conclusion: Ingesting gripe water may lead to morbidity as in this case with ICU admission secondary to choking events possibly tied to undissolved solids; this is a relatively unrecognized hazard with gripe water well recognized to have toxic effects when formulated with other active ingredients.

\section{Review of Recent Herbicide Exposures}

Olivia Burris ${ }^{1}$, Edric Wong ${ }^{1}$, Nicholas Dodson ${ }^{1}$, Tony Rianprakaisang ${ }^{2}$, On Behalf of the Toxicology Investigators Consortium (ToxIC)

${ }^{1}$ The University of Kansas Health System, Kansas City, KS, USA. ${ }^{2}$ University of Kansas Medical Center, Kansas City, KS, USA

Background: Herbicides are a relatively uncommon exposure and encompass a breadth of agents with varying toxicities.

Research Question: What are the agents of exposure, demographics, and clinical characteristics of patients exposed to herbicides?

Methods: This is a retrospective review of all single-substance herbicide exposures reported to the Toxicology Investigators Consortium Registry from January 2010 through May 2021.

Results: Fifty-seven single-substance herbicide exposures were identified. Of these, $71.9 \%$ were male. Exposures were intentional in $22.8 \%$ of cases. The most common agents identified were glyphosate $(33.3 \%)$, Agent Orange/chlorphenoxy herbicides (19.3\%), paraquat (12.3\%), and dicamba (8.8\%). Inhalational (33.3\%), oral $(29.8 \%)$, and dermal $(12.2 \%)$ routes of exposure were most common. The nature of the exposure was most often acute $(57.9 \%$ ), with $22.8 \%$ being chronic and $19.3 \%$ unknown/not reported. More than half of the encounters occurred in the outpatient setting (50.8\%). Clinical outcomes were generally good, with death occurring in only three cases $(5.2 \%)$; however, all three cases were intentional self-harm exposures to paraquat. Nine patients (15\%) had a major vital sign abnormality reported, which included hypotension $(\mathrm{n}=$ 
5), tachycardia $(\mathrm{n}=2)$, hypertension $(\mathrm{n}=1)$, and bradycardia $(\mathrm{n}=1)$. The most common pharmacologic support provided was vasopressors, steroids, and benzodiazepines, each of which was utilized in four patients (7.0\%). Among non-pharmacologic support, $14.0 \%$ received IV fluid resuscitation and $8.8 \%$ were intubated or required ventilatory management. Activated charcoal was used in three patients $(5.3 \%)$. Extracorporeal removal via hemodialysis or continuous renal replacement was used in four patients (7.0\%). N-acetylcysteine was administered in two paraquat exposures, both of which were fatal.

Conclusion: Single-agent herbicide exposures were relatively welltolerated with the exception of paraquat, which was responsible for all deaths from single-agent herbicide exposure. More research may be warranted to further characterize herbicide exposures and outcomes.

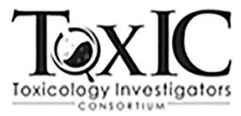

This research was perforn

\section{Review of Recent Rodenticide Exposures}

Olivia Burris $^{1}$, Edric Wong ${ }^{1}$, Nicholas Dodson ${ }^{1}$, Tony Rianprakaisang ${ }^{2}$, On Behalf of the Toxicology Investigators Consortium (ToxIC)

${ }^{1}$ The University of Kansas Health System, Kansas City, KS, USA.

${ }^{2}$ University of Kansas Medical Center, Kansas City, KS, USA

Background: Rodenticides are a relatively uncommon exposure and encompass a wide variety of agents with varying toxicities.

Research Question: What are the agents of exposure, demographics, and clinical characteristics of patients exposed to rodenticides?

Methods: This is a retrospective review of all single-substance rodenticide exposures reported to the Toxicology Investigators Consortium Registry from January 2010 through May 2021.

Results: One hundred and four single-substance rodenticide exposures were identified. Of those, $65.4 \%$ were male. Exposures were intentional in $51.9 \%$ of cases. The most common agents identified were brodifacoum $(52.9 \%)$ followed by bromethalin $(10.6 \%)$, and $68.2 \%$ of cases involved exposure to a vitamin $\mathrm{K}$ antagonist. The most common route of exposure was oral $(65.4 \%)$, and exposures were most often acute $(73.1 \%)$. There were no reported deaths, and only two patients $(1.9 \%)$ had a major vital sign abnormality, which included one case with hypotension (SBP < $80 \mathrm{~mm} \mathrm{Hg}$ ) and one with tachycardia (Pulse > 140). Vitamin K was the most common treatment given $(23.1 \%)$; however, only one patient required anticoagulant reversal with factor repletion. Among nonpharmacologic support, six patients $(5.8 \%)$ received IV fluid resuscitation, four $(3.8 \%)$ were intubated or required ventilatory management, three $(2.9 \%)$ received a transfusion, and one $(1.0 \%)$ underwent therapeutic hypothermia. Decontamination with activated charcoal was used in three patients $(5.3 \%)$, and no patient required any enhanced elimination therapies.

Conclusion: Vitamin $\mathrm{K}$ antagonists are the most common rodenticide exposure type. While treatment may be necessary, single-agent exposures appear to have good clinical outcomes with low morbidity and mortality.

\section{Toxicology Invostigators}

101. No Fly Zone: An Unregistered Insecticide Containing Dichlorvos Resulting in Cholinergic Toxicity

Nicholas P Johnson, Derek J Fikse, Ryan M Surmaitis, Alexandra M Amaducci

Lehigh Valley Health Network, Department of Emergency and Hospital Medicine/USF Morsani College of Medicine, Allentown, PA, USA
Background: 2,2-dichlorovinyl dimethyl phosphate (DDVP, Dichlorvos) is an organophosphate insecticide causing cholinergic toxicity through acetylcholinesterase inhibition. Its use is restricted in the United States (US) and reports of toxicity are rare.

Case Report: A 70-year-old male presented to the Emergency Department with vomiting and unresponsiveness. Initial vital signs were: BP 91/57 mmHg, HR 32 bpm, temperature $95.8^{\circ} \mathrm{F}, \mathrm{RR} 20, \mathrm{SpO} 295 \%$. The patient was obtunded with excessive airway secretions and required emergent intubation. His physical examination demonstrated increased lower extremity muscle tone and muscle fasciculations. Atropine (1 $\mathrm{mg}$ ) was administered with immediate resolution of excess secretions and bradycardia. It was reported the patient accidently ingested dichlorvos which was stored next to his mouthwash. He received pralidoxime chloride $2 \mathrm{~g}$, midazolam $5 \mathrm{mg}$, and norepinephrine infusion. An additional $2 \mathrm{mg}$ of atropine was needed for recurrent bradycardia. Abnormal labs included: bicarbonate $20 \mathrm{mmol} / \mathrm{L}$ [23-31], anion gap 14 [3-11], lactate 4.6 $\mathrm{mmol} / \mathrm{L}$ [0.5-2.1], and $\mathrm{pH} 7.30$ [7.35-7.45]. He was admitted to the ICU and extubated on hospital day 2. RBC cholinesterase level was $23.8 \mathrm{U} / \mathrm{g}$ $\mathrm{Hb}$ [31.2-61.3]. The patient did not develop intermediate syndrome or organophosphate-induced delayed neurotoxicity and had a full recovery. Discussion: Dichlorvos is a common organophosphate in developing nations, but its use is restricted in the US. There have been federal advisory alerts warning of unregistered dichlorvos products, specifically Sniper DDVP $\odot$ and NoPest $\odot$, being illegally transported/sold in the US. Our patient obtained dichlorvos from the Dominican Republic to use as a household insecticide. Accidental ingestions may have significant morbidity and mortality. Similar to most organophosphate poisonings, treatment includes decontamination, atropine, pralidoxime, and benzodiazepines.

Conclusion: Dichlorvos poisoning is rare in the US but remains potentially lethal, requiring early recognition of the cholinergic toxidrome and immediate treatment. Safe storage of harmful chemicals, away from pharmaceutical agents, is imperative.

102. Clinical Effects from Ingestion of Multiple Plant Products Purchased via the Internet: Aconitum Alkaloid Poisoning with Confirmatory Testing

Daniel J McCabe ${ }^{1,2}$, Kara L Lynch ${ }^{3,4}$, Joshua B Radke ${ }^{1,2}$, Bryan Z Wilson ${ }^{1,2}$

${ }^{1}$ University of Iowa, Iowa City, IA, USA. ${ }^{2}$ Iowa Poison Control Center, Sioux City, IA, USA. ${ }^{3}$ Zuckerberg San Francisco General Hospital, San Francisco, CA, USA. ${ }^{4}$ University of California-San Francisco, San Francisco, CA, USA

Background: With rare confirmation of products provided, the internet has allowed access to unregulated "natural" products producing an unlimited number of indications for use and subsequent physical effects. Aconitum alkaloids are present in numerous natural sources and increase membrane permeability to sodium ions which can produce lifethreatening neurologic and cardiac effects.

Methods: (First Presentation) An adult male with daily kratom use presented after intentional ingestion of an internet-purchased product marketed as wolfsbane root. He boiled and ingested 2 grams of the substance about one hour prior to arrival. He presented with blurry vision, parasthesias, hot-cold sensation reversal, and myokymia in multiple muscle groups. Serial EKGs revealed a new wide complex tachycardia unresponsive to sodium bicarbonate treatment and progressive QT interval prolongation. (Second Presentation) The same patient presented one month later after an intentional ingestion of multiple herbal products. He purchased seeds, powders, and plant material from multiple internet websites to produce an experience similar to ayahuasca. He parboiled and drank a liter consisting of belladonna, mandrake, mimosa hostilis root bark, syrian rue, hawaiian woodrose seed and voacanga root bark. $\mathrm{He}$ was asymptomatic with a normal exam and EKG.

Results: Urine from both presentations was analyzed by liquid chromatography-high-resolution mass spectrometry for toxic natural 
products. First Presentation: lappacotinine (Aconitum sinomontanum), mitragynine, 7-hydroxymytragynine, trigonelline. Second Presentation: bufotenine, dimethyltryptamine, harmaline, harmine, psilocin, trigonelline. Present in both samples: risperidone, 9-hydroxyrisperidone, hydroxyzine, cotinine, nicotine.

Conclusion: The ability to buy products from anywhere in the world for unsubstantiated indications can have devastating consequences. Despite Aconitum sinomontanum's reported uses in Eastern medicine and sporadic cases of death from Aconitum alkaloids, it is exceedingly rare for a case of poisoning to be confirmed. We present a case of intentional ingestion of multiple unregulated products purchased via the internet with corresponding clinical effects and confirmatory testing of exposure.

\section{Cardiomyopathy and Hepatotoxicity Related to Kratom Use} Arthur W Daigh, Aimee Kabala, Laura M Tormoehlen IU Health, Indianapolis, IN, USA

Background: Kratom is a drug of abuse with mixed stimulant and opioid agonist properties and has been used as an herbal treatment for opioid withdrawal. There are rare case reports of kratom causing cardiomyopathy and hepatotoxicity.

Methods: This is a single patient case review. A 29-year-old man without known cardiac dysfunction presented to the hospital in cardiogenic shock and was found to have new onset heart failure and hepatitis. At age ten, he was treated for non-Hodgkin's lymphoma with a regimen including doxorubicin. He developed opioid use disorder but switched to Kratom three years ago. Two weeks prior to presentation, he switched products and increased his intake. He presented with three days of shortness of breath and abdominal fullness. Initial vitals were: HR 130 beats/min, BP 101/78 $\mathrm{mmHg}, \mathrm{T} 98.2^{\circ} \mathrm{F}, \mathrm{RR} 18$ breaths/min, $\mathrm{SpO} 2100 \%$. His exam was notable for respiratory distress with clear lungs. He underwent extensive evaluation to exclude other common causes of cardiomyopathy and hepatotoxicity.

Results: Initial laboratory evaluation showed unremarkable CBC, urinalysis, and blood cultures. Transaminases peaked at $5588 \mathrm{U} / \mathrm{L}$ and $3645 \mathrm{U} / \mathrm{L}$ for AST and ALT respectively on hospital day four. Viral hepatitis panel, acetaminophen, salicylate, and alcohol levels were negative. Right upper quadrant ultrasound was unremarkable. Troponin was elevated at $37 \mathrm{ng} / \mathrm{L}$ and EKG was without ischemic changes. TTE showed dilated LV with EF $<10 \%$ and LV thrombus. Patient was started on heparin and furosemide for diuresis. He also required norepinephrine and dobutamine. He was weaned off vasopressors hospital day seven. Cardiac MRI performed hospital day nine showed dilated cardiomyopathy with LVEF $12 \%$ without enhancement, and the patient was discharged that day. His symptoms improved at one-week follow up. Results of a follow-up echocardiogram are pending. Conclusion: We suspect that the patient's cardiomyopathy and hepatitis may have been due to kratom toxicity although other causes cannot completely be ruled out.

104. Syncope and Prolonged QT Interval Associated with Chronic Ingestion of Mitragyna Speciosa (Kratom) Jesse S Smith ${ }^{1}$, Rehan M Karim ${ }^{2}$, Stephen W Smith ${ }^{1,2}$, Travis D Olives ${ }^{2,3}$ ${ }^{1}$ University of Minnesota Medical School, Minneapolis, MN, USA. ${ }^{2}$ Hennepin County Medical Center, Minneapolis, MN, USA. ${ }^{3}$ Minnesota Poison Control System, Minneapolis, MN, USA

Background: Mitragyna speciosa (kratom) is a medicinal plant commonly employed for anxiety, pain, depression, and as an opioid substitute. Kratom's active components have an inhibitory effect on cardiomyocyte rectifier potassium channels (IKr) via hERGla inhibition. Though kratom is not regulated under the Controlled Substances Act, reports of its use are increasing. Methods: Single patient report of a previously healthy 22-year-old female presenting to a busy urban Emergency Department (ED) after a firsttime seizure.

Results: The patient presented following a witnessed sudden loss of consciousness, with one to two minutes of tonic-clonic movements. She presented tachycardic and tachypneic but otherwise stable. Head CT and basic labs were reassuring; mild hypokalemia was noted. Urine drug immunoassay was negative, with confirmation pending. Twelve-lead EKG revealed an erroneous computerized QT of $422 \mathrm{~ms}$ (QTc $485 \mathrm{~ms}$ by Hodges correction). In consultation, neurology deferred anti-epileptic medications. Subsequent manual overread of the EKG, correcting prolonged QRS, revealed a QT of $470 \mathrm{~ms}$, and QTc of $552 \mathrm{~ms}$ (Hodges formula) and $628 \mathrm{~ms}$ (Bazett). She was recalled to the ED, where she reported regular kratom use (last use ten hours before initial presentation). She was admitted for electrophysiology consultation regarding possible long QT syndrome (LQTS). Confirmatory urine testing returned only mitragynine and ibuprofen. An implantable defibrillator was deferred in favor of Holter monitoring given the presence of mitragynine. On follow-up she reported reduced but consistent kratom use. Holter revealed a QTc $>460 \mathrm{~ms}$ for $75.4 \%$ of the monitoring period (longest recorded QTc $519 \mathrm{~ms}$ ).

Conclusion: Drug-induced $\mathrm{IKr}$ channel inhibition is linked to long QT syndrome. Kratom ingestion may pose a risk for inducing novel long QT or exacerbating existing LQTS. In this case, kratom likely contributed to her syncope leading to a presumed diagnosis of LQTS. Kratom use should be considered in new cases of LQTS and cardiogenic syncope in healthy individuals.

105. The New Trend: Delta-8 THC Exposure Confirmed in Four Pediatric Cases

Kerollos A Shaker ${ }^{1,2}$, Andrea Nillas ${ }^{1,2}$, Roy R Gerona ${ }^{3}$, Ross Ellison ${ }^{3}$, Kim Aldy ${ }^{1,4}$

${ }^{1}$ University of Texas Southwestern Medical Center, Dallas, TX. ${ }^{2}$ Parkland Health \& Hospital System, Dallas, TX. ${ }^{3}$ University of California at San Francisco, San Francisco, CA. ${ }^{4}$ American College of Medical Toxicology, Phoenix, AZ

Background: Delta-8 tetrahydrocannabinol (THC) is an isomer of delta9-THC, and both are found naturally in the Cannabis sativa plant. Delta8-THC has similar potency to Delta-9-THC. Delta-8-THC is not yet regulated in many states and widely accessible in retail shops, leading to a rise in pediatric exposures to delta-8-THC products with consequential clinical effects.

Research Question: What are the clinical effects of delta-8-THC in pediatric exposure?

Methods: This is a case series of four pediatric patients seen between June 2021 to September 2021 at one urban children's hospital with confirmed exposure to delta-8-THC. Urine samples were submitted for a basic immunoassay drug screen. Subsequent confirmatory drug analysis of residual biological samples of blood/urine via LC-Quadrupole Timeof-Flight Mass Spectrometry was performed with the assistance of the Drug Enforcement Administration's Toxicology Testing Program and the UCSF Clinical Toxicology and Environmental Biomonitoring Lab.

Results: Three patients had presumed ingestion of edibles, one of which was known ingestion to delta-8-THC gummy bear product. The fourth patient vaped delta-8-THC at school. Ages ranged from 18-months to 16years-old, with an average age of seven years old. Clinical effects ranged from confusion, somnolence, seizure-like activity, hypotension, and tachycardia. All four patients tested positive for cannabinoids on the urine drug screen immunoassay. Confirmatory drug analysis for all four patients detected 11-nor-9-carboxy-delta-8-THC, which is a metabolite of delta-8-THC. No delta-9-THC was found. Quantified levels in the samples are $31.6-929 \mathrm{ng} / \mathrm{mL}$ for serum/plasma and 109-292 ng/mL for urine. Conclusion: It is imperative to realize that there is an increased incidence of pediatric patients being exposed to delta-8-THC given the increased availability of delta- 8 -THC products. In the pediatric population, the clinical effects of central nervous system depression, seizures, hypotension, and tachycardia of delta-9-THC can also be seen with delta-8-THC.

106. A Chocolate Shake: Acute THC-infused Edible Ingestion with Sustained Inducible Clonus

Keahi M Horowitz, Robert Schwaner, Jeremy Hardin, Ryan Earwood, Seth Burack, Jose Torres, James Tavornwattana 
Stony Brook University Hospital, Dept. of Emergency Medicine, Stony Brook, NY, USA

Background: Tetrahydrocannabinol (THC)-infused food products ("edibles") are becoming more readily available after several state laws legalized their production and sale. However, most state regulations do not limit how much THC any single package can contain. As demonstrated here, an acute ingestion of a single package of edibles can cause toxicity with serotonergic features, particularly sustained inducible clonus and hyperreflexia.

Methods: In this single patient case, a college-aged male presented obtunded after ingesting one small, 22-gram box with nine pieces of THC-infused chocolates. Initial vital signs: heart rate 102 beats-perminute, oral temperature 37 degrees celsius, and blood pressure 162/72 $\mathrm{mmHg}$. Due to copious emesis, emergent intubation was considered, but the patient improved, demonstrating unlabored respirations, oxygen saturations $>96 \%$ on room air, and no further vomiting. Physical examination revealed hyperreflexia and inducible, sustained clonus of the lower extremities but not of the upper extremities. Supportive care was provided until further history could be obtained.

Results: Diagnostic workup revealed a urine drug screen positive for cannabinoids only. After eighteen hours of observation, the patient became more alert. He confirmed consumption of only the nine edible pieces - a total of $216 \mathrm{mg}$ of THC ( $225 \mathrm{mg}$ total cannabinoids) according to the product label - but denied knowledge that the chocolates contained THC, suggesting incidental ingestion. He denied any other drug or alcohol use, specifically no serotonin-affecting medications. During observation, his lower extremity clonus persisted even as his mental status improved but resolved by discharge on hospital day two

Conclusion: Acute, high-dose exposures to THC products can cause toxicity with features of serotonin syndrome. Although previously reported in highly concentrated inhalation exposures of butane hash oil, this case report demonstrates that even single packages of edibles can contain toxic concentrations of THC.

107. Cardiovascular Complications of Massive Tetrahydrocannabinol Ingestion in a Pediatric Patient

Marielle A Brenner ${ }^{1,2}$, Blake A Froberg ${ }^{1,2}$

${ }^{1}$ Indiana University, Indianapolis, IN, USA. ${ }^{2}$ Indiana Poison Center, Indianapolis, IN, USA

Background: Use of concentrated tetrahydrocannabinol (THC) products by pediatric patients are resulting in previously rare complications of THC use including cardiovascular complications. Study Aim: To describe a case of cardiovascular complications after high dose THC use in a pediatric patient.

Methods: This is a single patient chart review. A sixteen-year-old female presented with central nervous system depression, hypotension, and bradycardia after an intentional recreational ingestion of $1.2 \mathrm{~g}$ THC store bought gummy candies. Presenting vital signs included a blood pressure $95 / 56 \mathrm{mmHg}$, heart rate 65 beats per minute. She awoke to sternal rub on initial exam and had a Glascow Coma Score of 10 (motor $=$ five, verbal $=$ three, eye $=$ two) without other focal findings. Laboratory findings were remarkable only for cannabinoids on urine drug screen and head CT was negative.

Results: Her heart rate had a nadir of 47 beats per minute and her blood pressure had a nadir of $84 / 57 \mathrm{mmHg} 24$ hours after reported initial ingestion. Her hypotension was treated with a total of six liters of intravenous fluid prior to initiation of a norepinephrine infusion. After five hours, norepinephrine was weaned. Four hours after the norepinephrine was weaned, she had several episodes of bigeminy lasting approximately two to five minutes each. Her potassium and magnesium were within normal limits during her hospitalization. By hospital day four, her mental status had improved, her heart rate and blood pressure had normalized, and her bigeminy had resolved, allowing for hospital discharge. Careful history and medication reconciliation by the medical toxicology team revealed no other probable cause for her symptoms.
Conclusions: Ingestions of high doses of THC can lead to bradycardia, hypotension, and bigeminy arrhythmia in pediatric patients.

108. Urine Fentanyl and Norfentanyl Concentrations in Emergency Department (ED) Patients With Opioid Related Visits

Austin Kilaru $^{l}$, Sophia Faude ${ }^{l}$, Mucio Kit Delgado ${ }^{l}$, Anthony Spadaro ${ }^{l}$, Ashish Thakrar ${ }^{1}$, Lewis S Nelson ${ }^{2}$, Margaret Lowenstein ${ }^{1}$, Jeanmarie Perrone $^{1}$

${ }^{I}$ Perelman School of Medicine, University of Pennsylvania, Philadelphia, PA, USA. ${ }^{2}$ Rutgers New Jersey Medical School, Newark, NJ, USA

Background: Fentanyl prevalence and detection in the illicit drug supply and in patients with opioid use disorder has escalated. There is no data on how urine fentanyl and norfentanyl concentrations relate to clinical effects.

Research Question: Do urine fentanyl and norfentanyl concentrations in ED patients differ between patients presenting for opioid overdose versus withdrawal?

Methods: We conducted a retrospective study of opioid-related ED visits from January 2020-March 2021 in three Philadelphia EDs. We included patients with both ICD-10 codes for opioid-related visits and a urine drug test (UDT). We identified patient demographics, clinical presentation (opioid overdose, withdrawal, other), and visit date. We classified patients into low $(<400 \mathrm{ng} / \mathrm{mL})$ or high $(>400 \mathrm{ng} / \mathrm{mL})$ urine fentanyl and low $(<1000 \mathrm{ng} / \mathrm{mL})$ or high $(>1000 \mathrm{ng} / \mathrm{mL})$ urine norfentanyl concentrations. We used multivariable logistic regression to determine the association between clinical presentation and patient characteristics, including a time trend by quarter. We report descriptive statistics and adjusted odds ratios by clinical presentation and quarter.

Results: Four thousand and seventy-five patients had a UDT collected (63.7\% male, mean age 43.7), and 1224 identified fentanyl (30\%). The proportion with high urine fentanyl concentrations increased per quarter (OR 1.10, 95\% CI 0.99-1.21). Withdrawal patients were significantly more likely to have high fentanyl concentrations (OR 1.44; $95 \%$ CI 1.01-2.07) than overdose patients. Urine norfentanyl was detected in 1253 of 4075 patients $(30.7 \%)$. High norfentanyl concentrations significantly increased per quarter (OR $1.13 ; 95 \%$ CI 1.04-1.23). Withdrawal patients were significantly more likely to have high norfentanyl concentrations (OR 1.71, 95\% CI 1.20-2.43) compared to patients with overdose.

Conclusion: Among patients with opioid-related ED visits and UDT, the proportion of patients with high urine fentanyl and norfentanyl concentrations increased over time. Patients with opioid withdrawal had a greater likelihood of having high fentanyl and norfentanyl concentrations. Further evaluation of fentanyl and norfentanyl concentration relationships may help interpret these findings.

109. Para-Fluorofentanyl Detected in Patients with Suspected Opioid Overdose

Kim Aldy ${ }^{1,2}$, Alex Krotulski ${ }^{3}$, Sharan Campleman ${ }^{1}$, Alison Meyn ${ }^{1}$, Stephanie Abston ${ }^{1}$, Barry Logan ${ }^{3,4}$, Jeffrey Brent ${ }^{5}$, Paul Wax ${ }^{1}$, Alex F Manini ${ }^{6}$, On Behalf of the Toxicology Investigators Consortium (ToxIC) Fentalog Study Group

${ }^{1}$ American College of Medical Toxicology, Phoenix, AZ, USA.

${ }^{2}$ University of Texas Southwestern Medical Center, Dallas, TX, USA.

${ }^{3}$ Center for Forensic Science Research and Education at the Fredric Rieders Family Foundation, Willow Grove, PA, USA. ${ }^{4}$ NMS Labs, Horsham, PA, USA. ${ }^{5}$ University of Colorado School of Medicine, Aurora, CO, USA. 'Icahn School of Medicine at Mount Sinai, New York, NY, USA

Background: Para-fluorofentanyl (PFF), a fentanyl analog, is a schedule one drug with no accepted medical use in the US. PFF is commonly found in combination with illicit fentanyl, but occasionally alone, and may reflect a change in the use of precursors in illicit fentanyl manufacture. No reports of its clinical effects exist in the literature to date. 
Research Question: This study aims to investigate PFF exposures. Methods: This is a prospective cohort of adult emergency department patients with presumed opioid overdose presenting to nine US sites with available waste blood. Exclusion criteria were non-toxicologic diagnosis, pediatrics, and prisoners. Residual blood testing via liquid chromatography quadrupole time-of-flight mass spectrometry for over 900 substances was paired with clinical data for analysis.

Results: Between 10/6/20-10/31/21, 59 of 412 patients (14.3\%) were PFF positive. Average age was 42 years; $61 \%$ were male $(n=36)$. Heroin was the most common self-reported exposure $(n=29,49.2 \%)$, but only 13 had heroin on confirmatory testing. Fentanyl exposure was reported by nine (15.3\%), but $97 \%$ had fentanyl present. Forty-seven $(80 \%)$ received at least one naloxone dose. The most common indications were depressed level of consciousness $(n=34,72.3 \%)$ and respiratory depression $(\mathrm{n}=30,63.8 \%)$. After initial naloxone, 10 (21.3\%) had no response, necessitating additional doses. Cardiac arrest prior to naloxone occurred in one that ultimately succumbed to brain death. Quantification ratios of fentanyl to PFF by comparison of peak area showed six had more PFF than fentanyl. In one case, the PFF amount was 38 times greater than fentanyl.

Conclusion: PFF was present in $14.3 \%$ of our presumed opioid overdose cohort, with fentanyl also in $97 \%$. Decreased consciousness and respiratory depression were most common, and nearly $1 / 5$ had no initial naloxone response. Though low potency has been described, these findings suggest it may have more serious clinical consequences than previously thought.

\section{ToxIC}

This research was performed in collaboration with the ACMT Toxicology Investigators Consortium.

110. Micro-dosing Buprenorphine In A Patient Chronically Receiving High-Dose Fentanyl and Morphine

Raleigh Ems ${ }^{1}$, Lauren Porter ${ }^{2}$, Ryan Marino ${ }^{2}$

${ }^{1}$ Department of Emergency Medicine, University Hospitals Cleveland Medical Center \& Case Western Reserve University School of Medicine, Cleveland, OH, USA. ${ }^{2}$ Division of Medical Toxicology \& Addiction Medicine, Department of Emergency Medicine, University Hospitals Cleveland Medical Center \& Case Western Reserve University School of Medicine, Cleveland, OH, USA

Background: Buprenorphine is a partial agonist of the mu-opioid receptor and binds with higher affinity than typical opioids. Because of these pharmacologic properties, initiation of buprenorphine in a patient chronically taking high doses of opioids can precipitate severe withdrawal.

Hypothesis: Micro-dosing buprenorphine can safely bridge patients on high doses of chronic opioids, including fentanyl, without the need for a 'washout' period that requires patients to undergo opioid withdrawal and without precipitating iatrogenic opioid withdrawal.

Methods: This is a single patient case report. A 34-year-old man with a history of opioid use disorder presented to a tertiary academic center with endocarditis complicated by septic pulmonary emboli and septic arthritis. Because of the severity of his illness, he was initially made palliative with patient-controlled analgesia (PCA) pump containing fentanyl in addition to scheduled oral morphine. The patient responded well to medical management and underwent multiple surgical procedures to control the sources of his infections. Post-operatively, the patient requested assistance discontinuing opioids and a desire for buprenorphine therapy.

Results: On his $35^{\text {th }}$ day on fentanyl PCA and scheduled morphine, the patient was started on a dose of $0.075 \mathrm{mg}$ of intravenous buprenorphine every 12 hours while still receiving $15 \mathrm{mcg}$ of fentanyl every 15 minutes and $22.5 \mathrm{mg}$ oral morphine every two hours. The dosing of intravenous buprenorphine was slowly increased over 16 days to four mg every eight hours with minimal to no adverse effects while simultaneously reducing the frequency and quantity of dosing from both the fentanyl PCA and the oral morphine. The patient was then transitioned to sublingual buprenorphine, which was increased up to eight mg every eight hours and eventually discharged on hospital day 87 on buprenorphine alone.

Conclusion: Micro-dosing induction with buprenorphine can allow patients on high doses of chronic opioids, including fentanyl, to transition to buprenorphine without undergoing opioid withdrawal.

111. Micro-dosing Buprenorphine in Patients on Full-dose Opioids: A Case Series

Lauren M Porter ${ }^{1,2}$, Ryan Marino ${ }^{1,2}$, Bryan Ross ${ }^{1,2}$

${ }^{1}$ Division of Medical Toxicology and Addiction Medicine, University Hospitals Cleveland Medical Center, Cleveland, OH, USA.

${ }^{2}$ Department of Emergency Medicine, Case Western Reserve University School of Medicine, Cleveland, OH, USA

Background: Buprenorphine is a first line treatment for opioid use disorder (OUD), however, as a partial mu-opioid receptor agonist with high binding affinity it requires most patients to either experience moderate withdrawal or abstain from opioids prior to induction. Micro-dosing buprenorphine can prevent the need for withdrawal prior to starting treatment. Hypothesis: IV or oral buprenorphine micro-dosing can safely bridge patients on other opioids while avoiding opioid withdrawal.

Methods: This is a case series of three patients induced on buprenorphine while on full opioid agonist therapy. Case one: 53-year-old female with a history of OUD transferred for operative management of a spinal abscess. She was started on $0.15 \mathrm{mg}$ IV buprenorphine on HD two while continuing morphine. She was transitioned to sublingual buprenorphine on HD four and reached therapeutic dosing on HD five. PO morphine was added on HD eight and the patient was discharged to rehab on HD13 on buprenorphine and morphine PRN. Case two: 56-year-old male with a history including OUD was with rib fractures and pneumothoraxes. He had been started on a hydromorphone PCA at $0.3 \mathrm{mg}$ Q six minutes and $0.8 \mathrm{mg}$ PRN Q1hr. He was started on IV buprenorphine on HD five and was weaned off his PCA on HD six. He was transitioned to sublingual buprenorphine on HD seven and was able to be discharged on his home dose of $12 \mathrm{mg}$ SL on HD 8. Case three: 48-year-old female transferred due to limb ischemia secondary to a cardiac arrest. She has a history including OUD with heroin and fentanyl. On HD nine she was started on IV buprenorphine and tolerated a transition to sublingual dosing on HD 13. Her remaining IV opioids were weaned by HD 20 while undergoing operative management of her limb ischemia.

Results: All patients were successfully induced with buprenorphine without withdrawal symptoms.

Conclusion: Micro-dosing of buprenorphine should be considered for patients on full agonist therapy.

112. Psychostimulant Drug Co-ingestion in Emergency Department Patients with Opioid Overdose: a Multi-Center ToxIC Collaboration Siri Shastry ${ }^{1}$, Kim Aldy ${ }^{2,3}$, Sharan Campleman ${ }^{3}$, Alison Meyn ${ }^{3}$, Stephanie Abston ${ }^{3}$, Alex Krotulski ${ }^{4}$, Barry Logan ${ }^{4,5}$, Jeffrey Brent ${ }^{6}$, Paul Wax ${ }^{2,3}$, Alex F. Manini ${ }^{1}$, On Behalf of the Toxicology Investigators Consortium (ToxIC)

${ }^{I}$ Icahn School of Medicine at Mount Sinai, New York, NY, USA. ${ }^{2}$ University of Texas Southwestern Medical Center, Dallas, TX, USA. ${ }^{3}$ American College of Medical Toxicology, Phoenix, AZ, USA. ${ }^{4}$ Center for Forensic Science Research and Education at the Fredric Rieders Family Foundation, Willow Grove, PA, USA. ${ }^{5}$ NMS Labs, Horsham, PA, USA. ${ }^{6}$ University of Colorado School of Medicine, Aurora, CO, USA

Background: In 2017, psychostimulant deaths involving cocaine and methamphetamine both increased by $>30 \%$. Opioid co-exposure with psychostimulants is a major driver of this increase in overdose deaths. Characterization of clinical outcomes in patients with exposure to fentanyl/fentanyl analogs (fentalogs) and psychostimulants is critical but few studies have examined outcomes in this population. 
Research Question: This study aims to investigate outcomes in patients with exposure to fentanyl and psychostimulants and compare outcomes in patients with fentanyl/psychostimulants to those with fentanyl/fentalogs only.

Methods: This was a secondary analysis of a prospective consecutive cohort of emergency department (ED) patients aged 18+ with opioid overdose presenting to nine sites within the ToxIC network from October 6, 2020 to August 17, 2021. Waste blood specimens were analyzed with liquid chromatography quadrupole time-of-flight mass spectrometry. The primary study outcome was total naloxone bolus dose administered. Secondary outcomes included endotracheal intubation, cardiac arrest, troponin elevation, and presenting vital signs. We performed t-test and chi-squared analyses to compare demographics and outcomes between groups.

Results: Of 378 enrollees, 207 (51.8\%) had psychostimulants and fentanyl present. Patients in the fentanyl only group were significantly older than the fentanyl/stimulant group (mean 45.2 years versus 40.6 years, $\mathrm{p}<$ $0.01)$. Patients in the fentanyl/stimulant group had significantly higher total naloxone dose requirements (mean total dose $3.56 \mathrm{mg}$ vs. 2.85 $\mathrm{mg}, \mathrm{p}=0.01)$. There was no significant difference in presenting vital signs or rates of intubation, cardiac arrest, and troponin elevation.

Conclusion: We identified a high rate of psychostimulants and fentanyl co-exposure. Patients in the fentanyl/stimulant group had significantly higher naloxone dose requirements, suggesting potential greater severity of overdose. Further, powered studies are needed to fully evaluate the impact of psychostimulant co-exposure on outcomes in fentanyl/fentalog overdose.

This research was performed in collaboration with the ACMT Toxicology Investigators Consortium

113. Methadone-induced Acute Cerebellitis Causing Isolated Ataxia in a Pediatric Patient

Michael D Simpson ${ }^{1,2}$, David B Appelbaum ${ }^{3}$, James D Whitledge ${ }^{1,2}$, Christopher J Watson ${ }^{1,2}$, Michele M Burns ${ }^{1,2}$

${ }^{1}$ Harvard Medical Toxicology, Boston, MA, USA. ${ }^{2}$ Boston Children's Hospital, Boston, MA, USA. ${ }^{3}$ Beth Israel Deaconess Medical Center, Boston, MA, USA

Background: Methadone-induced cerebellitis is a unique version of opioid-induced toxic leukoencephalopathy. Characteristic neuroimaging includes restricted diffusion of the bilateral cerebellar hemispheres on magnetic resonance imaging (MRI), but corresponding cerebellar symptoms are rare. Hypothesis: Methadone toxicity can cause both imaging and clinical evidence of cerebellar dysfunction.

Methods: This is a single patient case report; data/results were obtained by chart review. A 17-month-old male presented in respiratory arrest after reported exploratory exposure to an unknown amount of methadone. He was resuscitated, intubated, and transferred to tertiary care hospital intensive care unit (ICU). Urine drug screening was positive for methadone. Magnetic resonance imaging (MRI) of the brain was obtained to evaluate for hypoxic ischemic encephalopathy on hospital day two. He was extubated on hospital day five due to improving neurological and respiratory status. After extubation, he had persistent isolated truncal and lower extremity ataxia without other neurological deficits. Serum methadone and metabolite 2-ethylidene-1,5-dimethyl-3,3-diphenylpyrrolidine (EDDP) was measured on samples from initial arrival to the ICU and again on hospital day 10 , during which time the patient was still exhibiting ataxia. MRI was also repeated on day 10 .

Results: Serum methadone level at time of arrival to the ICU was $234 \mathrm{ng} /$ $\mathrm{mL}$, and serum EDDP level was $19 \mathrm{ng} / \mathrm{mL}$. MRI showed isolated decreased diffusivity in the cerebellum. Repeat testing on hospital day 10 showed an undetectable $(<10 \mathrm{ng} / \mathrm{mL})$ serum methadone and EDDP, and MRI showed resolution of the cerebellar findings. Despite this improvement in imaging, the patient continued to experience ataxia and gait disturbance requiring inpatient physical therapy. His gait improved, and he was independently ambulating by discharge on hospital day 20 .
Conclusion: Methadone overdose in children can cause persistent cerebellitis and ataxia despite normalization of neuro-imaging findings.

114. Tramadulterant: Tramadol Detected in Patient's Serum Following An Acute Opioid Overdose

Evan S Schwarz ${ }^{1}$, Kim Aldy ${ }^{2,3}$, Alison Meyn², Sharan Campleman ${ }^{2}$, Shao $\mathrm{Li}^{2}$, Alex Krotulski ${ }^{4}$, Barry Logan ${ }^{4,5}$, Paul Wax ${ }^{2,3}$, Jeffrey Brent ${ }^{6}$, Alex Manini ${ }^{7}$, On Behalf of the Toxicology Investigators Consortium (ToxIC) Fentalog Study Group

${ }^{1}$ Washington University School of Medicine, St. Louis, MO, USA.

${ }^{2}$ American College of Medical Toxicology, Phoenix, AZ, USA.

${ }^{3}$ University of Texas Southwestern Medical Center, Dallas, TX, USA.

${ }^{4}$ Center for Forensic Science Research and Education at the Fredric Rieders Family Foundation, Willow Grove, PA, USA. ${ }^{5}$ NMS Labs, Horsham, PA, USA. ${ }^{6}$ University of Colorado School of Medicine, Aurora, CO, USA. ${ }^{7}$ Icahn School of Medicine at Mount Sinai, New York, NY, USA

Background: In Europe, tramadol is alleged to be an adulterant of heroin, but this is poorly reported. Tramadol adulteration of the illicit opioid supply in the US could result in higher naloxone requirements and worse overdose severity.

Research Question: What are the naloxone requirements and overdose severities of ED patients with tramadol-adulterated illicit opioid overdose?

Methods: This is a case series from the Toxicology Investigators Consortium (ToxIC) Fentalog Study Group, an ongoing study with nine sites across the United States. Consecutive ED patients with a presumed acute opioid overdose and available waste blood samples were screened; pediatrics, prisoners, and those with non-toxicological diagnoses were excluded. Information including but not limited to demographics, substance use history, clinical course, vital signs, laboratory information, and disposition were collected following a chart review. Residual blood samples were obtained from all included patients and sent for toxicological confirmation via liquid chromatography quadrupole time-of-flight mass spectrometry for the presence of over 900 substances, including novel psychoactive substances and metabolites.

Results: Between 10/2000-10/2021, 1006 patients were screened, 378 met inclusion criteria, and 67 (18\%) from six hospitals tested positive for tramadol. Of these, co-exposure to fentanyl occurred in 95\%. Fortythree $(64 \%)$ were male; ages ranged $20-75$. Only five reported taking tramadol. Forty-eight $(72 \%)$ received naloxone, with $26(39 \%)$ receiving a second dose, and eight (12\%) a third dose. Bolus dosing ranged from 0.04-6 $\mathrm{mg}$ and three $(4.5 \%)$ required a naloxone infusion. Only two died, but five were intubated and seven received CPR.

Conclusion: Tramadol was detected in $18 \%$ of our cohort following a presumed acute opioid overdose. Few realized they were exposed to tramadol. Repeat naloxone bolus requirements were common, and overdose severity was higher than expected. While the hospitals participating in the study group are geographically diverse, they may not be representative.

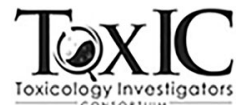

This research was performed in collaboration with the ACMT Toxicolog Investigators Consortium

115. Identification of a Novel Opioid, N-piperidinyl etonitazene, in Patients with Suspected Opioid Overdose

Diane P Calello ${ }^{1}$, Mohamed Jefri ${ }^{1}$, Tuyet-Anh Nguyen ${ }^{1}$, Kim Aldy ${ }^{2,3}$, Sharan Campleman ${ }^{2}$, Alex Krotulski ${ }^{4}$, Barry Logan ${ }^{5,4}$, Paul Wax ${ }^{2,3}$, Jeffrey Brent ${ }^{6}$, Alex Manini ${ }^{7}$, On Behalf of the Toxicology Investigators Consortium (ToxIC) Fentalog Study Group

${ }^{I}$ Rutgers New Jersey Medical School, Newark, NJ, USA. ${ }^{2}$ American College of Medical Toxicology, Phoenix, AZ, USA. ${ }^{3}$ University of Texas Southwestern Medical Center, Dallas, TX, USA. ${ }^{4}$ Center for Forensic 
Science Research and Education at the Fredric Rieders Family Foundation, Willow Grove, PA, USA. ${ }^{5}$ NMS Labs, Horsham, PA, USA. ${ }^{6}$ University of Colorado School of Medicine, Aurora, CO, USA. ${ }^{7}$ Icahn School of Medicine at Mount Sinai, New York, NY, USA

Background: Novel synthetic opioids (NSOs) in the illicit opioid supply present an ongoing public health problem, such as the "nitazene" group of synthetic opioids. 2-benzylbenzimidazole opioids, also termed "nitazene" compounds, have been reported to exhibit potencies at the mu opioid receptor exceeding that of fentanyl. Some nitazenes have emerged in the illicit drug market with resultant overdoses, however N-piperidinyl etonitazene has not previously been identified. We describe three patients with N-piperidinyl etonitazene, a compound not previously reported in human exposure, detected after suspected opioid overdose.

Research Question: This study aims to evaluate the clinical effects and outcomes of a recently emerged NSO.

Methods: This case series is derived from the Toxicology Investigators Consortium (ToxIC) Fentalog Study, an ongoing multicenter prospective cohort. Consecutive adult patients with suspected acute opioid overdose were screened for enrollment, clinical data was collected, and waste blood specimens were analyzed via liquid chromatography quadrupole time-offlight mass spectrometry. Exclusion criteria was pediatrics, prisoners, and those with burns/trauma.

Results: Between 10/6/20-10/31/21, 412 met inclusion criteria, and three tested positive for N-piperidinyl etonitazene at one site (New Jersey) over a period of three days in July 2021. Ages ranged from 33-55, and two were male. Two reported using cocaine, and one reported heroin and alprazolam. One case had no other opioids present, and two also contained fentanyl. All three patients received initial naloxone doses with improved mental status, and two received subsequent doses for opioid toxicity recurrence. One patient had pneumonia and ICU admission, one was discharged from the emergency department, and one used additional drugs after arrival necessitating admission for naloxone infusion. All were discharged without organ damage or sequelae.

Conclusion: This represents the first clinical report of an outbreak of $\mathrm{N}$ piperidinyl etonitazene. Ongoing analytical testing of opioid overdoses may detect growth of this emerging class of compounds.

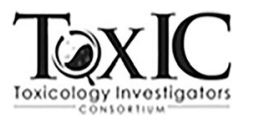

This research was performed in collaboration with the ACMT Toxicology Investigators Consortium.

116. Can Reddit Posts Reveal Insurance Related Barriers to Accessing Medication for Opioid Use Disorder?

Whitney Bremer ${ }^{1}$, Abeed Sarker ${ }^{1}$, Jeanmarie Perrone ${ }^{2}$

${ }^{1}$ Emory University, Atlanta, GA, USA. ${ }^{2}$ University of Pennsylvania, Philadelphia, PA, USA

Background: Medications such as buprenorphine, methadone and naltrexone are evidence-based treatments for opioid use disorder (OUD). Many people with OUD face barriers to treatment that are related to health insurance. Our objective was to study and characterize insurance-related barriers to OUD treatment using social media data from Reddit.

Research Question: Can Reddit serve as a resource to study insurancerelated barriers for patients seeking medication for OUD?

Methods: This was an observational study involving posts from the platform Reddit, specifically three OUD related forums (subreddits): $r /$ suboxone, $r$ /Methadone, and r/naltrexone subreddits. Posts that mentioned 'insurance' were extracted from these subreddits $(\mathrm{n}=378)$, and manually reviewed. Posts unrelated to barriers were excluded from detailed analyses $(n=179)$. The remaining posts $(n=199)$ were analyzed and grouped into five categories based on the type of barrier (uninsured, partial insurance coverage, fear of losing insurance, switched treatments due to insurance changes, and logistical barriers). The category with the most numerous posts, "logistical barriers" were further analyzed qualitatively.

Results: Among 199 posts that were posted between 7/2/2013 and 8/2/ 2021, the proportions for methadone, suboxone and naltrexone were $44.4 \%, 47.6 \%$, and $7.9 \%$, respectively. A total of $12.4 \%$ mentioned not being insured, $12.7 \%$ mentioned being insured but only partially covered, $1.9 \%$ expressed fear of losing insurance, $1.3 \%$ mentioned the need to switch treatments because of insurance barriers, and $18.3 \%$ mentioned obstacles or frustrations surrounding logistical issues. Logistical issues included, but were not limited to, the length of time between insurance approval and treatment, stigma surrounding substance use and OUD, and lack of medication facilities in certain geographic locations.

Conclusion: Reddit can be used as an insightful resource for understanding individual, detailed self-reported insurance-related barriers to accessing medication for OUD. Our future work will focus on characterizing additional barriers.

117. Relative Frequency of Polydrug Exposures Involving Opioids and Sedative-Hypnotic Agents in the ToxIC Registry by Race/Ethnicity: 2015-2020

Sharan L Campleman ${ }^{1}$, Shao $\mathrm{Li}^{1}$, Kim Aldy ${ }^{1,2}$, Jeffrey Brent ${ }^{3}$, Paul $\mathrm{Wax}^{1,2}$, On Behalf of the Toxicology Investigators Consortium (ToxIC) ${ }^{1}$ American College of Medical Toxicology, Phoenix, AZ, USA.

${ }^{2}$ University of Texas Southwestern Medical Center, Dallas, TX, USA.

${ }^{3}$ University of Colorado School of Medicine, Aurora, CO, USA

Background: Race/ethnicity-related differences in the identification of substance use patterns may interfere with the ability to fully identify and intervene in the social determinants contributing to health risk.

Research Question: To what degree can variation in race/ethnicity among cases of polydrug exposures related to opioid and sedative use be identified in a medical toxicology-based surveillance system?

Methods: An analysis of cases reported to the ToxIC Core Registry (extract version 2021_sep9.1) between January 1, 2015, and December 31,2020 , with at least one agent reported were included in this analysis. The relative occurrence of multiple drug exposures, including for opioids and/or sedatives, were quantified across the eight race categories plus unknown race. STATA $^{\odot}$ was used for descriptive analysis.

Results: Over this six-year period, 39,104 of 45,056 cases $(86.8 \%)$ reported at least one agent related to a toxic exposure. Almost one-third of these cases reported more than one agent, of these $42.0 \%$ included an opioid, sedative, or both ( $\mathrm{n}=5,416,13.9 \%$ total). A small subset of cases reported at least one of each class $(n=1,001)$ reflecting $7.8 \%$ of all multiagent exposures. The relative frequency of polydrug exposures across specified race groups ranged between $22.8 \%-33.8 \%$ but was highest in the "Unknown" race category (36.4\% of cases reporting any agent). In the mixed opioid-sedative exposure cases, small numbers limited frequency comparisons $(<5$ cases for Native Hawaiian/Pacific Islander, Mixed, Other categories). The risk ratios (RR) for NonHispanic White and Unknown race groups in joint exposure cases were significantly higher, $\mathrm{RR}=1.16(95 \% \mathrm{CI} 1.11-1.22)$ and $\mathrm{RR}=1.22(95 \%$ $\mathrm{CI}=1.05-1.43)$, respectively.

Conclusion: The ToxIC Registry does provide a resource for breaking down the distribution of polydrug cases in a real-world practice-based surveillance system; however, the relative frequency of cases reporting race as "Unknown" limits the ability to fully understand exposure differences based on race/ethnicity.

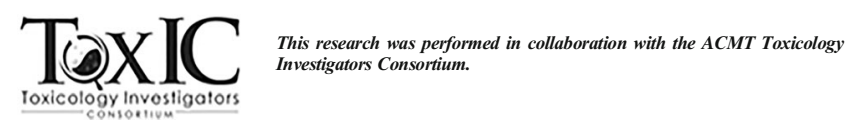

118. Race, Ethnicity, and Age Distribution of Opioid Exposures Seen by Medical Toxicologists 
Jennifer S Love ${ }^{1}$, Sharan Campleman ${ }^{2}$, Paul Wax ${ }^{2,3}$, Jeffrey Brent ${ }^{4}$, Kim Aldy $^{2,3}$, On Behalf of the Toxicology Investigators Consortium (ToxIC) ${ }^{1}$ Icahn School of Medicine at Mount Sinai, New York, NY, USA. ${ }^{2}$ American College of Medical Toxicology, Phoenix, AZ, USA. ${ }^{3}$ University of Texas Southwestern Medical Center, Dallas, TX, USA. ${ }^{4}$ University of Colorado School of Medicine, Aurora, CO, USA

Background: Traditional poisoning data sources, such as the National Poison Data System, do not collect information on race and ethnicity. Given that disparities may impact opioid use, it is important to understand race/ethnicity and age in opioid-related exposures.

Research Question: Describe race/ethnicity and age in opioid-related exposures.

Methods: This is a retrospective analysis of the ToxIC Core Registry between 2014-2021. Cases with at least one opioid exposure agent were included. Opioids reported were grouped into fentanyl/heroin, commonly prescribed opioids, medications for opioid use disorder, and other opioid agents. SAS ${ }^{\circledR}$ was used for statistical analysis.

Results: Between 2014-2021, 7,311 of 60,564 cases (12.1\%) had an opioid exposure. For fentanyl/heroin exposure, frequency by race/ethnicity was: Non-Hispanic White $50.2 \%$, Black/African American 17.6\%, Hispanic 10.5\%, Asian 0.76\%, American Indian/ Alaska Native (AIAN) $0.43 \%$, Native Hawaiian $0.03 \%$, mixed race $0.43 \%$, Other $0.03 \%$, and unknown $42.9 \%$ ( $\mathrm{p}<0.001)$. For prescription opioid exposure, frequency was Non-Hispanic white $52.46 \%$, Black/ African American 11.83\%, Asian 9.95\%, Hispanic 8.6\%, AIAN 1.4\%, mixed race $0.12 \%$, Native Hawaiian $0.35 \%$, other $0.47 \%$, and unknown $14.9 \%(\mathrm{p}<0.001)$. For fentanyl/heroin exposure, frequency by age was $1.43 \%$ age $<$ two years, $0.5 \%$ ages $2-6,0.24 \%$ ages $7-12,4.83 \%$ ages $13-$ $18,90.7 \%$ ages $19-65,1.76 \%$ ages $66-89$, and $0.03 \%$ ages $89+(\mathrm{p}<$ $0.001)$. For prescription opioid exposure, $3.28 \%$ ages $<$ two years, $0.82 \%$ ages $2-6,2.22 \%$ ages $7-12,18.6 \%$ ages $13-18,63.8 \%$, ages $19-$ $65,10.2 \%$ ages $66-89$, and $0.35 \%$ ages $89+(\mathrm{p}<0.001)$.

Conclusion: For ToxIC Registry patients, differences in rates of exposure to opioids (fentanyl/heroin, prescription opioids) exist by race/ethnicity and age but may be confounded by participating site location and severity of exposure necessitating toxicologist consultation. Evaluation of race/age disparities can inform future studies on contributing factors and guide targeted harm-reduction interventions to at-risk groups.

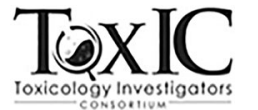

This research was performed in collaboration with the ACMT Toxicology Investigators Consortium.

119. Deaths Involving Xylazine-Adulterated Heroin in Michigan Andrew M King ${ }^{1,2}$, Varun Vohra ${ }^{2}$

${ }^{1}$ Wayne State University, Detroit, MI, USA. ${ }^{2}$ Michigan Poison and Drug Information Center, Detroit, MI, USA

Background: Xylazine is a central alpha-2 agonist used for analgesia, anesthesia, and sedation in veterinary medicine and not approved for human use. Clinical effects include hypotension, bradycardia, and central nervous system and respiratory depression. It has been detected as an adulterant in US illicit opioid supplies since the early 2000s. As such, combination use may potentiate opioid toxicity, placing users at increased risk of fatality. A recent series of deaths involving xylazine have been reported in western Michigan suggesting a potential increase in exposure prevalence statewide.

Hypothesis: Xylazine is present in Michigan's illicit opioid and/or amphetamine supplies.

Methods: This is a case series of medical examiner postmortem reports of xylazine-associated deaths with confirmed levels from January, 2020 to April, 2021 across six counties in western Michigan. Data abstracted included demographics, postmortem toxicology results, and cause and manner of death.
Results: Sixteen postmortem cases of xylazine-associated deaths were obtained. The mean age was 38 years (range, 24 to 60 years) with the majority involving Caucasian males $(69 \%)$. All deaths were deemed accidental and caused by the toxic effects involving multiple substances, including xylazine. Range of postmortem xylazine concentrations was 6.2 to $200 \mathrm{ng} / \mathrm{mL}$. All cases involved fentanyl (concentration range, 4.9 to $140 \mathrm{ng} / \mathrm{mL}$ ), $15 / 16$ detected 4-ANPP, and more than half involved amphetamine ( 9.8 to $500 \mathrm{ng} / \mathrm{mL}$ ) and methamphetamine (49 to 2600 $\mathrm{ng} / \mathrm{mL}$ ). A Pearson correlation coefficient demonstrated a positive correlation between xylazine and fentanyl concentrations $(r=0.47)$. Four cases detected morphine (10 to $140 \mathrm{ng} / \mathrm{mL}$ ) and two detected 6-monoacetylmorphine (2.2 to $3.1 \mathrm{ng} / \mathrm{mL}$ ).

Conclusion: This case series examines postmortem toxicology trends in xylazine detection. The correlation between xylazine and fentanyl (+/- 4ANPP) suggest xylazine is added to illicitly manufactured fentanyl. This study supports the need for more statewide resources for xylazine detection during routine toxicology testing.

120. Phencyclidine-Associated Laryngospasm: A Case Report Laura B Roper, Steven J Walsh, J Michael Kowalski

Einstein Medical Center, Philadelphia, PA, USA

Background: Phencyclidine (PCP) is a member of the arylcyclohexylamine class of dissociative anesthetic drugs, which also includes ketamine. Laryngospasm is a rare, unpredictable, and potentially life-threatening complication of ketamine use; to our knowledge, laryngospasm has not been reported with PCP use.

Hypothesis: Are patients intoxicated with PCP at risk of laryngospasm? Methods: This is a case report. A 44-year-old man with a history of polysubstance use and hypertension presented with altered mental status. Due to complete unresponsiveness and copious secretions, the patient was immediately intubated for airway protection. The intubation was difficult, requiring several intubation attempts by the emergency medicine physicians and the anesthesiologist due to laryngospasm. Urine drug screen (UDS) was obtained as part of routine clinical care. A comprehensive toxicology panel was ordered for confirmatory testing on serum left over from the initial blood draw. There was no urine sample left from the initial presentation for confirmatory testing.

Results: UDS was positive for PCP and was otherwise negative. Confirmatory testing of serum was positive for PCP and negative for ketamine and norketamine. No other substances were subsequently detected by liquid chromatography-mass spectrometry. The patient was liberated from mechanical ventilation after 36 hours. He recovered fully and was discharged three days after symptom onset.

Conclusion: PCP-intoxicated patients are at risk of developing laryngospasm, much like patients receiving ketamine. This phenomenon may be due to copious secretions eliciting an involuntary closure of the vocal cords and glottis in an attempt to protect the airway. These agents are thought to cause hypersalivation due to stimulation of muscarinic receptors, which occurs at significantly higher concentrations than needed to inhibit NMDA receptors.

121. Clonazolam, a Designer Benzodiazepine, Presents Unique Challenges in Withdrawal Compared to Traditional Benzodiazepines Christopher F Dion ${ }^{1}$, Krista R Labruzzo ${ }^{1}$, Meghan B Spyres ${ }^{1,2}$

${ }^{1}$ Banner - University Medical Center Phoenix, Phoenix, AZ, USA

${ }^{2}$ University of Arizona College of Medicine Phoenix, Phoenix, AZ, USA

Background: Clonazolam is a high potency designer benzodiazepine (DBZD) readily available for purchase on the internet. Due to its high potency, management of withdrawal from clonazolam may be challenging and is not well described.

Hypothesis: DBZD withdrawal may require high dose $\mathrm{GABA}_{\mathrm{A}}$ agonist treatment.

Methods: This is a single patient chart review. A 39-year-old man with a history of anxiety and DBZD dependence presented to the ED with 
benzodiazepine withdrawal. He reported taking $3.75 \mathrm{mg} /$ day of clonazolam (equivalent to $188 \mathrm{mg}$ diazepam), sold as a $5 \mathrm{mg} / 1 \mathrm{~mL}$ liquid on the internet, until the medication became unavailable. He substituted flucotizolam, a different DBZD sold as a $0.5 \mathrm{mg} / 1 \mathrm{~mL}$ liquid, without desired effect. He presented in acute benzodiazepine withdrawal and was managed with escalating doses of phenobarbital in the hospital.

Results: The patient was successfully transitioned from $3.75 \mathrm{mg} /$ day clonazolam to $130 \mathrm{mg}$ TID phenobarbital during a five-day inpatient hospitalization. He received $1105 \mathrm{mg}$ of phenobarbital in the first 26 hours $(13.1 \mathrm{mg} / \mathrm{kg})$. Phenobarbital level was $37.6 \mu \mathrm{g} / \mathrm{dL}$ on discharge, which is near the upper limit of the therapeutic range. He was able to tolerate phenobarbital without sedation and required frequent redosing to obtain clinical effect. Testing confirmed a serum clonazolam level of 6.4 $\mathrm{ng} / \mathrm{mL}$ on admission. Testing for other conventional and DBZDs was negative; flucotizolam levels were not available. The patient continues a phenobarbital taper two months after discharge. His pharmacogenomics profile revealed normal CYP3A4 metabolism and rapid CYP2C19 metabolism. Clonazolam is primarily metabolized by CYP3A4 and flucotizolam is likely metabolized by CYP2C19, which may have contributed to the diminished effect of flucotizolam.

Conclusions: High potency DBZDs are easily accessible, yet supply can be disrupted suddenly. Withdrawal can require higher than expected doses of $\mathrm{GABA}_{\mathrm{A}}$ agonist compared to traditional benzodiazepines.

\section{Novel Benzodiazepine Ingestion Associated with Sinus Bradycardia and Premature Ventricular Contractions (PVC) in Overdose \\ Alaina Royalty, Isaac Shaw, Matthew Eisenstat \\ University of Louisville, Louisville, KY, USA}

Background: Benzodiazepines have at times been associated with cardiovascular effects such as bradycardia and dysrhythmias. Novel illicit benzodiazepines are becoming a contaminant of concern both alone and in combination with opioids.

Hypothesis/Research Question: To our knowledge no illicit novel benzodiazepines have been associated with bradycardic dysrhythmias. We present a case report of likely etizolam overdose with bradycardia and PVCs.

Methods: Forty-four year-old male with past medical history notable only for substance use disorder presented to the emergency department following an overdose. The patient was reported to have taken one gram of heroin and $22 \mathrm{mg}$ of "Xanax" (that had been illicitly purchased) orally in an effort to avoid police detection. He was given a total of eight mg IN naloxone prior to arrival to the ED. He was subsequently given four $\mathrm{mg}$ IV naloxone in the emergency department after he remained obtunded, with minimal respiratory effect. He had minimal response to naloxone and was intubated. In the ED EKG was notable for bigeminy with a rate in the 40s-50s associated with a prolonged QTc. Patient was found to have a quantitative etizolam level of $160 \mathrm{ng} / \mathrm{mL}$ and its major metabolite alphahydroxyetizolam (at $100 \mathrm{ng} / \mathrm{mL}$ ). No other tested NPS were detected including a large number of illicitly manufactured fentanyls (IMF). The patient had intermittent episodes of bradycardia following his overdose that resolved by hospital day three. QTc remained greater than 600 for several days but was 460 by the second week of his stay. On the last day of his hospital stay his EKG was normal sinus rhythm with a rate in the 70s and normalized QTc.

Conclusion: Etizolam may be associated with bradycardia and other EKG changes. Further case reports, retrospective studies and perhaps animal studies would be required to solidify this link.

123. Gamma Hydroxybutyrate Intoxication Presenting with Elevated Anion Gap Metabolic Acidosis

Justin A Seltzer $^{1,2}$, Bryan Corbett ${ }^{1}$, Richard F Clark ${ }^{1}$

${ }^{1}$ University of California, San Diego Division of Medical Toxicology, Department of Emergency Medicine, San Diego, CA, USA. ${ }^{2}$ VA San Diego Healthcare System, San Diego, CA, USA
Background: Gamma hydroxybutyrate (GHB) is a sedative-hypnotic structurally related to gamma aminobutyric acid (GABA). It is also an isomer of beta hydroxybutyrate (BHB), which is partly responsible for the anion gap metabolic acidosis (AGMA) characteristic of ketoacidosis. Despite similarities, GHB is not a well described cause of AGMA, with only a few published reports.

Methods: This is a single patient chart review. A 53-year-old male with depression presented to an outside hospital after being found unresponsive. He was hypoxic, bradycardic, and obtunded, requiring intubation for airway protection. He was then transferred for specialty care.

Results: Outside hospital laboratory evaluation one hour after time of ingestion showed a pH of 6.992 and partial pressure of $\mathrm{CO} 2$ (PCO2) of $51 \mathrm{~mm} \mathrm{Hg}$. Serum bicarbonate was $13 \mathrm{mmol} / \mathrm{L}$, with an anion gap of 30 . Lactic acid was $2.6 \mathrm{mmol} / \mathrm{L}$. EKG showed sinus bradycardia. Bicarbonate and dopamine infusions were started prior to transfer, for acidosis and bradycardia respectively. On ICU arrival seven hours later, he was unresponsive without sedation with miotic pupils. The $\mathrm{pH}$ was 7.28 and normalized two hours later without additional intervention. Lactic acid and BHB were normal. Salicylate and toxic alcohol levels were undetectable. An endoscopy, performed for reported caustic ingestion, was unremarkable.

The patient awoke around 12 hours after ingestion and was extubated the next day. He admitted intentionally overdosing on GHB for self-harm. He was then transferred to the psychiatry inpatient unit.

Conclusion: Though the mechanism of GHB-related AGMA is not fully understood, GHB has a similar $\mathrm{pKa}$ as BHB and, in large overdoses, likely behaves similarly. However, the BHB assay does not appear to cross-react. In an unresponsive patient with an AGMA of unclear etiology, GHB should be considered.

124. Antimuscarinic or Asleep: A Comparison of Antimuscarinic Effects Between Hydroxyzine and Diphenhydramine Poisoned Patients Mark W Simon ${ }^{1}$, Kennon J Heard ${ }^{2}$, On Behalf of the Toxicology Investigators Consortium (ToxIC)

${ }^{I}$ Denver Health Medical Center, Denver, CO, USA. ${ }^{2}$ University of Colorado Hospital, Aurora, CO, USA

Background: First-generation $\mathrm{H}_{1}$ antihistamines, such as hydroxyzine and diphenhydramine, are generally categorized as having antimuscarinic findings. However, hydroxyzine has a much lower affinity for the human muscarinic receptor than many other medications in this class, such as diphenhydramine. Hypothesis: the objective of this study was to compare the rates of antimuscarinic effects in hydroxyzine and diphenhydramine exposures.

Methods: This is a retrospective, cohort analysis that compared hydroxyzine and diphenhydramine exposures reported to the Toxicology Investigators Consortium (ToxIC). The study population included all patients with single-substance ingestions between January 1, 2000, and December 31,2020. To determine the relative antimuscarinic effects and overall toxicity of each medication, we measured the percentage and relative risk (RR) of associated findings. We calculated $95 \%$ confidence intervals for the RR and compared percentages using Chi-squared testing. Results: There were 134 hydroxyzine and 1,462 diphenhydramine ingestions reported to ToxIC during the study period. The median age was 17 years (IQR 14-29 years) and 62\% were female. An anticholinergic toxidrome was reported less commonly ( $\mathrm{RR}=0.37,95 \% \mathrm{CI}: 0.27$ 0.52 ) for hydroxyzine $(21 \%)$ than diphenhydramine $(57 \%)$. Other common anticholinergic effects were also less common with hydroxyzine including agitation $(\mathrm{RR}=0.40,95 \% \mathrm{CI}: 0.26$ to 0.61$)$, delirium $(\mathrm{RR}=$ $0.48,95 \%$ CI: $0.34-0.67$ ), hallucinations ( $R R=0.20,95 \%$ CI: $0.09-0.44$ ), and treatment with physostigmine $(\mathrm{RR}=0.40,95 \% \mathrm{CI}$ : $0.22-0.74)$ and benzodiazepines ( $\mathrm{RR}=0.38,95 \% \mathrm{CI}: 0.26-0.55)$. CNS depression was more common with hydroxyzine $(\mathrm{RR}=1.6,95 \% \mathrm{CI}: 1.2-2.2)$. Mechanical ventilation was less common for hydroxyzine ( $0 \%$ vs. $7.7 \%)$. There were eight deaths from diphenhydramine $(0.55 \%)$ and one death from hydroxyzine $(0.76 \%)$. However, it was unknown if that 
patient was exposed to hydroxyzine and signs and symptoms were deemed "unlikely" to be toxicologically related.

Conclusion: This study shows that patients who ingested hydroxyzine have significantly less antimuscarinic effects than patients who ingested diphenhydramine, a prototypical first-generation $\mathrm{H}_{1}$ antihistamine.

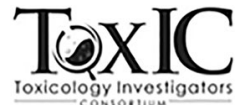

This research was performed in collaboration with the ACMT Toxicology Investigators Consortium.

125. Results of Urine Drug Screens for Emergency Department Patients Presenting with Psychiatric Emergencies

Simran Koura ${ }^{1}$, Joshua Masdon ${ }^{2}$, Avian White ${ }^{1}$, Kori Brewer $^{2}$, William Meggs $^{2}$

${ }^{1}$ East Carolina University Brody School of Medicine, Greenville, NC, USA. ${ }^{2}$ Brody School of Medicine, Emergency Medicine, Greenville, $N C, U S A$

Background: Patients with psychiatric emergencies routinely have urine drug screens to aid in diagnosis and treatment. Since specific illicit drugs are often associated with psychiatric syndromes, correlations between drugs and psychiatric diagnosis may be of interest to toxicologists.

Research Question: Are patients presenting to a hospital with psychiatric emergencies likely to have positive urine drug screens, and if so, is there a correlation between drug and diagnosis?

Methods: A retrospective chart review of patients presenting with psychiatric emergencies was conducted with IRB approval. One thousand charts with diagnoses of anxiety, depression, schizophrenia, ADHD, bipolar disorder, alcohol abuse, or schizoaffective disorder were reviewed. Data collected included demographics, tobacco abuse, chief complaint, suicide attempt, psychiatric diagnoses, urine drug screen (barbiturates, benzodiazepines, cocaine, opiates, amphetamines, cannabinoids, phencyclidine, MDMA, and fentanyl) and ethanol results. IBM SPSS Program 26.0 was used to conduct statistical analyses. A chi-square test was run to examine the relationship between substances of abuse and psychiatric diagnoses.

Results: Fifty-eight percent of patients presenting with psychiatric emergencies had a drug screen positive for at least one substance. Cannabis was the most common positive drug screen across psychiatric diagnoses except alcohol use in ADHD. Of 245 patients with schizoaffective disorder, $69(28 \%)$ were positive for tetrahydrocannabinol (THC) and 48 (20\%) were positive for cocaine. Of 225 patients with depression, 59 (29\%) were positive for THC and $33(15 \%)$ were positive for cocaine. No statistical significant correlations were found between psychiatric diagnosis and positive drug screen.

Conclusion: Positive drug screens were common among patients presenting with psychiatric emergencies. No statistically significant correlations were found between psychiatric diagnosis or other parameters. Since drug screens correlate with recent drug use but not intoxication or chronic use, a prospective study might find correlations.

126. Impact of Asynchronous Substance Use Treatment Training on EM Board Certification Scores

Carrie A Dickenson ${ }^{1}$, Kevin Joldersma ${ }^{2}$, Lewis Nelson ${ }^{3}$, Andrew Stolbach $^{4}$, Paul M Wax ${ }^{1}$

${ }^{I}$ American College of Medical Toxicology, Phoenix, AZ, USA ${ }^{2}$ American Board of Emergency Medicine, East Lansing, MI, USA. ${ }^{3}$ Rutgers University, Newark, NJ, USA. ${ }^{4}$ Johns Hopkins, Baltimore, Maryland, USA

Background: Although substance use disorders (SUD) are part of the American Board of Emergency Medicine (ABEM) core content, there is no single standardized SUD training for residents. Under a grant from Substance Abuse and Mental health Services Administration
(SAMHSA), the American College of Medical Toxicology (ACMT) created the Substance Use Screening and Treatment Approach in the ED (SUSTAIN-ED) Classroom, which offers online SUD learning to emergency medicine (EM) residents. Between 2020 and 2021, this curriculum was made available to $22 \mathrm{EM}$ residency programs. As part of the grant deliverables, ACMT partnered with the American Board of Emergency Medicine (ABEM) to develop test items from the curriculum to be included in the 2021 Emergency Medicine In-training Examination (ITE). Research Question: Can an asynchronous training curriculum on the screening, assessment, and treatment of SUD improve EM physician performance on ITE test items on this topic?

Methods: We submitted to ABEM 28 test items based on the SUSTAINED curriculum. After review by editors, four test items were included in the 2021 EM In-training examination. Following the exam, ABEM compared In-training performance on those items between residents with access to the curriculum and those without access.

Results: One thousand eighty one examinees came from programs that had access to the SUSTAIN-ED curriculum and 7782 came from sites that did not have access. On three of the four test items, examinees from sites with access to the curriculum performed $7-10 \%$ better than those without access. On the fourth test item there was no difference between the two groups.

Conclusion: EM resident physicians that had access to ACMT SUSTAIN-ED training scored higher on test items relating to substance use screening and treatment than those that did not have access. The addition of asynchronous training to standard emergency medicine didactic curriculum may enhance residents' acquisition of knowledge as measured on ITE performance.

\section{Tiletamine-Zolazepam Abuse and Withdrawal in a Veterinary Health Worker \\ Erik S Fisher \\ Prisma Health-Upstate, Greenville, SC, USA. University of South Carolina School of Medicine Greenville, Greenville, SC, USA}

Background: Healthcare professionals are at increased risk of substance use disorders compared to the general population. There is a paucity of literature regarding substance use disorders in veterinary healthcare workers, but the misuse of veterinary medications in this population is increasingly recognized. We report a case of tiletamine-zolazepam (Telazol ${ }^{\circledR}$ ) abuse to treat opioid use disorder.

Methods: A 35-year-old male veterinary technician presented to an Emergency Department complaining of tremor and myoclonus. He admitted to using the veterinary anesthetic Telazol®, a mixture of tiletamine, an arylaminocycloalkanone dissociative anesthetic related to ketamine, and zolazepam, a pyrazolodiazepinone sedative-hypnotic structurally related to benzodiazepines. He had a history of opioid use disorder on methadone maintenance, read about the potential of ketamine as a treatment, and began using Telazol 2-3 times daily starting two weeks before presentation. He developed rest and intention tremors one week into his use that increased during periods of abstinence. He began using unspecified doses of parenteral diazepam and midazolam to treat the tremors, but they worsened as his Telazol supply was depleted. On the day of presentation, the tremors became too severe to conceal at work and he could not obtain intravenous access for his last Telazol dose.

In the ED he was tachycardic to 115 and hypertensive to $151 / 104$. He experienced visual hallucinations prior to ED arrival, but none in the hospital. Exam revealed a clear sensorium, resting tremors affecting all extremities that worsened with intention and frequent myoclonus involving the upper extremities. UDS detected benzodiazepines with diazepam metabolites on confirmation.

He was admitted to intensive care and received $160 \mathrm{mg}$ of diazepam and $9 \mathrm{mg}$ of lorazepam over 72 hours for tremor, hypertension, and tachycardia. Tremors improved but did not resolve and he was discharged on a diazepam taper. 
Discussion: Substance abuse among physicians and nurses garners a great deal of attention but other healthcare professionals are also at increased risk. Veterinary healthcare workers may attempt to treat their own medical conditions using pharmaceuticals not approved for human consumption. The prevalence of veterinary medication abuse is underreported, and toxicity or withdrawal may not be recognized.

Conclusion: Clinicians should consider the possibility of veterinary pharmaceutical use in this population. Healthcare workers may not be forthcoming about efforts to manage their own conditions with agents available in their places of employment and a thorough history is needed to uncover these cases.

128. An Outbreak of Synthetic Cannabinoid-Associated Coagulopathy: Characteristics and Outcomes of Patients

Ophir Lavon

Carmel Medical Center, Haifa, Israel. Technion-Israel Institute of Technology, Haifa, Israel

Background: During September and October 2021, an outbreak of synthetic cannabinoid-associated coagulopathy cases in the Haifa district in northern Israel has occurred.

Research question: What were the characteristics and outcomes of hospitalized synthetic cannabinoid-associated coagulopathy patients?

Methods: Single center retrospective chart review study of patients, who were diagnosed with synthetic cannabinoid-associated coagulopathy, was performed. Demographic and clinical data were retrieved from electronic medical records and analyzed.

Results: Nine patients were included in the study. Eight (89\%) were male. Average age was 33.2 years (range 19-43). Most patients $(\mathrm{n}=$ 6, 67\%) were known drug abusers. Eight (89\%) confirmed recent use of cannabinoids. All presented with some bleeding manifestations: rectal bleeding $(n=4)$, hemothorax $(n=2)$, superficial hematoma $(\mathrm{n}=2)$, and hematuria $(\mathrm{n}=1)$. Average INR on admission was 14 (PT $189 \mathrm{sec}$ ) with a range of 3-22, (PT range 39-303 sec). In six (67\%) patients, hemoglobin on admission was low (5.1-11 gr\%). Coagulation factors products were administered to five patients: prothrombin complex concentrate (PCC) in three patients and fresh-frozen plasma (FFP) in two. Vitamin $\mathrm{K}_{1}$ (phytonadione) was administered in all patients; initial dose, given IV, ranged from 10 to $30 \mathrm{mg}$. Maintenance dose of vitamin $\mathrm{K}_{1}$ was 10-30 mg BID orally. Two patients required thoracic surgery. Duration of hospitalization was six days on average (range 3-13). All patients regained normal INR with long term Vitamin K treatment. Samples of the synthetic cannabinoid, provided by a few of the poisoned patients were analyzed by the Israel Police Forensics Laboratory, and found to be adulterated with brodifacoum, a highly potent 4hydroxycoumarin vitamin $\mathrm{K}$ antagonist anticoagulant rodenticide.

Conclusion: Coumarin adulteration of synthetic cannabinoids can lead to a mass toxicological event with severe clinical manifestations and significant public health consequences. High alert and prompt use of high dose vitamin $\mathrm{K}_{1}$ replacement therapy are essential and effective for improved outcomes.

\section{The Wolf in Sheep's Clothing: Caffeine}

Mikayla Y Hamilton, Tyler M Miklovic, Mark L Riddle, Alexander F Barbuto

Carl R. Darnall Army Medical Center, Fort Hood, TX, USA

Background: Caffeine is the world's most widely consumed psychoactive drug. It is ubiquitous and increasingly available in a multitude of different formulations. Despite widely penetrating all bodily tissues with profound physiologic effects, it is rarely associated with toxicity. Caffeine is a competitive antagonist of all four adenosine receptor subtypes and acts as a phosphodiesterase inhibitor. Clinically, adenosine antagonism results in medullary-driven tachypnea, increased gastric acid secretion, and refractory seizures. Furthermore, phosphodiesterase inhibition manifests as functional beta-agonism due to increased intracellular calcium. One of caffeine's metabolites, theophylline, is a pharmaceutical methylxanthine, infamous for its narrow therapeutic window and adverse effects of tachyarrhythmia, seizure, and CNS excitation. Hypothesis: Benzodiazepines and beta blockers can be used to treat sustained ventricular arrhythmia with a pulse.

Methods: This is a case report of a caffeine intoxication at a military hospital.

Results: A 20-year-old presented via EMS 30 minutes after an intentional ingestion of caffeine tablets totaling 16 grams with associated altered mental status, repeated vomiting, profuse diaphoresis, and chest pain. The patient initially presented in narrow complex sinus tachycardia that shortly after arrival developed into symptomatic polymorphic ventricular tachycardia with a pulse. They were given IV medical therapy including two mg lorazepam, two g magnesium, $20 \mathrm{mg}$ labetalol, and two L normal saline with successful sustained conversion to sinus rhythm. A repeat dose of one mg lorazepam was given for agitation and an esmolol drip was initiated. Labs were significant for potassium of 2.3 , carbon dioxide of 17 , and an anion gap acidosis with elevated lactate. The patient was admitted to the ICU for potassium repletion, continued symptomatic therapy, and telemetry monitoring.

Conclusion: Metabolic effects of caffeine overdose are successfully treated with beta-blockers and benzodiazepines. In our patient, treatment with magnesium and labetalol resolved the ventricular arrhythmia.

130. Laboratory Analysis of Ngirimbo Samples from Different Parts of Chitipa District: Attention on Toxicology

Vita Mithi ${ }^{1}$, John F. Kamanula ${ }^{2}$

${ }^{1}$ ARMREF Data for Action in Public Health Research, Mzuzu, North, Malawi. ${ }^{2}$ Department of Chemistry, Faculty of Science, Technology and Innovation, Mzuzu University, Luwinga, Mzuzu, North, Malawi

Background: Smokeless tobacco includes a wide variety of tobacco products which do not involve combustion and used orally or nasally. Ngirimbo is one of the oral smokeless tobacco substances used by smokers in Malawi as a tobacco harm reduction. The aim of the study was to determine the $\mathrm{pH}$, presence of volatile compounds, minerals (iron, zinc, calcium, magnesium, and copper) and heavy metals (lead, chromium, and cadmium) content in ngirimbo.

Methods: A well-established method that employs the use of atomic absorption spectrophotometer (AAS) and gas chromatography-mass spectrometry was adapted to estimate, nicotine content, concentration of toxic heavy metals, minerals of potential toxicity and other harmful chemicals in the different samples.

Results: Results show that the product contains harmful chemicals, high $\mathrm{pH}$ and nicotine [2-(1-methyl-2-pyrolidinyl)-pyridine, (S) - and (S)-3-(1methyl-2-pyrolidinyl)-pyridine] levels. The concentration of the analyzed minerals was found to be much higher than the required normal limits. On the other hand, the product does not hold lead, and it has low concentration of chromium and cadmium.

Conclusions: The results of this analysis suggest that prolonged use of ngirimbo product is a significant health risk to people with longlasting diseases. Nonetheless, the analysis points ngirimbo as component of tobacco harm reduction and a potential smoking cessation agent. Therefore, further toxicological studies of this nature will assist to establish and identify the nature of the product. 
131. Report of an 8-Year-Old Boy with Xylazine Complicating Fentanyl Poisoning, and Drug Detection Challenges

Emily L. Gill ${ }^{1,2}$, Ethan A. Mack ${ }^{1}$, Kevin C. Osterhoudt ${ }^{1,2}$, Leslie M. Shaw ${ }^{1}$, Michael C. Milone ${ }^{1}$

${ }^{1}$ University of Pennsylvania, Philadelphia, PA, USA. ${ }^{2}$ Children's Hospital of Philadelphia, Philadelphia, PA, USA

Background: The epidemic of opioid use disorder presents a major health challenge that worsened during the SARS-CoV2 pandemic. Children are becoming increasingly at risk of exposure to drugs of abuse, and pediatric emergency departments (PEDs) need to be aware of the toxidrome of an opioid/opiate and concurrent xylazine overdose, which is an increasingly common adulterant found in 'street heroin' across the USA.

Methods: This is a single patient case study. An eight-year-old male was found unresponsive and presented to a PED. The patient was hypopneic with respiratory acidosis treated with brief ventilation support and supplemental oxygen. Pinpoint pupils and hypotension were noted. The patient screened positive for SAR-CoV2 and was admitted to the special isolation unit (SIU). Drug screening was initially performed by immunoassay with secondary analysis by gas chromatography-mass spectrometry (GC-MS).

Results: Immunoassay drug screening was negative for opioids/opiates, oxycodone, and methadone; and co-oximetry excluded acute carbon monoxide poisoning. Within 24-hours, the boy returned to his baseline alertness, and was discharged with unclear etiology for the acute episode. However, 24-hours after discharge, GC-MS drug screening identified fentanyl, lidocaine and xylazine in the patient's urine, which are consistent for 'street heroin' with the clinical presentation.

Conclusion: Fentanyl is not found on many hospitals' immunoassay drug screening panels, and there is currently no commercially available immunoassay for xylazine. Pediatric hospitals should continuously evaluate their test menus for inclusion of emerging drugs like fentanyl. Xylazine has been rarely reported in drug analyses of children, but clinicians should be alert to its common presence; documentation of xylazine may identify the non-medical source of exposure. In patients with coingestion of xylazine and opioids/opiates, early detection of xylazine may guide therapy in the setting of naloxone failure.

132. "Haave"-ing a Severe Complication from Cannabinoid Hyperemesis: Esophageal Perforation in a Pediatric Patient

Sonya Rashid, Timothy Meehan

Toxikon Consortium, Chicago, IL, USA

Background: Cannabinoid hyperemesis syndrome is becoming more frequently seen in pediatric patients. The profound nausea and vomiting can have deleterious effects due to electrolyte abnormalities, hemorrhage, or structural injury. In adults, Boerhaave's syndrome (BS) has been rarely reported, but not in pediatric patients.

Methods: This is a single patient chart review. A 17-year-old female with a history of heavy daily marijuana use presented to the emergency room after a two-day duration of persistent nausea, vomiting, and diffuse abdominal pain. The patient had a similar episode of vomiting in March of 2021 and was diagnosed with urinary tract infection; she had no imaging at that time. In this presentation, the patient was given famotidine and three liters of fluids with no resolution of her symptoms, so a right upper quadrant ultrasound and $\mathrm{CT}$ abdomen and pelvis were ordered.

Results: CT A\&P was notable for pneumomediastinum. She was admitted to the PICU where a barium swallow test showed esophageal perforation. She was started on empiric antibiotics and antifungals. Nausea and vomiting were controlled with scheduled IV prochlorperazine and topical capsaicin cream, with haloperidol as needed for breakthrough symptoms. The patient was initially on total parenteral nutrition, then gradually to liquid diet and soft foods. Pediatric gastroenterology and surgery followed the patient. No surgical intervention took place. Pneumomediastinum showed gradual improvement with daily chest $\mathrm{x}$-ray. The patient was discharged on hospital day 11 and has been doing well in follow up without complication.

Conclusion: Cannabinoid hyperemesis syndrome is an emerging problem in pediatric patients and can cause BS; this is the first reported case in the literature.

133. Falsely Elevated Serum Ethanol in an Infant's Peri-Mortem, Post Cardiac Arrest, Toxicology Testing

Zachary Miller ${ }^{1}$, Beverly Anderson ${ }^{2}$, Colleen Bennett ${ }^{1}$, Derick Lim ${ }^{1}$, Kevin Osterhoudt ${ }^{1}$

${ }^{1}$ Children's Hospital of Philadelphia, Philadelphia, Pennsylvania, USA.

${ }^{2}$ Nemours Children's Hospital, Wilmington, Delaware, USA

Background: Children presenting for emergent care commonly undergo screening for toxic exposures, which are potentially reversible causes of emergent scenarios including cardiorespiratory arrest, acutely altered mental status, seizures, and cardiac arrhythmias. While no toxicologic testing guidelines apply in all cases, screening often includes ethanol.

Methods: This is a single patient chart review. A four-month-old female was found unresponsive at home. She received over 60 minutes of resuscitation before return of spontaneous circulation. Her evaluation showed inotrope-refractory shock, anemia, pulmonary hemorrhage, hyperlactatemia with acidosis, elevated serum transaminases, elevated PT/PTT, and acute kidney injury, indicating multisystem organ failure. She had no purposeful movements, and her EEG was featureless. Life support was withdrawn, and the patient died one day after presentation. Ethanol testing by enzymatic assay, obtained 14.5, 18.5, and 23 hours after presentation, showed ethanol levels of $13 \mathrm{mg} / \mathrm{dL}, 14 \mathrm{mg} / \mathrm{dL}$, and $15 \mathrm{mg} / \mathrm{dL}$ (reference range $<10 \mathrm{mg} / \mathrm{dL}$ ), respectively. Plasma lactate, measured simultaneously with serum ethanol, ranged from $16 \mathrm{mmol} / \mathrm{L}$ to $>25 \mathrm{mmol} / \mathrm{L}$ (reference range $0.5-1.6 \mathrm{mmol} / \mathrm{L}$ ). Serum LDH was $>16,000 \mathrm{U} / \mathrm{L}$ (reference range 470-1220 U/L). Confirmatory GCMS testing of the sample with the highest ethanol level was negative.

Discussion: Extreme elevations of lactate and LDH may interfere with enzymatic ethanol assays via the NAD+/NADH system, a phenomenon that may be unfamiliar to clinicians and that has important implications for interpreting a common drug screen. Laboratory analysis of this case supports such interference creating a false positive in our patient. This is one of few case reports showing a false-positive ethanol level in a pediatric patient under similar circumstances; most cases are taken from postmortem samples, in contrast to our patient.

Conclusion: Toxicologic workup is common for infants presenting in extremis secondary to unexplained cardiac arrest. Unexpected positive ethanol levels should be evaluated accordingly for possible interference due to elevated lactate and LDH.

134. Continuous Glucose Monitoring Versus Point-of-Care Glucose Testing for a Pediatric Calcium Channel Blocker Overdose Rebecca Raffler ${ }^{1}$, Lauren Piper ${ }^{1}$, Ann-Jeannette Geib ${ }^{2}$, Christine Murphy $^{2}$

${ }^{I}$ Atrium Health's Levine Children's Hospital, Charlotte, NC, USA. ${ }^{2}$ Atrium Health's Carolinas Medical Center, Charlotte, NC, USA

Background: Patients admitted for calcium channel blocker (CCB) overdoses require frequent glucose checks during high dose insulin (HDI) therapy. Continuous glucose monitoring (CGM) is cost-effective, less invasive, and more efficient in comparison to POC glucose monitoring (PGM).

Research Question: Will CGM be more cost-effective in comparison to PGM in this patient population?

Methods: This is a case report of a teenager with vasoplegic and cardiogenic shock secondary to CCB overdose and asymptomatic COVID-19 infection. He required frequent glucose checks while on HDI therapy (20 units $/ \mathrm{kg} /$ hour for three days before weaning) and dextrose infusion ( 8.5 
days). Pricing data was collected using Healthcare Bluebook, discussion with diabetes educators, and CGM representatives. Nursing input obtained on efficiency for CGM in comparison to PGM.

Results: The patient's glucose was checked 245 times while on HDI therapy and D50 infusion. PGM was utilized for the first 26 measurements followed by CGM. PGM supplies include: monitor (\$33.90), reagent strips (\$0.18/ea), and lancets (\$0.39/ea). CGM supplies include a reader (\$63) and one transmitter/10 days (\$59). The ICU owned the CGM reader and POC glucose monitor. When compared, the cost of supplies to run all 245 glucose checks would have been $\$ 139.65$ with PGM and \$59 for CGM.

Conclusion: HDI therapy is an effective and life-saving treatment in the management of moderate-to-severe toxicity due to CCB overdose. It requires ongoing monitoring of organ perfusion, cardiac function, glucose, and potassium. Our patient had 245 glucose checks in his 8.5-day course of D50 infusion. CGM is more cost-effective and time-efficient by reducing the number of nursing tasks and finger sticks in comparison to PGM. These outcomes should prompt providers to consider CGM for use in this patient population.

\section{A 15-fold Zoledronic Acid Overdose in a Pediatric Patient with} Osteogenesis Imperfecta

Mirjana Dimovska ${ }^{1}$, Varun Vohra $^{1}$, Lydia L Baltarowich ${ }^{2}$, Andrew M King $^{1}$, Bram A Dolcourt ${ }^{1}$

${ }^{1}$ Wayne State University, Detroit, Michigan, USA. ${ }^{2}$ Henry Ford Hospital, Detroit, Michigan, USA

Background: Zoledronic acid (ZA), a potent intravenous (IV) bisphosphonate commonly used for fracture prevention in osteoporosis and hypercalcemia in malignancy, is used off-label in children with osteogenesis imperfecta (OI). Little data exists on overdose.

Methods: Two year-old, $10.8 \mathrm{~kg}$ male with OI was admitted to the ICU after $4 \mathrm{mg}(0.37 \mathrm{mg} / \mathrm{kg})$ ZA was administered instead of $0.27 \mathrm{mg}(0.025$ $\mathrm{mg} / \mathrm{kg}$ ). Calcium and phosphorus were supplemented following declining serum levels. Ergocalciferol was administered daily. On Hospital Day (HD) four, hemodialysis was initiated for electrolyte correction and potential removal of ZA. On HD7, multiple runs of nonsustained ventricular tachycardia were observed. Calcium and phosphorus dropped to 6.4 and $1.7 \mathrm{mg} / \mathrm{dL}$, respectively. Albumin and creatinine remained relatively unchanged. The child was discharged on HD11.

Discussion: ZA is a rare overdose. Typical pediatric off-label dosing is $0.05 \mathrm{mg} / \mathrm{kg}$. This child received a near 15 -fold overdose. In therapeutic doses, hypocalcemia is reported in $7-12 \%$ of pediatric patients, with severe hypocalcemia in $\sim 3 \%$, within three days post-infusion. In a study of fixed dose $5 \mathrm{mg}$ ZA infusion in children ages 2-16 with OI, 100\% (18) developed hypophosphatemia and $86 \%$ (15) developed hypocalcemia. No electrolyte-related adverse outcomes were reported. The timeframe and duration were not reported.

ZA has triphasic elimination with a half-life of $0.23,1.75$, and 167 hours, respectively. Approximately $39-46 \%$ is recovered from the urine in the first 24 hours. Intermittent hemodialysis corrects electrolyte abnormalities but is unlikely to eliminate clinically significant amounts of ZA. Due to ZA's long elimination half-life, prolonged monitoring may be warranted. Conclusion: Pediatric ZA overdose may cause significant hypocalcemia and hypophosphatemia. Asymptomatic, non-sustained ventricular tachycardia was observed in our patient. Protracted inpatient and outpatient monitoring with electrolyte supplementation is a reasonable treatment approach

136. Auto-brewery Syndrome in a 23-Month-Old Presenting as Hypoglycemia and Altered Mental Status

Thao-phuong C Hallett ${ }^{1,2}$, Zachary N Illg ${ }^{1,2}$, Nicholas V Titelbaum ${ }^{1,2}$, Johnathan D de Olano ${ }^{1,2}$

${ }^{1}$ Emory University School of Medicine, Atlanta, GA, USA. ${ }^{2}$ Georgia Poison Center, Atlanta, GA, USA
Background: Auto-brewery syndrome is a rare condition in which the gut flora produces endogenous ethanol within the gastrointestinal tract. Cases have been described in children with short bowel syndrome and altered gut flora but not in a healthy child.

Hypothesis: Fermenting bacteria in the alimentary tract is a recurring source of endogenous ethanol, causing hypoglycemia and altered mental status.

Methods: This is a case report. A 23-month-old toddler with no significant past medical history presented to a pediatric emergency department (PED) with altered mental status and hypoglycemia. A diarrheal episode with somnolence was reported earlier in the day. Prehospital glucose was $50 \mathrm{mg} / \mathrm{dL}$, so an oral glucose load was given. Initial GCS was nine (E2V3M4) with pinpoint pupils and otherwise normal neurologic exam. Initial PED workup revealed glucose was $88 \mathrm{mg} / \mathrm{dL}$, mild metabolic acidosis with a $\mathrm{pH}$ of 7.25 , bicarbonate level was $17 \mathrm{mg} / \mathrm{dL}$, positive ketones on urinalysis, and ethanol level of $13 \mathrm{mg} / \mathrm{dL}$. The patient's mother denied accessible ethanol-containing products at home. After returning to baseline mental status, a PO challenge with a popsicle was given, and he became altered again.

Results: The patient was somnolent but arousable and admitted for observation. A repeat ethanol level was $19 \mathrm{mg} / \mathrm{dL}$. On hospital day two, an ethanol level was undetectable. Oatmeal was given for breakfast, and the patient became somnolent. Repeat post-prandial ethanol level was $16 \mathrm{mg}$ $\mathrm{dL}$ four hours after breakfast. Subsequent increases of serum ethanol with carbohydrate loads were consistent with auto-brewery syndrome. A ketogenic diet and fluconazole were started, and the patient was discharged with continued outpatient monitoring for recurrence to date.

Conclusion: Auto-brewery syndrome should be considered in the differential diagnosis for a child without a history of ethanol exposure but a detectable ethanol level even in the absence of short gut syndrome.

137. Intravenous Lipid Emulsion for Ivermectin Toxicity in an EightYear-Old Male

Molly K Stott ${ }^{1,2}$, Martha S Phillips ${ }^{3,4}$, Sophia Sheikh ${ }^{5,6}$

${ }^{1}$ UF Health Jacksonville, Jacksonville, FL, USA. ${ }^{2}$ Florida/USVI Poison Information Center - Jacksonville, Jacksonville, FL, USA. ${ }^{3}$ Baptist Medical Center, Jacksonville, FL, USA. ${ }^{4}$ Wolfson Children's Hospital, Jacksonville, FL, USA. ${ }^{5}$ University of Florida College of Medicine Jacksonville, Jacksonville, FL, USA. ${ }^{6}$ Florida/USVI Poison Information Center- Jacksonville, Jacksonville, FL, USA

Background: Preliminary trials showing possible benefit of ivermectin treatment for COVID-19 infections has led to an unprecedented awareness of the drug by the public. This sparked an increase in the public seeking ways to obtain and consume ivermectin for COVID-19 and other ailments. While the treatment of ivermectin toxicity using intravenous lipid emulsion (ILE) in animals has been studied, there is little evidence demonstrating its benefit in cases of human toxicity.

Hypothesis: Is ILE safe and effective in the treatment of ivermectin toxicity?

Methods: This is a case report of an eight-year-old male given veterinary grade ivermectin ointment bought from a feed store to treat a presumed parasitic infection on his foot. Ivermectin was given orally by his grandmother after reading information from the internet regarding treatment options for parasitic infections. The child was given six grams (250 $\mathrm{mg} / \mathrm{kg}$ ) of ivermectin ointment. Three hours post-administration of ivermectin the child was found unresponsive and was brought to the emergency department where he developed multiple seizures refractory to benzodiazepines. The poison control center was consulted for recommendations regarding use of ILE. The medical team elected to administer ILE given the patient's declining status. The patient received a $1.5 \mathrm{~mL} / \mathrm{kg}$ IV bolus over three minutes $(31 \mathrm{mg})$, then began the infusion at $0.025 \mathrm{~mL} /$ $\mathrm{kg} /$ minute over six hours (41.4 g).

Results: The patient received a total of $72.4 \mathrm{~g}$ of ILE over six hours while triglycerides were monitored closely. Three hours after the infusion began the patient's mental status returned to baseline. There was no recurrence 
of seizure activity or adverse effects from the ILE. The etiology of the patient's foot rash was never determined.

Conclusion: ILE therapy may be a potential treatment option in cases of severe ivermectin overdose that is refractory to other supportive measures.

138. Do Not Eat! A Case of Iron Toxicity from Beef Jerky Oxygen Absorber Desiccant Packet

Kai J Norbeck, Adam M Spanier, Andrew D Zabel, Alexander F Barbuto

Carl R. Darnall Army Medical Center, Fort Hood, Texas, USA

Background: Iron toxicity was historically a leading cause of pediatric poisoning deaths and requires early identification and diagnosis. Iron toxicity from prenatal vitamins or supplements is well described in the literature. We present a case of iron overdose from an oxygen absorber packet, an infrequently encountered source of potential iron toxicity, illustrating that not all preservative packets are mere non-toxic choking hazards.

Methods: This is a single patient case report. A five-year-old presented to the Emergency Department (ED) after ingesting the contents of an oxygen absorber packet from beef jerky packaging. As the patient's parents assumed the packet was a silica desiccant, local poison control was initially unconcerned. However, it later became apparent that this was an iron-based oxygen absorber, and the patient proceeded to the ED.

Results: Oxygen absorbing packets can contain approximately 1-15 grams of elemental iron. In the ED, the patient was asymptomatic with normal vital signs. Abdominal $\mathrm{X}$-rays did not reveal any radiopaque objects. Initial iron level was $248 \mathrm{mcg} / \mathrm{dL}$ two and a half hours after ingestion. Iron levels peaked at $322 \mathrm{mcg} / \mathrm{dL}$ six hours after ingestion, then decreased to $219 \mathrm{mcg} / \mathrm{dL}$ at nine hours post-ingestion. Three days later, the iron level normalized to $110 \mathrm{mcg} / \mathrm{dL}$.

His iron peaked at moderate systemic toxicity range. As the patient remained asymptomatic, normotensive, without signs of end organ damage, treatment was not initiated, and he was discharged home once iron levels decreased.

Conclusions: Oxygen absorber packets resemble non-toxic silica desiccants but have potential devastating consequences if missed. They are ubiquitous and are present in various food packaging. Recognizing that not all preservative packets are non-toxic is crucial in identifying a potentially significant poisoning. Although our patient did well, a younger child may suffer significant iron overdose from these oxygen absorber packets.

139. A Five-Year Review of Vanilla Extract Exposures Reported to the California Poison Control System

Emmanuel A Quaidoo ${ }^{1,2}$, Jacob A Lebin ${ }^{1,2}$, Anita K Mudan ${ }^{1,2}$, Daniel J Repplinger $^{1,2}$

${ }^{I}$ Department of Emergency Medicine, University of California San Francisco, San Francisco, California, USA. ${ }^{2}$ California Poison Control System, San Francisco, California, USA

Background: Vanilla extract is a common household product containing hundreds of natural compounds, namely vanillin, steeped in ethanol. In addition to ethanol intoxication, lay reports have suggested that these naturally occurring compounds possess psychoactive properties with large ingestions. However, vanilla extract exposures have yet to be well described in the literature. We sought to characterize vanilla extract exposures reported to the California Poison Control System (CPCS).

Methods: We performed a five-year retrospective review of all vanilla extract exposures, with the keyword vanilla, reported to the CPCS from January 2015 through December 2019. Patient characteristics, including demographics and ingestion type, as well as clinical course (symptoms, treatment, and outcomes) were extracted from the CPCS database.

Results: A total of 110 vanilla extract cases were identified. The majority of cases were unintentional $(\mathrm{n}=73,66 \%)$ and occurred in children $(\mathrm{n}=$ $70,96 \%$ ). Of unintentional exposures, the majority were asymptomatic (n
$=62,85 \%)$ and managed at home $(n=70,96 \%)$. Conversely, the majority of intentional exposures were in adults $(\mathrm{n}=28,90 \%)$, and presented or were referred to a hospital for evaluation $(\mathrm{n}=27,77 \%)$. Few vanilla extract exposures were coded as self-harm attempts $(n=6,5 \%)$, but half of these were in children and all presented to the hospital. Nonspecific GI symptoms $(\mathrm{n}=8,27 \%)$, CNS depression $(\mathrm{n}=19,63 \%)$, and tachycardia ( $\mathrm{n}=8,27 \%$ ) were the most common symptoms in symptomatic exposures. Three cases developed respiratory depression which improved with supplemental oxygen. One self-harm attempt was admitted to the ICU for observation. No major effects or deaths were identified. Intentional exposures, on average, had larger vanilla extract ingestions than unintentional exposures ( 577 vs. $29 \mathrm{~mL}$ ).

Conclusions: Most vanilla extract exposures occurred in children and were unintentional and asymptomatic. GI symptoms, CNS depression, and tachycardia were common following symptomatic exposures, but there was no report of psychoactive features in this cohort.

\section{Board Certification Trends of Medical Toxicologists in Addiction} Medicine

Sefira B Fialkoff ${ }^{1}$, Paul Wax ${ }^{1}$, Timothy J Wiegand ${ }^{2}$

${ }^{1}$ American College of Medical Toxicology, Phoenix, AZ, USA.

${ }^{2}$ University of Rochester Medical Center, Rochester, NY, USA

Background: Addiction medicine (AM) encompasses substantial core content that overlaps with medical toxicology. In addition, AM may offer additional practice and professional opportunities beyond standard medical toxicology practice. In 2017, the American Board of Preventive Medicine (ABPM) began to offer a subspecialty certificate in Addiction Medicine. Previously, such board certification (BC) in AM was offered by the American Board of Addiction Medicine (ABAM).

Research Question: How many board-certified medical toxicologists are also board certified in addiction medicine?

Methods: Two thousand and twenty one data from the American Board of Medical Specialties on all board-certified medical toxicologists (MT) including those who were also board certified in AM from ABPM through the 2020 examination cycle was obtained. Data was also obtained from ABAM on all board-certified MT also board certified by ABAM through 2016. This data was used to determine the number of boardcertified MT that were board-certified in AM by ABPM and/or ABAM, primary BC, and percent of dual board-certified MT and AM.

Results: Eighty board certified MT were dual board certified in addiction medicine by ABPM and/or ABAM, as of 2021. Five of 80 were ABAM certified only, 28 were ABAM certified and then became ABPM certified, 47 became certified by ABPM. Of these $80,67(84 \%)$ have primary boards in emergency medicine. As of 2021 there are 555 ABMS board certified MT with active certificates. The 75 with ABPM AM certification represent $14 \%$ of all MT. Conclusion: Fourteen percent of boardcertified MT are also board certified in AM. Further study of the impact of this additional board certification is warranted.

141. Rapid Dissemination of Timely COVID-19 Information Through a Weekly Webinar Series

Mukta N Deia ${ }^{1}$, Paul Wax ${ }^{1}$, Ziad Kazzi ${ }^{2}$, Charles McKay ${ }^{3}$, Adrienne Dunavin $^{1}$, Jessica Irving ${ }^{1}$

${ }^{1}$ American College of Medical Toxicology, Phoenix, AZ, USA. ${ }^{2}$ Emory University School of Medicine, Atlanta, GA, USA. ${ }^{3}$ University of Connecticut School of Medicine, Hartford, CT, USA

Background: At the outset of the COVID-19 pandemic in March 2020, hydroxychloroquine (HC) was touted as a therapeutic in treating the novel SARS-CoV-2 virus infection. With rising concern about $\mathrm{HC}$ toxicity, ACMT presented a webinar about the potential adverse effects of HC. When over 500 participants attended this webinar, ACMT recognized the thirst for information about COVID-19 and initiated a weekly webinar series, entitled "Medical and Public Health Considerations of COVID-19." 
Research Question: Can a small-sized medical society disseminate timely information to a vast audience of healthcare professionals during a pandemic?

Methods: ACMT formed a task force of its members and staff to lead its COVID-19 response and generate timely webinars (and related resources). Leading experts from across the globe were recruited to present on topics, including (but not limited to) clinical manifestations, testing, treatments, vaccines, and public health response. ACMT reviewed speakers' content before the webinars, which were hosted via the webinar platform (WebEx and Zoom). Partnerships emerged with ten domestic and international medical societies to disseminate information about the webinar series. Two grants were obtained to support this effort. Data was collected on all webinar attendees, including their location and occupation type.

Results: ACMT produced 49 webinars and identified 69 plenary speakers to provide expertise on various COVID-19 topics. 67,652 people registered for the webinars. $50.2 \%$ of registrants attended, with an average of 743 per webinar. Thirty-two medical and public health profession categories were represented among attendees (on average: $30 \%$ nurses, $20 \%$ physicians, $10 \%$ public health professionals, $5 \%$ pharmacists and others in several specialties). The webinar series attracted attendees from 82 countries and was recorded for on-demand viewing, which amassed over 28,000 views.

Conclusion: A weekly webinar series organized by a small medical organization can effectively disperse timely COVID-19 information to reach a wide range of healthcare professionals globally.

142. National Antidote Network in Taiwan, 2001-2020

Hsiang-Ling Chen ${ }^{1}$, Jou-Fang Deng ${ }^{1}$, Ming-Ling Wu ${ }^{1}$, Chen-Chang Yang ${ }^{1,2}$

${ }^{1}$ National Poison Control Center \& Division of Clinical Toxicology \& Occupational Medicine, Department of Medicine, Taipei Veterans General Hospital, Taipei, Taiwan. ${ }^{2}$ Institute of Environmental \& Occupational Health Sciences, School of Medicine, National Yang Ming Chiao Tung University, Taipei, Taiwan

Background: Except in a few technologically advanced countries, the lack of antidote has been a universal issue. For chemical disaster preparedness, in Taiwan, a national antidote network was established by the Poison Control Center in 2000. Starting with selection and procurement of antidotes followed by distribution and feedback of antidotes use was the mainframe operation of our antidotes supply network. This study was conducted to better understand the performance of the national antidote network after its full operation in 2001.

Methods: Feedback of antidotes use included patient information, exposed substance, diagnosis, using the dosage of antidote, and treatment outcome. Data were retrieved from all patients treated with antidotes between 2001 and 2020.

Results: Cyanide poisoning antidotes (Cyanide Kit and cyanocobalamin), physostigmine, digoxin immune fab (DigiFab), heavy metal chelating agents (Ca-EDTA/DMSA/DMPS), fomepizole, and methylene blue were the antidotes made available by Taiwan PCC through the procedure of international procurement. From 2001 through 2020, there were 666 patients who received antidote treatment provided by the national antidote network. Among them, 112 cases received cyanide antidotes treatment with clinical improvement being observed in $43 \%$ of cases. By contrast, 238 received physostigmine, 68 received DigiFab, 145 received heavy metal chelators, 15 received fomepizole, and 86 received methylene blue, with a clinical improvement being observed in $87 \%, 56 \%, 71 \%$, and $33 \%$ of treated patients, respectively.

Conclusion: Some antidotes are essential in the treatment of specific poisonings, and they could be lifesaving. However, most of the antidotes do not carry commercial incentive, therefore special efforts to make the antidotes available for clinical emergency is warranted for exploration. The operation experience of Taiwan's national antidote network may be worthwhile for international reference.

143. Medical Toxicology Mini-Fellowship: An Extended Toxicology Curriculum in an Emergency Medicine Residency

Noah G Berland ${ }^{1,2}$, Trevor Cerbini ${ }^{2,3}$, Paul N Hein ${ }^{2,3}$, Adam D Blumenberg ${ }^{4}$, James Willis ${ }^{3,2}$, Sage Wiener ${ }^{2,3}$

${ }^{1}$ Rutgers NJMS, Newark, NJ, USA. ${ }^{2}$ Kings County Hospital, Brooklyn, NY, USA. ${ }^{3}$ SUNY Downstate, Brooklyn, NY, USA. ${ }^{4}$ Columbia University, New York, NY, USA

Background: Medical Toxicology represents four percent of questions on the Emergency Medicine (EM) qualifying exam. However, medical toxicology is a challenging and expansive field with a low frequency of critical cases at any given institution. Our program is a four-year Emergency Medicine residency in an urban tertiary level one trauma center. We developed a dedicated mini fellowship to supplement toxicology education for self-selected EM residents. Methods: We reviewed and categorized four years (July 2017 - June 2021) of mini-fellowship meetings and characterized the topic areas covered. All meetings were supervised and reviewed by a board-certified Medical Toxicologist and EM physician and were presented by a self-selected resident. Asynchronous learning credit was provided to all residents attending the session.

Results: We averaged five mini-fellowship meetings a year, with a standard deviation of 2.55 , which was likely reduced by the COVID-19 pandemic, as the academic year of 2019-2020 only had two meetings. A number of meetings were held jointly with other mini fellowships, including Critical Care, Emergency Medical Services, Disaster, Geriatrics, Education, and Wilderness. Residents selected the following topics for discussion: organophosphates, cyanide, opiates, naloxone, pseudoscientific remedies, drug adulteration, nicotine, digoxin, lithium, asphyxiates, thallium, buprenorphine, general approach to a poisoned patient, methyl mercury, licorice, hydroxychloroquine, radiation, mushrooms, and geriatric toxicology.

Discussion: Our toxicology mini fellowship allowed residents to explore their own interests and develop experience teaching toxicology topics. The selected topics may indicate gaps in traditional EM curricula as they were chosen by residents for supplementary study.

Conclusion: A toxicology mini fellowship supplemented toxicology education in our EM residency.

144. ToxRunner: Creation and Distribution of a Free Medical Toxicology Practice Question Mobile App for iOS and Android

Adam Blumenberg

Columbia University Medical Center, New York City, NY, USA

Background: Digital practice questions are an effective means of learning medical knowledge. The mobile application "ToxRunner" was created to meet the need for a mobile toxicology question application.

Methods: A needs assessment was performed by reviewing the toxicology core curriculum, researching practice question pedagogy, reviewing available study materials, and by interviewing fellows, toxicologists, and toxicology program directors. The results of the needs assessment indicated that an easily accessible mobile app with high quality questions was needed to augment toxicology education. A software platform named "ToxRunner" was created using Microsoft Visual Studio operating Xamarin. The software utilized XAML for the user interface, C\# for the code-behind, JSON objects for data manipulation, and MySQL for internet database functionality. The software allows for user profiles, graphical exam score trending, exam review, timed/untimed/review modes, and question feedback. Based on medical didactics guidelines, 390 questions were written and entered. The application was distributed gratis on the iOS and Android app-stores.

Results: Within six months, 117 users registered, and 91 learners attempted one or more practice questions. Twenty-six individuals registered the application but completed zero questions; these were excluded 
from analysis. The 91 active learners were 47 toxicology fellows (52\%), 41 toxicology attendings (45\%), two pharmacists (2\%), and one resident (1\%). Each of the 91 learners completed an average of 421.7 practice questions with a median of 380 , a standard deviation of 384.9 , and a maximum of 1924 . Sixty-six learners $(73 \%)$ completed 100 or more questions.

Discussion: The average learner not only completed all 390 available questions, but also repeated questions. The large average amount of time spent using ToxRunner implies that learners valued ToxRunner as an educational resource.

Conclusion: ToxRunner is a free mobile practice question application for toxicology education. Learners who tried the application went on to complete hundreds of practice questions.

145. Measuring the Effectiveness of Sniff Bars in Recognition of Medically Important Odors in Graduate Medical Education

Shaza Aouthmany ${ }^{1}$, Mitchell Pei ${ }^{1}$, Morgan Bailey ${ }^{1}$, Sameer Syed ${ }^{1}$, Ola $\mathrm{Kebbi}^{2}$, Luke Bisoski ${ }^{1}$

${ }^{1}$ University of Toledo, Toledo, OH, USA. ${ }^{2}$ American University of Beirut, Beirut, Lebanon

Background: To increase the recognition of odors connected to toxic products, Dr. Goldfrank et al. prepared a "sniffing-bar" composed of odors resembling toxic substances or chemicals and paired these scents with associated case studies. It was determined that the "sniffing-bar" could potentially supplement training to better prepare clinicians to detect odors that may aid in diagnosis or treatment.

Methods: This is a voluntary sample survey given in January 2021 at the University of Toledo to determine residents' confidence in identifying medically important odors after using the sniff bar. Confidence was assessed using a visual analog scale before and after a sniff bar simulation in which subjects were asked to smell and identify nine distinct odors paired with detailed case studies. Data analysis was performed using paired T-tests at a $95 \% \mathrm{CI}$.

Results: There were 18 individuals who met inclusion criteria. There was a significant increase in the confidence of residents to identify medically important odors after an education session with a sniff bar (95\% CI 1.31 to 2.25). In addition, when asked if the participants felt confident in responding to a medically important odor with the proper steps and techniques, there was a significant increase in mean confidence (95\% CI 1.33 to 2.45). The study also showed that following the sniff bar simulation, residents felt more confident being the team lead during a medically important odor scenario, were satisfied with their ability to handle the patient, and felt confident in performing a code related to a medically important order in a calm manner.

Conclusion: Using a sniff bar during simulation on toxicology education was effective in increasing residents' ability to recognize and treat toxic odors of rare clinical scenarios. The sniff bar should be used as a supplemental educational tool to help residents recognize toxic odors earlier in their training.

146. Methemoglobinemia and Angioedema After Topical Exposure to para-Phenylenediamine (PPD)

Matthew S Correia ${ }^{1,2}$, B Zane Horowitz ${ }^{1,2}$, Matthew Noble ${ }^{1,2}$

${ }^{I}$ Oregon Health and Science University, Portland, OR, USA. ${ }^{2}$ Oregon

Poison Center, Portland, OR, USA

Background: Para-phenylenediamine (PPD) is a common component of dark-colored hair dye products and occasionally black henna ink. Upwards of six percent of individuals in North America display contact sensitivity to PPD. It is readily absorbed by all routes of exposure and exerts toxicity via its irritating and oxidizing effects. Systemic toxicity usually occurs only after oral ingestion and is characterized by angioedema, methemoglobinemia, and hemolysis.

Methods: A 65-year-old female with cerebral palsy presented to the emergency department with facial swelling and painful scalp erythema after topical exposure to a darkening hair dye product two-day prior. She was well appeared with a pulse of 136 beats per minute, blood pressure $126 / 67 \mathrm{mmHg}$, respiratory rate 28 breaths per minute, oxygen saturation $95 \%$ on one-liter nasal cannula, and afebrile. Her scalp was markedly erythematous, crusted, and weeping. She exhibited significant periorbital swelling and facial edema. She had no dysphonia and her oropharynx and larynx were normal. There was no cyanosis. Blood work revealed: $\mathrm{pH}$ 7.39, $\mathrm{pCO}_{2} 42 \mathrm{mmHg}$, methemoglobin $15.2 \%, \mathrm{HCO}_{3} 24 \mathrm{mg} / \mathrm{dL}$, Creatinine $0.74 \mathrm{mg} / \mathrm{dL}, \mathrm{CK} 43 \mathrm{IU} / \mathrm{L}$, and hemoglobin $15.4 \mathrm{mg} / \mathrm{dL}$. Her scalp was cleansed. She was admitted to the hospital for observation and symptomatic relief. The methemoglobinemia gradually resolved without further intervention.

Discussion: Prior reports of methemoglobinemia in the setting of paraphenylenediamine are restricted to oral exposures. This patient did not develop hemolysis, renal failure nor life-threatening angioedema. However, misapplication, or inadequate post-application care has the potential to cause serious harm, as significant dermal absorption may occur in the setting of prolonged exposure. Due to the risk of adverse effects and contact allergy, the Federal Drug Administration previously banned its use in henna products in the United States.

Conclusion: This case, along with other similar reports, should warrant reconsideration of the safety of PPD as a cosmetic hair dye additive.

147. Voltammetric Approach of Arsenic (Total) Determination in Urine using Sc Trace Gold Electrode

Ashok Kumar Jaiswal, Millo Tabin, Sudhir Kumar Gupta

All India Institute of Medical Sciences, Delhi, India

Background: Urine is suitable biological material to check for acute arsenic poisoning. Normal level for arsenic in urine is less than $100 \mathrm{ppb}$. Various sophisticated analytical techniques like ICP-MS, AAS etc. are usually used to assess levels of arsenic in urine. An attempt has been made to develop the method for determination of arsenic (total) in urine with voltammetric technique using Sc Trace gold electrode.

Hypothesis: Sc Trace gold electrode can be used for determination of arsenic (total) in urine.

Methods: This is an experimental study. Urine was mixed with $\mathrm{HNO}_{3}$ and digested in a microwave digestor. Quantitative determination of arsenic (total) was done using a voltammetric approach with Sc Trace Gold Electrode. The working electrode is a gold micro wire whereas reference and auxiliary electrodes are screen printed electrodes. Arsenic (total) determination was carried out by standard addition technique using primary solution. As (V) species being electrochemically inactive are reduced in-situ by nascent hydrogen to As (III). Along with the As (III) present in the sample, it is further reduced electrochemically to As (0) and deposited on the gold working electrode. The deposited metal was swept by scanning the potential from $-0.300 \mathrm{~V}$ to 0.400 using DC wave mode. The stripping current was correlated with the concentration of the metal in the urine sample. The detection limit of arsenic (total) by this method is found to be $1.0 \mu \mathrm{g} / \mathrm{L}$ and linear up to a concentration of $20 \mu \mathrm{g} / \mathrm{L}$.

Results: Arsenic (total) in urine was successfully quantified at very low levels using Sc Trace Gold Electrode.

Conclusion: Developed method is very sensitive, reproducible and inexpensive which can be used to determine arsenic (total) level in urine in a very simple way. 
2022 ACMT Annual Scientific Meeting Abstracts - Virtual

\begin{tabular}{|c|c|}
\hline Author name & Abstract No. \\
\hline Al Bulushi, Hassan & 045 \\
\hline Alsulaimani, Suad & 045 \\
\hline Al Sukaiti, Waleed & 045 \\
\hline Abston, Stephanie & $022,040,109,112$ \\
\hline Acciani, Jennifer & 068,084 \\
\hline Adkins, Alek & 076,125 \\
\hline Adnan, Syed & 026 \\
\hline Afaria, Neha & 035,061 \\
\hline Albertson, Timothy & 019,085 \\
\hline Aldy, Kim & $\begin{array}{l}004,013,022,038,040,074, \\
\quad 105 \\
109,112,114,115,117,118, \\
\quad 138,143\end{array}$ \\
\hline Alizadeh, Anahita & 004 \\
\hline Allen, Katherine & 050 \\
\hline Amaducci, Alexandra & $040,047,079,101$ \\
\hline Ancona, Rachel & 010 \\
\hline Anderson, Beverly & 133 \\
\hline Aouthmany, Shaza & 145 \\
\hline Appelbaum, David & 113 \\
\hline Arshad, Faizan & 042 \\
\hline Arslan, Aleyna & 033 \\
\hline Atti, Sukhshant & 074 \\
\hline Avakian, Tayler & 085 \\
\hline Avera, Robert & 058 \\
\hline Avula, Bharathi & 011 \\
\hline Baig, Muhammad Akbar & $\begin{array}{l}\text { 046, 048, 051, 066, 071, 073, } \\
\quad 097\end{array}$ \\
\hline Bailey, Morgan & 145 \\
\hline Bakkar, Azza & 060 \\
\hline Baltarowich, Lydia & 135 \\
\hline Barbuto, Alexander & $045,129,138$ \\
\hline Battaglia, Christopher & 151 \\
\hline Baumgartner, Kevin & 067,084 \\
\hline Becker, Sarah & 055 \\
\hline Bell, Belinda & 005 \\
\hline Bello, Miriam & 042 \\
\hline Beltran, Mayra & 094 \\
\hline Benavente, Susana & 087 \\
\hline Bennett, Colleen & 133 \\
\hline Berg, Sarah & 159 \\
\hline Bergeron, Bryce & 195 \\
\hline Berland, Noah & $043,080,143$ \\
\hline Berman, Adam & 046 \\
\hline Beuhler, Michael & 074 \\
\hline Bhagat, Sunita & 035 \\
\hline Bhalla, Ashish & 093 \\
\hline Bisoski, Luke & 145 \\
\hline
\end{tabular}

\begin{tabular}{|c|c|}
\hline Bloom, Joshua & 107 \\
\hline Blumenberg, Adam & 143,144 \\
\hline Borek, Heather & 070 \\
\hline Boussi, Leora & 060 \\
\hline Boyle, Katherine & 060 \\
\hline Brambl, Wells & 066,088 \\
\hline Branch, Hilary & 081 \\
\hline Brandehoff, Nicklaus & 050 \\
\hline Bremer, Whitney & 116 \\
\hline Brenner, Marielle & $058,068,107$ \\
\hline Brent, Jeffrey & $\begin{array}{l}004,013,022,038,040,074, \\
\quad 109,112, \\
114,115,117,118,138\end{array}$ \\
\hline Brewer, Kori & 037,125 \\
\hline Brooks, Daniel & 077 \\
\hline Brooks-Russell, Ashley & 002 \\
\hline Buchanan, Jennie & 050 \\
\hline Buckley, Nicholas & 001 \\
\hline Burack, Seth & 106 \\
\hline Burkhart, Keith & 013 \\
\hline Burns, Michele & $055,065,072,081,113$ \\
\hline Burris, Olivia & 099,100 \\
\hline Calello, Diane & $043,080,115$ \\
\hline Calleo, Vincent & 026,028 \\
\hline Campleman, Sharan & $\begin{array}{l}004,022,038,040,074,109 \\
\quad 112,114 \\
115,117,118\end{array}$ \\
\hline Canning, Joshua & 076 \\
\hline Cantrell, Lee & 039 \\
\hline Cao, Dazhe & 130 \\
\hline Carpenter, Joe & 017,078 \\
\hline Carstairs, Shaun & 041 \\
\hline Castaneda, Jorge & 142 \\
\hline Castaneda, Maria & 009 \\
\hline Cerbini, Trevor & 143 \\
\hline Chang, Juliana & $073,098,101$ \\
\hline Charlton, Nathan & 189,190 \\
\hline Chen, Hsiang-Ling & 142 \\
\hline Cheng, Kai-Wen & 062 \\
\hline Chenoweth, James & 019,085 \\
\hline Chettat, Samy & 021 \\
\hline Choi, Jae-Hyek & 009 \\
\hline Clark, Richard & 092,123 \\
\hline Clemons, Joseph & 044 \\
\hline Cohen, Emily & 082 \\
\hline Cole, Ryan & 046,070 \\
\hline Collins, Samuel & 008 \\
\hline Colvin, Jonathan & 095 \\
\hline Coquyt, Dan & 034 \\
\hline Corbett, Bryan & 092,123 \\
\hline
\end{tabular}




\begin{tabular}{|c|c|c|c|}
\hline Correia, Matthew & $054,091,146$ & Friedman, Nathan & $003,039,057$ \\
\hline Crane, Peter & 012,029 & Froberg, Blake & 107 \\
\hline Crawford, Cindy & 011 & Furo, Hiroko & 188 \\
\hline Daigh, Arthur & 084,103 & Gaetani, Samantha & 047 \\
\hline De Olano, Jonathan & 089,136 & Galati, Bridget & 010 \\
\hline De Silva, Anjana & 001 & Galindo, Demi & 053 \\
\hline Deia, Mukta & 141 & Ganatra, Hammad & 066 \\
\hline DelBianco, John & 063 & Geib, Ann-Jeannette & 134 \\
\hline Delgado, Mucio Kit & 039,108 & Geller, Robert & 017 \\
\hline Deng, Jou-Fang & 142 & Germain, Michael & 081 \\
\hline Dernbach, Matthew & 094 & Gerona, Roy & 105,143 \\
\hline Desale, Sameer & 015 & Gill, Emily & 131 \\
\hline Deuster, Patricia & 011 & Giurca, Dan & 020 \\
\hline Devgun, Jason & 084 & Givens, Melissa & 011 \\
\hline Dewey, Rebecca & 032,034 & Glatstein, Miguel & 001 \\
\hline Dickenson, Carrie & 126 & Godsy, Grace & 010 \\
\hline Dimovska, Mirjana & 113,135 & Goertemoeller, Sheila & 095 \\
\hline Dion, Christopher & 121 & Goldfine, Charlotte & 109 \\
\hline Dizon, Mercedes & 093 & Goldman, Rose & 081 \\
\hline Dodson, Nicholas & 099,100 & Goodnough, Robert & 075 \\
\hline Dolcourt, Bram & 113,135 & Goodrich, Will & 070,189 \\
\hline Dominguez, Jacqueline & 093 & Gorodetsky, Rachel & 053 \\
\hline Dooley, Gregory & 002 & Graeme, Kimberlie & 142 \\
\hline Dowdell, Kimberly & 008 & Gragg, James & 045 \\
\hline Du Plessis, Arina & 031 & Graudins, Andis & 087 \\
\hline Dunavin, Adrienne & 141 & Griswold, Matthew & 046 \\
\hline Duvidovich, Svetlana & 098 & Gupta, Sudhir & 061,147 \\
\hline Earwood, Ryan & 106 & Guzman-Soto, Andres & 089 \\
\hline Eggleston, William & 006 & Hack, Jason & 006,090 \\
\hline Eisenberg, Jonathan & 055 & Hallett, Thao-phuong & 136 \\
\hline Eisenstat, Matthew & 122 & Hamer, Mark & 166 \\
\hline Ellison, Ross & 105,143 & Hamilton, Mikayla & 129 \\
\hline Ems, Raleigh & 110 & Hardika, Aanad & 001 \\
\hline Erickson, Timothy & 109 & Hardin, Jeremy & 106 \\
\hline Fahmy, Girgis & 025,033 & Harding, Stephen & 075 \\
\hline Farah, Rita & 013,017 & Harvey, Helen & 003 \\
\hline Farrell, Natalija & 090 & Haupts, Bradley & 049 \\
\hline Farrugia, Lynn & 046 & Heard, Kennon & 124 \\
\hline Faude, Sophia & 039,108 & Hein, Paul & 143 \\
\hline Ferguson, Ian & 048 & Heise, C & 016 \\
\hline Fialkoff, Sefira & 030,140 & Hendrickson, Audrey & 022 \\
\hline Fikse, Derek & $047,058,079,086,101$ & Hendrickson, Robert & $021,038,054,091$ \\
\hline Fil, Laura & $017,042,140$ & Herndon, Richard & 124 \\
\hline Filip, Ari & $048,067,084$ & Hibbard, Stephanie & 081 \\
\hline Finnegan, Jack & 059 & Hodgman, Michael & $020,026,028,140$ \\
\hline Fisher, Erik & 127 & Hoffman, Robert & 107 \\
\hline Fladby, Chris & 034 & Hogg-Bremer, Whitney & 036 \\
\hline Ford, Carrisa & 044 & Holian, Angela & 190 \\
\hline Fox, Erin & 023,024 & Holstege, Christopher & 008,085 \\
\hline Fredrickson, Caleb & 027,169 & Hon, Stephanie & 017 \\
\hline Frey, Aaron & 025,070 & Hopkinson, Andrew & 182 \\
\hline Friedman, Kyle & 002 & Horowitz, B Zane & 146 \\
\hline
\end{tabular}




\begin{tabular}{|c|c|}
\hline Horowitz, Keahi & 106 \\
\hline Howard, Cory & 017,028 \\
\hline Howshar, Jacob & 016 \\
\hline Hoyte, Christopher & 031 \\
\hline Hughes, Adrienne & 022,038 \\
\hline Hunter, Aimee & 011 \\
\hline Hurd, Yasmin & 003 \\
\hline Iglesias, Emma & 178 \\
\hline Illg, Zachary & 136 \\
\hline Irving, Jessica & 141 \\
\hline Irwin-Weyant, Morgan & 070 \\
\hline Isaacson, Andrew & 113 \\
\hline Isbister, Geoffrey & 001 \\
\hline Jacob, Jeena & 043 \\
\hline Jaiswal, Ashok & $035,061,147$ \\
\hline Jefri, Mohamed & 043,115 \\
\hline Jenniches, Daniel & 063 \\
\hline Ji, Michelle & 049 \\
\hline Joglekar, Mugdha & 001 \\
\hline Johnson, Amberly & 052 \\
\hline Johnson, Nicholas & 101 \\
\hline Johnson-Arbor, Kelly & 023,024 \\
\hline Joldersma, Kevin & 126 \\
\hline Kabala, Aimee & 103 \\
\hline Kadow, Elizabeth & 053 \\
\hline Kaiser, Sasha & 182 \\
\hline Kamanula, John & 130 \\
\hline Kamta, Jeff & 053 \\
\hline Kanojia, Rohit & 035,061 \\
\hline Kao, Louise & 084 \\
\hline Karim, Rehan & 104 \\
\hline Katz, Kenneth & 058,079 \\
\hline Kazzi, Ziad & $013,025,033,141$ \\
\hline Kebbi, Ola & 145 \\
\hline Kelly, Jeannie & 010 \\
\hline Kelly, Jessica & 024 \\
\hline Kerns, Abigail & 063 \\
\hline Khan, Ikhlas & 011 \\
\hline Kiat, Wenceslao & 093 \\
\hline Kiernan, Emily & 025,089 \\
\hline Kilaru, Austin & 039,108 \\
\hline Kim, Howard & 050 \\
\hline King, Andrew & 119,135 \\
\hline Kirschner, Ron & 034 \\
\hline Koons, Andrew & $047,058,079$ \\
\hline Kosnett, Michael & 002,025 \\
\hline Kosofsky, Michael & 056 \\
\hline Koura, Simran & 037,125 \\
\hline Kowalski, J & 120 \\
\hline Krotulski, Alex & $\begin{array}{l}004,038,040,109,112,114 \\
\quad 115\end{array}$ \\
\hline Kulik, Kelli & 075 \\
\hline
\end{tabular}

Kumar, Kapil 035

Labruzzo, Krista 121

Langner, Travis 066

Lank, Patrick 050

Laskey, Dayne 046

Lasoff, Daniel 057, 092

Lavon, Ophir $\quad 128$

Layer, Mark 025

Le, Danny 086

Le, Thuan 024

LeClair, Lorrie $\quad 131$

Lebin, Jacob 139

Lermer, Anné 031

Levine, Michael 004

Lewis, Brian 077

Lewis, Justin 019, 085

Lewis, Terese 034

$\mathrm{Li}$, Shao $\quad 114,117$

Lim, Derick 133

Lim, Timothy Reynold 093

Lima, Rafael 050

Lind, Nicholas 064

Linsey, Andrea $\quad 011$

Liss, David 010, 027, 048, 067, 084, 169

Liu, Joseph $\quad 088$

Logan, Barry $\quad 004,038,040,109,112,114$, 115

Longo, Lauren 018

Loo, George 014

Love, Jennifer $\quad 007,036,118$

Lowenstein, Margaret $\quad 039,108$

Lu, Jenny $\quad 096,159$

Ly, Binh 003

Lynch, Kara 102

Ma, Ming 002

Mack, Ethan 131

Maddry, Joseph 009

Mahonski, Sarah 082

Maker, Greg 138

Malley, Carin 076

Manini, Alex 004, 014, 038, 109, 112, 114, 115

003, 040

Marino, Ryan $\quad 089,110,111$

Marks, Carine 031

Marshall, Stacy 019

Martínez, Ana 178

Masdon, Joshua $\quad 037,125$

Mazer-Amirshahi, Maryann $\quad 015,023,024$

McCabe, Daniel 064, 102

McKay, Charles 141

Medina, Yiraima 059

Meehan, Timothy 132 
Meggs, William

Memon, Rmaah

Menon, Prem

Mete, Mihriye

Meyn, Alison

Mhango, Happy

Michienzi, Avery

Miklovic, Tyler

Miller, Andrew

Miller, Zachary

Milone, Michael

Minns, Alicia

Mithi, Vita

Moffett, Brady

Moghaddam, Hossein

Mohan, Sanjay

Mojica, Christianne

Monte, Andrew

Monuteaux, Michael

Morgan, Brent

Moss, Michael

Mostafazadeh, Babak

Mudan, Anita

Mullins, Matthew

Mullins, Michael

Murphy, Christine

Murphy, Lauren

Najari, Fares

Nelson, Lewis

Nemanich, Antonia

Neumann, Natalie

Ng, Patrick

Ngai, Ka Ming

Nguyen, Thu

Nguyen, HoanVu

Nguyen, Tuyet-Anh

Nguyen, Tuyet-anh

Nguyen, Vincent

Nillas, Andrea

Noble, Matthew

Nogar, Joshua

Norbeck, Kai

O'Connor, Ayrn

O'Donnell, Nicole

Oliff, Zachary

Olives, Travis

Osterhoudt, Kevin

Ozer, Vildan

Pacholski, Maurgerite

Palicherla, Anirudh

Pappas, Dominic
$006,037,125$
109
089
015

004, 022, 038, 040, 109, 112, 114

034

008, 070, 085

129

086

133

131

052, 069

032, 034, 130

075

093

082

093

182

065

017

055

004, 083

139

089

027, 048, 084, 169

134

007, 056

083

030, 036, 039, 108, 126

096

031

009

014

124

050

115

080

088

105,143

146

048, 051, 066, 071, 073, 097

098, 101

138

007

036

051,073

022,104

$018,024,131,133$

033

018

049

077
Paredes, R. Madelaine

Park, John

Parker-Cote, Jennifer

Parris, Mehruba

Pasol, Esteban

Patel, Khushbu

Patino-Calle, Daniel

Pei, Mitchell

Pepin, Lesley

Perrone, Jeanmarie

Phillips, Martha

Phillips, Todd

Philp, Allan

Phiri, Getrude

Piper, Lauren

Pizon, Anthony

Porter, Lauren

Posner, Jill

Puryear, Sarah

Quaidoo, Emmanuel

Radke, Joshua

Raffler, Rebecca

Rafizadeh, Ali

Rague, John

Rangan, Pooja

Rashid, Sonya

Repplinger, Daniel

Rianprakaisang, Tony

Richardson, Lynne

Riddle, Mark

Rigney, Elaine

Rizvi, Khizer

Roda, Gavriel

Rodriguez, Antonio

Rodriguez, Dylan

Rogers, Cynthia

Roper, Laura

Roper, Stephen

Ross, Bryan

Royalty, Alaina

Ruck, Bruce

Ruha, Michelle

Rushton, William

Ryan, Erin

Sahin, Aynur

Salahudeen, Shihana

Sarker, Abeed

Scanlon, Matthew

Schimmel, Jonathan

Schimpf, Mackenzie

Schmitz, Zachary

Schneir, Aaron
009

098

006

043, 080

093

024

046

145

050, 182

036, 039, 108, 116

137

130

063

034

134

076, 125

110, 111

024

068

139

064, 102

134

093

002

016

132

139

066, 099, 100

003

129

166

073

182

178

009

010

120

010

111

122

080

074

074

074

033

001

036, 116

076

014

045

157

003, 052, 069 
Schultz, Brian

Schwaner, Robert

Schwarz, Evan

Scott, Michael

Seltzer, Justin

Semple, Michael

Senart, Alicia

Seth, Devanshi

Shafer, Sarah

Shaker, Kerollos

Shastry, Siri

Shaw, Isaac

Shaw, Leslie

Shaw, Xian

Sheikh, Sophia

Sidlak, Alexander

Silberman, Zachary

Silver, Elizabeth

Simon, Mark

Simpson, Michael

Slane, Matthew

Smaltz, Lauren

Smith, Jesse

Smith, Silas

Smith, Stephen

Sorensen, Eric

Soto, Pelayia

Spadaro, Anthony

Spanier, Adam

Spyres, Meghan

Stariha, Jillian

Stashin, Amanda

Stein, Brandon

Stephan, Wendy

Stephani, Jennifer

Stephens, Robert

Stolbach, Andrew

Stott, Molly

Stout, Mackenzie

Stripp, Matthew

Su, Mark

Suen, Kyle

Surmaitis, Ryan

Sutariya, Ronak

Syed, Sameer

Tabin, Millo

Tak, Casey

Tancredi, Daniel

Tavornwattana, James

Tedford, Natalie

Temple, Courtney

Thakrar, Ashish
151

106

010, 067, 084, 114

060

003, 069, 092, 123

084

131

001

075

074, 105, 143

112

122

131

190

137

131,151

140

066

124

055, 060, 072, 113, 130

059

085

104

082

104

189

023, 024

036, 039, 108

138

007, 121, 138

044

058

044

018

021

067

126

137

052

063

082, 107, 157

089

086, 101

015

145

147

052

085

106

055

007, 022, 091

039, 108
Thompson, Arrianna

084

Thornton, Stephen

066

Tirado, Daniel

028

Titelbaum, Nicholas

078, 130, 136

Tormoehlen, Laura

103

Torres, Jose

106

Toxicology Investigators Consortium (ToxIC)

004, 013, 021, 022, 038, 040, 074, 099, 100, 109,112, 114, 115, 117, 124

Trautman, William 076

Tuaño, Bernardo Joaquin 093

Ubani, Chiemela 018

Uppal, Neelam 094

Vaidya, Rahul 113

Valencia, Vincent 093

Van Hoving, Daniël 031

Vargas-Torres, Carmen 003

Vidal, Kavey 003

Vohra, Varun 119, 135

Walsh, Steven $\quad 120$

Walter, Abraham 011

Wang, George 002

Warpinski, George 076

Watson, Christopher 055, 060, 065, 072, 113

Wax, Paul

004, 013, 022, 030, 038, 040, 074, 109

112, 114, 115 117, 118, 126, 138,140 ,

Weaver, Nicholas 055

Weber, Kelsey 084

Wei, Yudan 005

Weisman, Richard 018

Wermuth, Mary 068

Wheatley, Scott 058

White, Avian 037, 125

Whitledge, James $\quad 055,060,072,081,113$

Wiegand, Timothy 012, 029, 053, 094, 140, 166

Wiener, Sage 143

Wijeyesakere, Pioma $\quad 087$

Willis, James 143

Wilson, Bryan 064, 102

Winkler, Garret 052

Wolfe, Jordan 090

Wong, Edric 099, 100

Woolf, Alan 081

Wu, Alan 157

Wu, Ming-Ling 142

Yakey, Brandtly 113

Yang, Chen-Chang 142

Yanta, Joseph 076 
Yarra, Silpa

157

Yin, Shan

095

Zabel, Andrew

138

Zamani, Nasim

093

Zdradzinski, Michael

089

Zhu, Jianmin

005

Publisher's Note Springer Nature remains neutral with regard to jurisdictional claims in published maps and institutional affiliations. 